SFB

Monitoring stationarity and 823 cointegration

Martin Wagner, Dominik Wied

Nr. 23/2014
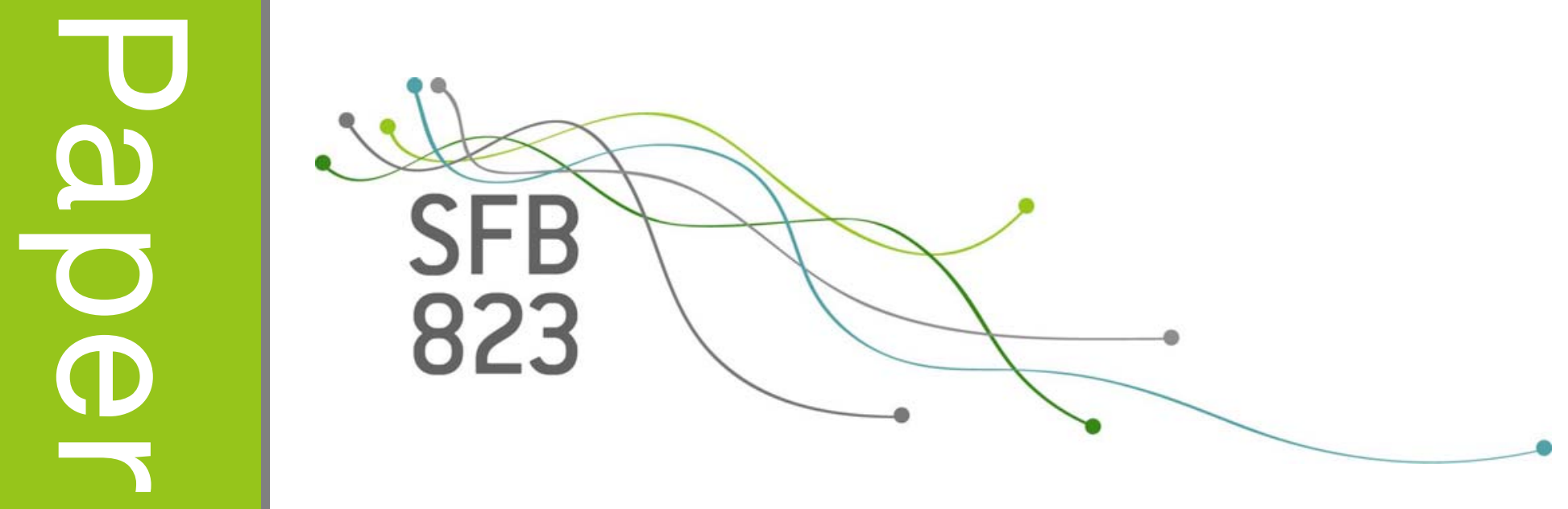



\title{
MONITORING STATIONARITY AND COINTEGRATION
}

\author{
Martin Wagner \& Dominik Wied* \\ Faculty of Statistics, Technical University Dortmund \\ Institute for Advanced Studies, Vienna \\ \& \\ Faculty of Statistics, Technical University Dortmund
}

\begin{abstract}
We propose a monitoring procedure to detect a structural change from stationary to integrated behavior. When the procedure is applied to the residuals of a relationship between integrated series it thus monitors a structural change from a cointegrating relationship to a spurious relationship. The cointegration monitoring procedure is based on residuals from modified least squares estimation, using either Fully Modified, Dynamic or Integrated Modified OLS. The procedure is inspired by Chu et al. (1996) in that it is based on parameter estimation on a pre-break "calibration" period only rather than being based on sequential estimation over the full sample. We investigate the asymptotic behavior of the procedures under the null, for (fixed and local) alternatives and in case of parameter changes. We also study the finite sample performance via simulations. An application to credit default swap spreads illustrates the potential usefulness of the procedure.
\end{abstract}

Keywords: Cointegration, Monitoring, Stationarity, Structural Change, Unit Roots

JEL Codes: C22, C32, C52

*Technical University Dortmund, Faculty of Statistics, Vogelpothsweg 87, D-44227 Dortmund, Germany. E-Mail: mwagner@statistik.tu-dortmund.de, Phone: +49 2317553174 (M. Wagner). E-Mail: wied@statistik.tu-dortmund.de, Phone: +49 2317555419 (D. Wied). Financial support from Deutsche Forschungsgemeinschaft via the Collaborative Research Center 823: Statistical Modelling of Nonlinear Dynamic Processes (Projects A1, A3 and A4) is gratefully acknowledged. The first author furthermore acknowledges financial support from the Jubiläumfonds of the Oesterreichische Nationalbank (Grant No. 15334). We are grateful to Philipp Aschersleben for excellent research assistance. The usual disclaimer applies. 


\section{INTRODUCTION}

It is common practice in time series econometrics to investigate the stationarity, unit root and cointegration properties of time series and a plethora of tests for stationarity, unit roots and cointegration is available. In relation to this practice, however, it may well be reasonable to investigate the question whether the stationarity or cointegration behavior of time series changes over time. In particular, a time series may change its behavior from stationarity, or being $\mathrm{I}(0)$, to being integrated and a cointegrating relationship between several time series may break down and turn into a spurious relationship. ${ }^{1}$ Examples where one may be concerned about this type of structural change include deviations from purchasing power parity after a period of international economic stability or nonstationarity of credit default swap (CDS) spreads after the onset of a financial crisis. $^{2}$

Our monitoring procedure is inspired by the monitoring procedure for linear regression models of Chu et al. (1996) in that parameter estimation, for estimating trend and when monitoring cointegration - slope parameters, is based solely on a "calibration" period at the beginning of the sample that is known or assumed to be free of structural change. $^{3}$ Based on the parameter estimates, computed using only calibration period data, the detrended observations (or the residuals of a cointegrating relationship) are the key ingredient for the monitoring procedure. The monitoring procedure is based on sequentially computing the differences of scaled partial sums of squared residuals over the growing monitoring period and the calibration period. ${ }^{4}$ The detection time, defined in the following section, serves as an immediately available estimate of the break-point. In order to obtain nuisance parameter free limiting distributions of the test statistics

\footnotetext{
${ }^{1}$ As discussed below, the approach also allows to monitor whether the coefficients of a cointegrating relationship change over time.

${ }^{2}$ Related issues are analyzed in tests for so-called asset price bubbles, see e.g. Phillips et al. (2011), where a bubble is associated with a structural change towards explosive behavior. Our approach can be applied in this context as well, as discussed later.

${ }^{3}$ This approach to monitoring, based on estimation in a pre-break sample period only, has been extended to the multivariate linear regression case by Groen et al. (2013) and has been applied to monitor changes in the correlation structure by Wied and Galeano (2013).

${ }^{4}$ This, of course, immediately implies consistency of the procedure against any "more explosive" alternative, like higher order integration or explosive behavior.
} 
when applying the principle to monitor cointegrating relationships, parameter estimation on the calibration sample is performed using any of the available modified least squares estimators that lead to nuisance parameter free limiting distributions of the parameters of the cointegrating relationship. In particular we consider here Fully Modified OLS (FM-OLS) of Phillips and Hansen (1990), Dynamic OLS (D-OLS) of Saikkonen (1991), Phillips and Loretan (1993) and Stock and Watson (1993), and Integrated Modified OLS (IM-OLS) of Vogelsang and Wagner (2014).

The asymptotic properties of the monitoring procedures are derived under both the null as well as under (fixed and local) alternatives and for the case of breaks in trend parameters. In case of cointegration monitoring we additionally consider the asymptotic behavior of the monitoring procedure in case of breaks in the slope parameters. Based on the asymptotic results, the performance of the proposed methods is investigated by means of local asymptotic power analysis. Furthermore, finite sample simulations are performed to consider empirical size and power for a variety of scenarios. Again, in addition to studying the power against the alternative of integrated behavior we also assess the performance in case of parameter changes in the trend and/or slope coefficients. We also assess the estimated detection times in the finite sample simulations.

We briefly illustrate our monitoring procedure using daily CDS spreads series for Austria, Cyprus, France and Germany over the period April 3, 2009 to August 1, 2012. The null hypothesis of no structural change from stationary to integrated behavior is rejected for all countries and all five considered maturities. For most of the series the estimated break date is found to be in summer or fall 2010, i.e. already almost a year before the CDS spreads series started to be dramatically high starting in summer 2011.

The paper is organized as follows: Section 2 develops the stationarity monitoring procedure and studies its asymptotic properties under the null, for fixed and local alternatives and in case of trend breaks. In Section 3 the approach is extended to monitor cointegration and the asymptotic properties of the monitoring procedure are discussed for the above mentioned three estimation procedures for cointegrating relationships. In addition 
we discuss here also the properties in case of structural change in the slope parameters. Section 4 provides some simulation results investigating the finite sample properties of the proposed monitoring procedures and Section 5 contains a brief illustration of the monitoring procedure using CDS spreads data for four European countries. Finally, Section 6 summarizes and concludes. All proofs are deferred to Appendix A and Appendix B provides tables with critical values. Supplementary material containing additional simulation and empirical results as well as additional critical values is available upon request. Furthermore, also code that implements the discussed methods is available upon request.

\section{Model And Assumptions for Monitoring Stationarity}

The starting point of our considerations is to monitor (trend-)stationarity of

$$
y_{t}=D_{t}^{\prime} \theta_{D}+u_{t}
$$

for which a sample of observations for $t=1, \ldots, T$ is available and where $D_{t} \in \mathbb{R}^{p}$ is a deterministic trend function, with coefficients $\theta_{D} \in \mathbb{R}^{p}$, for which the following assumption is made:

Assumption 1. There exists a sequence of $p \times p$ scaling matrices $G_{D}$ and a $p$-dimensional vector of functions $D(z)$, with $0<\int_{0}^{s} D(z) D(z)^{\prime} d z<\infty$ for $0 \leq s \leq 1$, such that for $0 \leq s \leq 1$

$$
\lim _{T \rightarrow \infty} \sqrt{T} G_{D}^{-1} D_{[s T]} \rightarrow D(s)
$$

with $[s T]$ denoting the integer part of $s T$.

If e.g. $D_{t}=\left(1, t, t^{2}, \ldots, t^{p-1}\right)^{\prime}$, then $G_{D}=\operatorname{diag}\left(T^{1 / 2}, T^{3 / 2}, T^{5 / 2}, \ldots, T^{p-1 / 2}\right)$ and $D(z)=$ $\left(1, z, z^{2}, \ldots, z^{p-1}\right)^{\prime}$.

Under the null hypothesis of trend stationarity we posit the assumption that the process $\left\{u_{t}\right\}_{t \in \mathbb{Z}}$ fulfills a functional central limit theorem (FCLT): 
Assumption 2. The stationary process $\left\{u_{t}\right\}_{t \in \mathbb{Z}}$ fulfills

$$
\frac{1}{\sqrt{T}} \sum_{t=1}^{[s T]} u_{t} \Rightarrow \omega W(s)
$$

where $W(s)$ denotes standard Brownian motion and $0<\omega^{2}<\infty$ is the long-run variance of $\left\{u_{t}\right\}_{t \in \mathbb{Z}}$,

$$
\omega^{2}:=\sum_{j=-\infty}^{\infty} \mathbb{E}\left(u_{t} u_{t-j}\right)
$$

These two assumptions basically reflect the need for consistent OLS detrending and longrun variance estimation (see also Footnote 2) and are extended in the following section to allow for consistent parameter estimation in cointegrating regressions by any of the mentioned modified least squares techniques. Any set of assumptions that leads to these convergence results can be used.

In this paper we refer to a stochastic process fulfilling Assumption 2 as an $\mathrm{I}(0)$ process. Requiring $\omega^{2}>0$ excludes over-differenced processes, e.g. $u_{t}=\varepsilon_{t}-\varepsilon_{t-1}, t \in \mathbb{Z}$ for some white noise process $\left\{\varepsilon_{t}\right\}_{t \in \mathbb{Z}}$ has long-run variance equal to 0 .

Remark 1. Note that we do not need stationarity in our $\mathrm{I}(0)$ definition, but only the mentioned functional central limit result (3), with $\omega^{2}=\lim _{T \rightarrow \infty} \mathbb{E}\left(\frac{1}{T} \sum_{t=1}^{T} u_{t}\right)^{2}$. All results hold without the extra requirement of stationarity in Assumption 2. We include stationarity in the assumption, since we later consider monitoring of cointegration, i.e. linear combinations of series that are stationary (to stick to the usual definition of cointegration).

Under the alternative we consider the situation that there exists some time point $[r T]$ such that the process behaves like an $\mathrm{I}(1)$ process from $[r T]+1$ onwards. Thus, under the alternative Assumption 2 is violated from $[r T]+1$ onwards in a specific way. For our paper we define a process $\left\{x_{t}\right\}_{t \in \mathbb{Z}}$ to be an I(1) process, in accordance with our $\mathrm{I}(0)$ 
definition, if

$$
\frac{1}{\sqrt{T}} x_{[s T]} \Rightarrow \omega W(s)
$$

for $0 \leq s \leq 1$, some $0<\omega^{2}<\infty$ and where $W(s)$ denotes again standard Brownian motion. It is clear that the (partial) sum process of an $\mathrm{I}(0)$ process is an $\mathrm{I}(1)$ process. Thus, our null and alternative hypotheses are in formal terms given by

$$
\begin{aligned}
H_{0}: \frac{1}{\sqrt{T}} \sum_{t=1}^{[s T]} u_{t} \Rightarrow & \omega W(s), \text { for all } 0 \leq s \leq 1 \\
H_{1}: \frac{1}{\sqrt{T}} \sum_{t=[r T]+1}^{[s T]} u_{t}= & O_{p}\left(T^{1 / 2}\right) \text { and } \frac{1}{\sqrt{T}} \sum_{t=[r T]+1}^{[s T]} u_{t} \neq o_{p}\left(T^{1 / 2}\right) \\
& \text { for some } 1>r \geq m>0 \text { and for all } s>r
\end{aligned}
$$

The above formulation is to be understood in the sense that also under the alternative the process $\left\{u_{t}\right\}_{t \in \mathbb{Z}}$ fulfills Assumption 2 up to $[r T] \geq[m T]$. Note that we want to detect a change from $\mathrm{I}(0)$ to $\mathrm{I}(1)$ behavior under the alternative that occurs at time point $[r T]$ with $m \leq r<1$, i.e. a change that occurs only after some pre-break sample fraction of length $[m T]$ with $0<m<1$.

As we shall see below, we need such a pre-break sample fraction $m$ in particular in order to consistently estimate several quantities required to obtain a null limiting distribution of our detector that is a function only of the included deterministic components and standard Brownian motions and for which thus critical values can be simulated. These quantities include, depending upon situation considered, the long-run variance $\omega^{2}$, the trend parameters $\theta_{D}$ and in the following section, dealing with cointegration, also slope parameters $\theta_{X}$ corresponding to the $\mathrm{I}(1)$ regressors in the monitored cointegrating relationship.

Remark 2. As mentioned in the introduction, our approach is inspired by Chu et al. (1996), albeit we frame the problem slightly differently. As is standard in the unit root 
and cointegration literature we map the (full set of) observations $1, \ldots, T$ in the interval $[0,1]$, as $T \rightarrow \infty$, and thus in the limit our pre-break estimation period corresponds to the interval $[0, m]$, as we use observations $1, \ldots,[m T]$ for parameter estimation. Chu et al. (1996) consider as their "historical" period observations $1, \ldots, m$. In their asymptotic analysis $m$, with $m \rightarrow \infty$, is considered a fraction of the overall sample size $T=\lambda m$ with $\lambda>1$. Thus, the observations $1, \ldots, m$ are mapped into the interval $[0,1]$ and the whole set of observations into $[0, \lambda]$.

Considering for the moment $u_{t}, t=1, \ldots, T$ observed and $\omega^{2}$ known suffices to discuss the approach of the paper. In this idealized case the detector for $\left\{u_{t}\right\}_{t \in \mathbb{Z}}$ is given by

$$
H^{m}(s):=\frac{1}{\omega^{2}}\left(\frac{1}{T} \sum_{i=[m T]+1}^{[s T]}\left(\frac{1}{\sqrt{T}} S_{i}\right)^{2}-\frac{1}{T} \sum_{i=1}^{[m T]}\left(\frac{1}{\sqrt{T}} S_{i}\right)^{2}\right)
$$

for $0<m \leq s \leq 1$ and with $S_{i}=\sum_{t=1}^{i} u_{t}$ denoting the partial sums of $u_{t}$.

Under Assumption 2 it holds under the null hypothesis of no structural change that

$$
H^{m}(s) \Rightarrow \mathcal{H}^{m}(s):=\left(\int_{m}^{s} W(z)^{2} d z-\int_{0}^{m} W(z)^{2} d z\right)
$$

whereas the detector will diverge under the alternative (see the discussion below for details). The detector is inspired by the KPSS stationarity test of Kwiatkowski et al. (1992), with the (idealized) test statistic given by $\frac{1}{\omega^{2}}\left(\frac{1}{T} \sum_{i=1}^{T}\left(\frac{1}{\sqrt{T}} S_{i}\right)^{2}\right)$, which converges to $\int_{0}^{1} W(z)^{2} d z$ under the null of stationarity. In case of $\mathrm{I}(1)$ behavior the scaled sum diverges and our detector exploits these convergence rate differences by comparing the convergent pre-break quantity with the potentially diverging post-break quantity. ${ }^{5}$

A related procedure is provided by Steland (2007), who bases his monitoring procedure on sequential kernel-weighted variance ratios, i.e. his detector to monitor a change from $\mathrm{I}(0)$ to I(1) behavior is, using our notation and known $\omega^{2}$, given by $\tilde{U}_{T}(s)=\frac{1}{\omega^{2} T} \sum_{i=1}^{[s T]} S_{i}^{2} K_{h}(i-$

\footnotetext{
${ }^{5}$ Let us note already here that in the cointegration monitoring situation our detector is similarly based on the cointegration test statistic of Shin (1994) that extends the KPSS test from a stationarity to a cointegration test.
} 
$[s T]$ ) for some kernel function $K_{h}(\cdot)$. Steland (2007) also considers monitoring a change from $I(1)$ to $I(0)$ behavior (clearly, with a differently scaled detector than the one given above). ${ }^{6}$ Steland and Weidauer (2013) extend this approach to regressions with integrated regressors that are, however, assumed to be strictly exogenous or even independent of the errors. Also in this paper, as in Steland (2007), the null hypothesis is that of I(1) errors against the alternative of $\mathrm{I}(0)$ errors after the break. In this paper we consider, in the following section, monitoring the null of cointegration ( $\mathrm{I}(0)$ errors), allowing for endogenous regressors, against the alternative of a spurious regression (I(1) errors).

We define the detection time $\tau_{m}\left(H^{m}, g, c\right)$, often only written as $\tau_{m}$ if the context is clear, as

$$
\tau_{m}:=\min _{s:[m T]+1 \leq[s T] \leq T}\left\{\left|\frac{H^{m}(s)}{g(s)}\right|>c\right\}
$$

i.e. the null hypothesis is declared rejected when the standardized detector, $\frac{H^{m}(s)}{g(s)}$, exceeds a critical value $c$ in absolute value for the first time. In case that $\left|\frac{H^{m}(s)}{g(s)}\right| \leq c$ for all $m \leq s \leq 1$ we write $\tau_{m}=\infty$. Thus, a finite value of $\tau_{m}$ indicates a rejection of the null and at the same time gives information about the potential break point.

The properties of such a monitoring procedure hinge, by construction, upon the threshold function $g(s)$ and the constant $c$, which itself depends upon the function $g$. For simplicity we only consider weighting functions that are continuous, bounded and positive throughout this paper, compare Assumption 3.6 of Aue et al., 2012. Note that Chu et al. (1996) consider more general weighting functions. Weighting function and critical value have to

\footnotetext{
${ }^{6}$ Note that Steland (2007) does not consider deterministic components. This restriction is relaxed to a certain extent in Steland (2008), where he considers polynomial trends. This situation is also considered in Qi et al. (2013). Chen et al. (2010) modify the approach of Steland and also use a "calibration" period at the beginning of the sample for which it is known whether the series is $\mathrm{I}(0)$ or $\mathrm{I}(1)$. Chen et al. (2012) use the bootstrap to detect multiple changes of persistence. Another related procedure is discussed in Kim (2000), who considers the properly scaled ratio of sums of squared partial sums of residuals before and after the hypothesized break.
} 
be chosen in order to ensure that under the null hypothesis

$$
\begin{aligned}
\lim _{T \rightarrow \infty} \mathbb{P}\left(\tau_{m}<\infty\right) & =\lim _{T \rightarrow \infty} \mathbb{P}\left(\min _{s:[m T]+1 \leq[s T] \leq T}\left\{\left|\frac{H^{m}(s)}{g(s)}\right|>c\right\}<\infty\right) \\
& =\lim _{T \rightarrow \infty} \mathbb{P}\left(\sup _{s:[m T]+1 \leq[s T] \leq T}\left|\frac{H^{m}(s)}{g(s)}\right|>c\right) \\
& =\mathbb{P}\left(\sup _{m \leq s \leq 1}\left|\frac{\mathcal{H}^{m}(s)}{g(s)}\right|>c\right)=\alpha,
\end{aligned}
$$

with $\alpha$ denoting the chosen significance level. Deriving such a result can be based on a functional central limit theorem for $H^{m}(s)$, since we consider only continuous $g(s)$. The choice of $g(s)$ is in the words of Chu et al. (1996), compare p. 1052, "often dictated by mathematical convenience rather than optimality, since crossing probabilities for an arbitrary boundary are analytically intractable in general." The problem is typically transformed into delivering simple stopping times. This is due to the fact, already used in (11), that the event $\left\{\inf _{m \leq s \leq 1}\left\{\left|\mathcal{H}^{m}(s) / g(s)\right|>c\right\} \neq \infty\right\}$ is equal to the event $\left\{\sup _{m \leq s \leq 1}\left\{\left|\mathcal{H}^{m}(s) / g(s)\right|\right\}>c\right\}$. Therefore, the sup-functional in the context of testing can be considered as the natural equivalent to the stopping time based on the inf-functional in the context of monitoring. This is also the approach pursued in Chu et al. (1996) for parameter change in linear regression models. Clearly, the choice of the weighting function impacts the performance of the monitoring procedure. The choice of the weighting function has to combine two opposing goals of a monitoring procedure: (a) small size distortions and (b) small delays, i.e. detection soon after the break. The discussion in Section 3 of Chu et al. (1996) makes clear that it will in general be impossible to derive analytically tractable optimal weighting functions, e.g. with respect to minimal expected delay whilst asymptotically controlling size. ${ }^{7}$

With the starting point in most applications being the observed time series $y_{t}, t=$ $1, \ldots, T$ rather than $u_{t}, t=1, \ldots, T$, partial sums of residuals are the input in the

\footnotetext{
${ }^{7}$ Aue et al. (2009) derive the limiting distribution of the delay time for a one-time parameter change in a linear regression model with stationary regressors for a simple class of weighting functions depending on a single tuning parameter. To the best of the authors' knowledge, no results of this kind are available in a unit root or cointegration setting.
} 
monitoring procedure. The residuals $\hat{u}_{t, m}$ are given by detrended $y_{t}$, with the trend parameters $\theta_{D}$ estimated from the pre-break sample $t=1, \ldots,[m T]$. Thus,

$$
\begin{aligned}
\hat{u}_{t, m} & :=y_{t}-D_{t}^{\prime} \hat{\theta}_{D, m} \\
& =u_{t}-D_{t}^{\prime}\left(\hat{\theta}_{D, m}-\theta_{D}\right) \\
& =u_{t}-D_{t}^{\prime}\left(\sum_{i=1}^{[m T]} D_{i} D_{i}^{\prime}\right) \sum_{i=1}^{-1} D_{i} u_{i} .
\end{aligned}
$$

Under Assumptions 1 and 2 it follows immediately that

$$
\frac{1}{\sqrt{T}} \sum_{t=1}^{[s T]} \hat{u}_{t, m}=\frac{1}{\sqrt{T}} \widehat{S}_{[s T]} \Rightarrow \omega \widehat{W}_{m}(s)
$$

with $\widehat{S}_{i}=\sum_{t=1}^{i} \hat{u}_{t, m}$ and $\widehat{W}_{m}(s):=W(s)-\int_{0}^{s} D(z)^{\prime} d z\left(\int_{0}^{m} D(z) D(z)^{\prime} d z\right)^{-1} \int_{0}^{m} D(z) d W(z)$. Given the FCLT (13) for the partial sum of the detrended observations and the continuous mapping theorem, the asymptotic behavior of the detector based on $\hat{u}_{t, m}, \widehat{H}^{m}(s)$ say, under the null hypothesis can be established.

Lemma 1. Let the data be generated by (1) with Assumptions 1 and 2 in place and let $\hat{\omega}_{m}^{2}$ denote a consistent long-run variance estimator based on $\hat{u}_{t, m}$, for $t=1, \ldots,[m T]$. Then it holds under the null hypothesis for $T \rightarrow \infty$ and $m \leq s \leq 1$ that

$$
\begin{aligned}
\widehat{H}^{m}(s) & :=\frac{1}{\hat{\omega}_{m}^{2}}\left(\frac{1}{T} \sum_{i=[m T]+1}^{[s T]}\left(\frac{1}{\sqrt{T}} \widehat{S}_{i}\right)^{2}-\frac{1}{T} \sum_{i=1}^{[m T]}\left(\frac{1}{\sqrt{T}} \widehat{S}_{i}\right)^{2}\right) \\
& \Rightarrow \int_{m}^{s} \widehat{W}_{m}^{2}(z) d z-\int_{0}^{m} \widehat{W}_{m}^{2}(z) d z=: \widehat{\mathcal{H}}^{m}(s) .
\end{aligned}
$$

In order to show consistency of the detector later, it is of key importance here that all parameters, i.e. the trend slopes $\theta_{D}$ and the long-run variance $\omega^{2}$, are estimated only from the pre-break sample up to $[m T]$. Consistent long-run variance estimation is a well studied problem in the econometrics literature and has been established for a variety of primitive or high level assumptions. For simplicity in this paper we merely assume that 
the sufficient assumptions on $\left\{u_{t}\right\}_{t \in \mathbb{Z}}$, the kernel function and bandwidth given in Jansson (2002) are fulfilled. ${ }^{8}$

Under the stated assumptions it can be shown that under the null hypothesis, for given weighting function $g(s)$, there exist critical values $c=c(\alpha, g)$, which depend not only on $\alpha$ but also on the weighting function $g$, such that the detection time is finite with probability equal to the pre-specified level $\alpha$.

Proposition 1. Let the data be generated by (1) with Assumptions 1 and 2 in place and let $\hat{\omega}_{m}^{2}$ denote a consistent long-run variance estimator. Then, under the null hypothesis it holds that for any given $0<\alpha<1$ and any continuous function $g$ such that $0<g(s)<\infty$ there exists a $0<c=c(\alpha, g)<\infty$, such that

$$
\lim _{T \rightarrow \infty} \mathbb{P}\left(\tau_{m}\left(\widehat{H}^{m}, g, c(\alpha, g)\right)<\infty\right)=\alpha
$$

Given the behavior under the null hypothesis the next result shows that the monitoring procedure is consistent against both fixed and local alternatives, defined precisely below. As fixed alternative we consider the case that $\left\{u_{t}\right\}_{t \in \mathbb{Z}}$ changes behavior from $\mathrm{I}(0)$ to $\mathrm{I}(1)$ at some point after $[m T]$, i.e. that $H_{1}$ as given above holds. To understand the properties of our procedure in more detail we also consider local alternatives of the following form (inspired by Cappuccio and Lubian, 2005). There exists an $r$, with $m \leq r<1$ such that for all $t \leq[r T]$ we have $u_{t}=u_{t}^{0}$, while for all $t>[r T]$ it holds that

$$
u_{t}=u_{t}^{0}+\frac{\delta}{T} \sum_{i=[r T]+1}^{t} \xi_{i}
$$

with $\left\{u_{t}^{0}\right\}_{t \in \mathbb{Z}}$ and $\left\{\xi_{t}\right\}_{t \in \mathbb{Z}}$ independent processes both fulfilling Assumption 2, with longrun variances $\omega^{2}$ and $\omega_{\xi}^{2}$, and $\delta>0$. I.e. under the considered local alternatives the

\footnotetext{
${ }^{8}$ To be precise this means to specify more detailed assumptions on $\left\{u_{t}\right\}_{t \in \mathbb{Z}}$ than just the FCLT formulated in Assumption 2, compare Assumptions A1 and A2 in Jansson (2002). Let us note again, that any other set of assumptions that allows for consistent long-run variance estimation also would serve our purposes. In relation to Remark 1 it has to be noted that without the stationarity assumption one has to resort to other conditions, since Jansson (2002) considers stationary processes.
} 
process $\left\{u_{t}\right\}_{t \in \mathbb{Z}}$ is, from time point $[r T]+1$ onwards, the sum of an $\mathrm{I}(0)$ process and an independent I(1) process divided by the sample size. The local alternatives imply that the properly scaled partial sums of $\hat{u}_{t, m}$, i.e. $\widehat{S}_{t}$, converge to the following expression (for details see the proofs in Appendix A):

$$
\frac{1}{\sqrt{T}} \widehat{S}_{[s T]} \Rightarrow \omega \widehat{W}_{m}(s)+\delta \omega_{\xi} \int_{r}^{s}\left(W_{\xi}(z)-W_{\xi}(r)\right) d z
$$

where integrals (and sums) with the lower boundary larger than the upper are defined to be equal to 0 . Here $W_{\xi}(s)$ is a standard Brownian motion independent of $W(s)$.

\section{Proposition 2. (Consistency and Local Asymptotic Power)}

Let the data be generated by (1) with Assumption 1 in place and with $\left\{u_{t}\right\}_{t \in \mathbb{Z}}$ fulfilling Assumption 2 until $[r T]$, with $m \leq r<1$. Furthermore, let $\hat{\omega}_{m}^{2}$ again be a consistent long-run variance estimator and $g$ continuous with $0<g(s)<\infty$.

(a) Let $\left\{u_{t}\right\}_{t \in \mathbb{Z}}$ be an I(1) process (as specified in $H_{1}$ ) from $[r T]+1$ onwards. Then the monitoring procedure is consistent, i.e. for any $c>0$ it holds that

$$
\lim _{T \rightarrow \infty} \mathbb{P}\left(\tau_{m}\left(\widehat{H}^{m}, g, c\right)<\infty\right)=1
$$

(b) Let $\left\{u_{t}\right\}_{t \in \mathbb{Z}}$ be as specified in (17) from $[r T]+1$ onwards. Then the monitoring procedure has non-trivial local power. That means that for any $1-\alpha \geq \epsilon>0$ and the $0<c=c(\alpha, g)<\infty$ from Proposition 1 there exists $a 0<\delta=\delta(c, g)<\infty$ such that

$$
\lim _{T \rightarrow \infty} \mathbb{P}\left(\tau_{m}\left(\widehat{H}^{m}, g, c(\alpha, g)\right)<\infty\right) \geq 1-\epsilon
$$

The result underlying part (b) stems from the convergence result for $\widehat{H}^{m}(s)$ under the 
considered local alternatives. For $1 \geq s>r \geq m$ it holds that:

$$
\begin{aligned}
\widehat{H}^{m}(s) \Rightarrow & \widehat{\mathcal{H}}^{m}(s)+2 \delta \frac{\omega_{\xi}}{\omega} \int_{r}^{s} \widehat{W}_{m}(z)\left(\int_{r}^{z}\left(W_{\xi}(g)-W_{\xi}(r)\right) d g\right) d z+ \\
& +\delta^{2}\left(\frac{\omega_{\xi}}{\omega}\right)^{2} \int_{r}^{s}\left(\int_{r}^{z}\left(W_{\xi}(g)-W_{\xi}(r)\right) d g\right)^{2} d z
\end{aligned}
$$

This result shows that the magnitude of the additional terms depends, in addition to $\delta$, upon the "signal-to-noise" ratio $\omega_{\xi} / \omega$. As expected, $\omega$ enters with negative powers, i.e. a larger error variance decreases local asymptotic power and similarly a larger variance of the additional I(1) component increases local asymptotic power. ${ }^{9}$

Remark 3. It is clear and immediate from an inspection of the proof that the procedure is consistent not only against the $\mathrm{I}(1)$ alternative but also against the alternative of near-integrated processes, compare Phillips (1987). A near-integrated process is given by $u_{t, T}=\left(1-\frac{d}{T}\right) u_{t-1, T}+\nu_{t}$, with $d>0$ and $\left\{\nu_{t}\right\}_{t \in \mathbb{Z}}$ a stationary process with finite and positive long-run variance $\omega^{2}$. Clearly, in case $d=0$ we are back to the standard I(1) alternative. Consistency against near-integrated alternatives follows from the functional central limit theorem for near-integrated processes, i.e. $\frac{1}{\sqrt{T}} u_{[r T], T} \Rightarrow \omega V_{d}(r)$, with $V_{d}(r)=$ $\int_{0}^{r} e^{-d(r-s)} d W(s)$, which implies that near-integrated alternatives fulfill $H_{1}$.

The above results do not pin down the threshold function $g(s)$, which could be specified in many ways. Given that optimality results are not available and potentially not possible to obtain, a natural and simple candidate is to choose $g$ in relation to $\mathbb{E}\left(\widehat{\mathcal{H}}^{m}\right)$ to standardize the first moment of the detector's distribution under the null. In the special case of an intercept only $\left(D_{t}=1\right)$, we obtain

$$
\widehat{W}_{m}(s)=W(s)-\frac{s}{m} \int_{0}^{m} d W(z)=W(s)-\frac{s}{m} W(m) .
$$

\footnotetext{
${ }^{9}$ In the local asymptotic power simulations below we set the signal-to-noise ratio without loss of generality equal to one, since local asymptotic power depends only upon the product of $\delta$ and the signalto-noise ratio. Also note that it is sufficient to consider $\left\{u_{t}^{0}\right\}_{t \in \mathbb{Z}}$ and $\left\{\xi_{t}\right\}_{t \in \mathbb{Z}}$ independent, as asymptotic independence between the two components can always be achieved by redefining the two quantities correspondingly after "orthogonalization".
} 
Then, with the well-known covariance structure of a standard Brownian motion one obtains

$$
\mathbb{E}\left(\widehat{W}_{m}^{2}(s)\right)=s-\frac{2 s}{m} \min (s, m)+\frac{s^{2}}{m}
$$

from which, by changing the sequence of integration (Fubini), it follows that

$$
\mathbb{E}\left(\widehat{\mathcal{H}}^{m}(s)\right)=\int_{m}^{s} \mathbb{E}\left(\widehat{W}_{m}^{2}(z)\right) d z-\int_{0}^{m} \mathbb{E}\left(\widehat{W}_{m}^{2}(z)\right) d z=-\frac{1}{2} s^{2}+\frac{1}{3} \frac{s^{3}}{m}
$$

Thus, the order of the expected value is $s^{3}$, which motivates our choice $g(s)=s^{3}$ to essentially standardize the expected value of the detector. In case that the deterministic component consists of intercept and linear trend $\left(D_{t}=(1, t)\right)$, similar calculations lead to the order being $s^{5}$. Thus, in the linear trend case we consider $g(s)=s^{5}$ in the simulations and application below.

For given or chosen weighting function, critical values for the test procedure can be simulated by approximating the functionals of Brownian motions by the corresponding functions of random walks of length 1,000 generated from i.i.d. standard normal random variables. The available critical values are based on 1,000,000 replications. The critical values depend upon $0<m<1$ and the specification of the deterministic component. Detailed critical values for a grid of $m$-values ranging from 0.1 to 0.9 with mesh 0.01 are contained in Appendix B, in Table 4, for $D_{t}=1$ with $g(s)=s^{3}$, and in Table 5 , for $D_{t}=(1, t)^{\prime}$ with $g(s)=s^{5}$.

The monitoring procedure can also be used to detect breaks in the trend parameters $\theta_{D}$ that occur after $[m T]$, given that the trend parameters are also estimated only using the observations up to $[m T]$. Again we consider fixed and local alternatives. ${ }^{10}$ In the present context local alternatives are not described by a scalar parameter, but by $\Delta_{\theta} \in \mathbb{R}^{p}$ and the appropriate local alternatives are given by $G_{D}^{-1 \prime} \Delta_{\theta}$, reflecting the different rates of

\footnotetext{
${ }^{10}$ The usage of the word alternative is sloppy here, since it is now not the stochastic component that leads to divergence of the detector. We are confident that this will not lead to any confusion.
} 
convergence of the trend parameters. E.g. for the constant term, the rate is the usual $\frac{1}{\sqrt{T}}$, whereas for the linear trend coefficient the rate is $T^{-3 / 2}$.

\section{Proposition 3. (Behavior in Case of Trend Breaks)}

Let the data be generated by (1) with Assumptions 1 and 2 in place and let $\hat{\omega}_{m}^{2}$ denote again a consistent long-run variance estimator. Assume furthermore again that $g$ is continuous with $0<g(s)<\infty$.

(a) (Fixed Alternative) Let $\theta_{D}=\theta_{D, 1}$ for $t=1, \ldots,[r T]$ and $\theta_{D}=\theta_{D, 2}$, with $\theta_{D, 1} \neq$ $\theta_{D, 2}$, from $t=[r T]+1$ onwards, with

$$
\lim _{T \rightarrow \infty} \frac{1}{\sqrt{T}} \sum_{t=[r T]+1}^{T} D_{t}^{\prime}\left(\theta_{D, 1}-\theta_{D, 2}\right)= \pm \infty
$$

then it holds for any $c>0$ that

$$
\lim _{T \rightarrow \infty} \mathbb{P}\left(\tau_{m}\left(\widehat{H}^{m}, g, c\right)<\infty\right)=1
$$

(b) (Local Alternative) Let $\theta_{D}=\theta_{D, 1}$ for $t=1, \ldots,[r T]$ and $\theta_{D}=\theta_{D, 1}+G_{D}^{-1 \prime} \Delta_{\theta}$ from $t=[r T]+1$ onwards with

$$
\int_{r}^{1} D(z)^{\prime} d z \Delta_{\theta} \neq 0
$$

then the monitoring procedure has non-trivial local power. That means that for any $1-\alpha \geq \epsilon>0$ and the $0<c=c(\alpha, g)<\infty$ from Proposition 1 there exists $a$ $\Delta_{\theta}=\Delta_{\theta}(c, g)$ fulfilling (27) such that

$$
\lim _{T \rightarrow \infty} \mathbb{P}\left(\tau_{m}\left(\widehat{H}^{m}, g, c(\alpha, g)\right)<\infty\right) \geq 1-\epsilon
$$

The condition (25) is in case of polynomial trend functions fulfilled for all $\theta_{D, 1} \neq \theta_{D, 2}$, clearly for all $r<s \leq 1$ and not just for $s=1$ as used in (25). The condition (27) is not 
fulfilled only on an algebraic set. E.g. in case of $D_{t}=(1, t)^{\prime}$ it holds that $\int_{r}^{1} D(z)^{\prime} d z=$ $\left(1-r, \frac{1}{2}-\frac{1}{2} r^{2}\right)$ and thus the set of values for which $\Delta_{\theta} \in \mathbb{R}^{2}$ is orthogonal to this vector corresponds to a straight line in $\mathbb{R}^{2}$.

An analogous result as given above in (21) but now for the case of local trend breaks is, for the special case of $D_{t}=1$ and for $s>r \geq m$, given by:

$$
\begin{aligned}
\widehat{H}^{m}(s) \Rightarrow & \widehat{\mathcal{H}}^{m}(s)+2 \frac{\Delta_{\theta}}{\omega} \int_{r}^{s} \widehat{W}_{m}(z)(z-r) d z+ \\
& +\frac{\Delta_{\theta}^{2}}{\omega^{2}}\left(\frac{s^{3}}{3}-s^{2} r+s r^{2}-\frac{r^{3}}{3}\right) .
\end{aligned}
$$

Remark 4. In case that breaks occur in both the deterministic and stochastic component of $\left\{y_{t}\right\}_{t \in \mathbb{Z}}$, the behavior of our detector is a corresponding combination of the behavior discussed in Propositions 2 and 3. This implies that a rejection of the null hypothesis does not allow one to identify the source(s) of the break.

Remark 5. Our approach can also be employed for detecting bubbles. In the recent literature, a bubble is often characterized as a period where the behavior of a time series has switched from integrated to explosive behavior, compare Phillips et al. (2011). Thus, our procedure allows to detect (the beginning of) a bubble by considering the first difference of the series, since in case of no bubble the first differences are stationary, whereas in case of explosive behavior also the first differences exhibit explosive behavior.

Remark 6. In relation to the previous remark, with bubbles typically considered to be temporary rather than permanent phenomena, it has to be noted that our procedure will be consistent in detecting episodes of $\mathrm{I}(1)$ or explosive behavior, as long as these episodes have asymptotically positive length. E.g. in the case of only one period under the alternative it has to hold that this period occurs over a sub-sample of the form $\left[r_{1} T\right], \ldots,\left[r_{2} T\right]$ with $r_{1}<r_{2}$. It is immediate that consistency generalizes to multiple periods of this form. 
We close this section by looking at local asymptotic power (LAP) of the monitoring procedure. We consider LAP as discussed in Proposition 2(b) and against local-to-zero trend breaks as discussed in Proposition 3(b). The power curves are simulated similarly to the critical values. Discretized versions of the corresponding limiting distributions under the local alternatives are simulated. The limiting distribution for LAP against a unit root process is given in (21) and the limiting distribution in case of local trend breaks is given in (29). Again time series of length 1,000 are generated, with the number of replications given by 10,000. For each replication the errors $u_{t}$, and when considering Proposition 2(b) the $\xi_{t}$, are drawn as i.i.d. standard normal random variables independent of each other. The resulting values are then compared with the critical values and all test decisions are performed at the $5 \%$ level. All LAP curves are drawn for a grid of 21 equidistant values of $\delta$, in the interval $[0,100]$. Throughout we consider different combinations of the calibration period $m \in\{0.25,0.5,0.75\}$ and break point $r \geq m$ from the same set of values.

In Figure 1 we display local asymptotic power based on Proposition 2(b). The upper two plots consider the intercept only case and the lower two plots display the linear trend case. LAP is lower in the linear trend case compared to the intercept case. In each of the two sub-blocks of the figure the upper plot shows the effect of increasing $r$ relative to fixed $m=0.25$. As expected, LAP is decreasing with increasing $r$, since the period over which the integrated behavior can be detected is getting shorter and starts later. The lower two figures each display the effect of increasing the estimation sub-sample with increasing values of $m=r$. Here the effects are as expected for the intercept case, where LAP is decreasing with increasing values of $m=r$. The results are different in the linear trend case, where LAP is highest for $m=r=0.5$ and lowest for $m=r=0.25$.

In Figure 2 we display, based on the result in Proposition 3(b), LAP against breaks in the intercept (upper two plots) and breaks in the linear trend parameter (lower two plots). ${ }^{11}$ The structure of the plots is the same as in Figure 1 and also the findings are very similar.

\footnotetext{
${ }^{11}$ Note the different ranges of the horizontal axis for the two cases, with $\Delta_{\theta} \in[0,10]$ in case of an intercept break and $\Delta_{\theta} \in[0,100]$ in case of a break of the slope of the linear trend.
} 

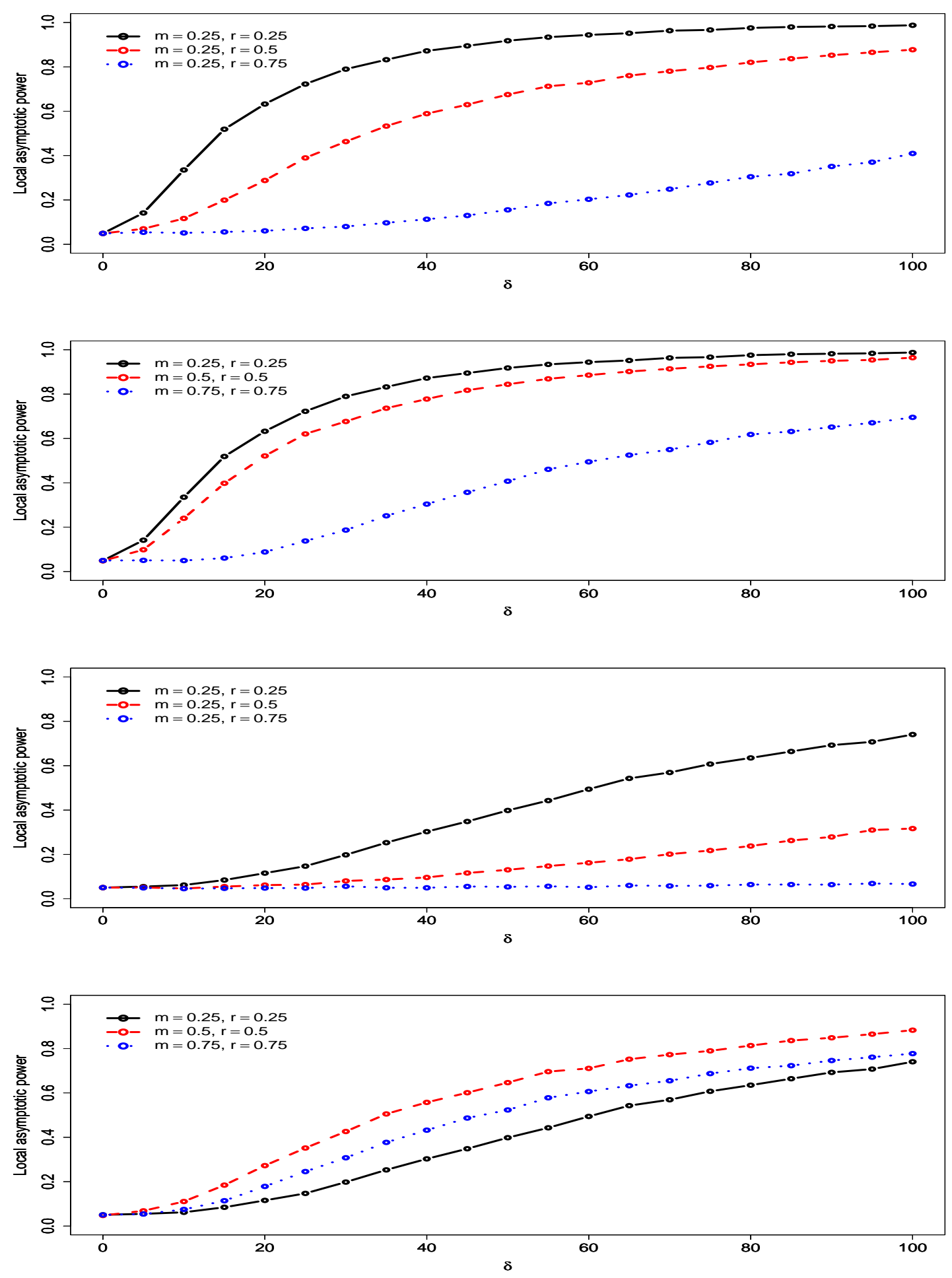

Figure 1: Local asymptotic power against I(1) alternatives. The upper two plots display the intercept case and the lower two plots the linear trend case. The plots show results for different combinations of $m$ and $r$. 
The only non-expected result again occurs in the lowest plot, where LAP for different values of $m=r$ in case of a break in the linear trend is displayed. In this case LAP is highest for $m=r=0.5$, whereas in the intercept only case LAP monotonously decreases in $m=r$.

\section{Monitoring Cointegration}

In this section we use the same ideas as discussed before to monitor cointegrating relationships using the following setup:

$$
\begin{aligned}
y_{t} & =D_{t}^{\prime} \theta_{D}+X_{t}^{\prime} \theta_{X}+u_{t} \\
X_{t} & =X_{t-1}+v_{t}
\end{aligned}
$$

with $y_{t}$ scalar, $D_{t} \in \mathbb{R}^{p}$ as before and $X_{t} k$-dimensional. The joint error vector process $\left\{\eta_{t}\right\}_{t \in \mathbb{Z}}$, with $\eta_{t}=\left(u_{t}, v_{t}^{\prime}\right)^{\prime}$, fulfills a similar assumption as $\left\{u_{t}\right\}_{t \in \mathbb{Z}}$ in the previous section:

Assumption 3. The stationary process $\left\{\eta_{t}\right\}_{t \in \mathbb{Z}}$ fulfills

$$
\frac{1}{\sqrt{T}} \sum_{t=1}^{[s T]} \eta_{t}=\frac{1}{\sqrt{T}} \sum_{t=1}^{[s T]}\left[\begin{array}{c}
u_{t} \\
v_{t}
\end{array}\right] \Rightarrow \Omega^{1 / 2} W(s)
$$

with $W(s)=\left[W_{u \cdot v}(s), W_{v}(s)^{\prime}\right]^{\prime} \in \mathbb{R}^{1+k}$ a vector of standard Brownian motions and $0<$ $\Omega<\infty$, with

$$
\Omega=\left[\begin{array}{ll}
\Omega_{u u} & \Omega_{u v} \\
\Omega_{v u} & \Omega_{v v}
\end{array}\right]:=\sum_{j=-\infty}^{\infty} \mathbb{E}\left(\eta_{t} \eta_{t-j}^{\prime}\right) .
$$

For our purposes it is convenient to use

$$
\Omega^{1 / 2}=\left[\begin{array}{cc}
\omega_{u \cdot v} & \lambda_{u v} \\
\mathbf{0} & \Omega_{v v}^{1 / 2}
\end{array}\right]
$$



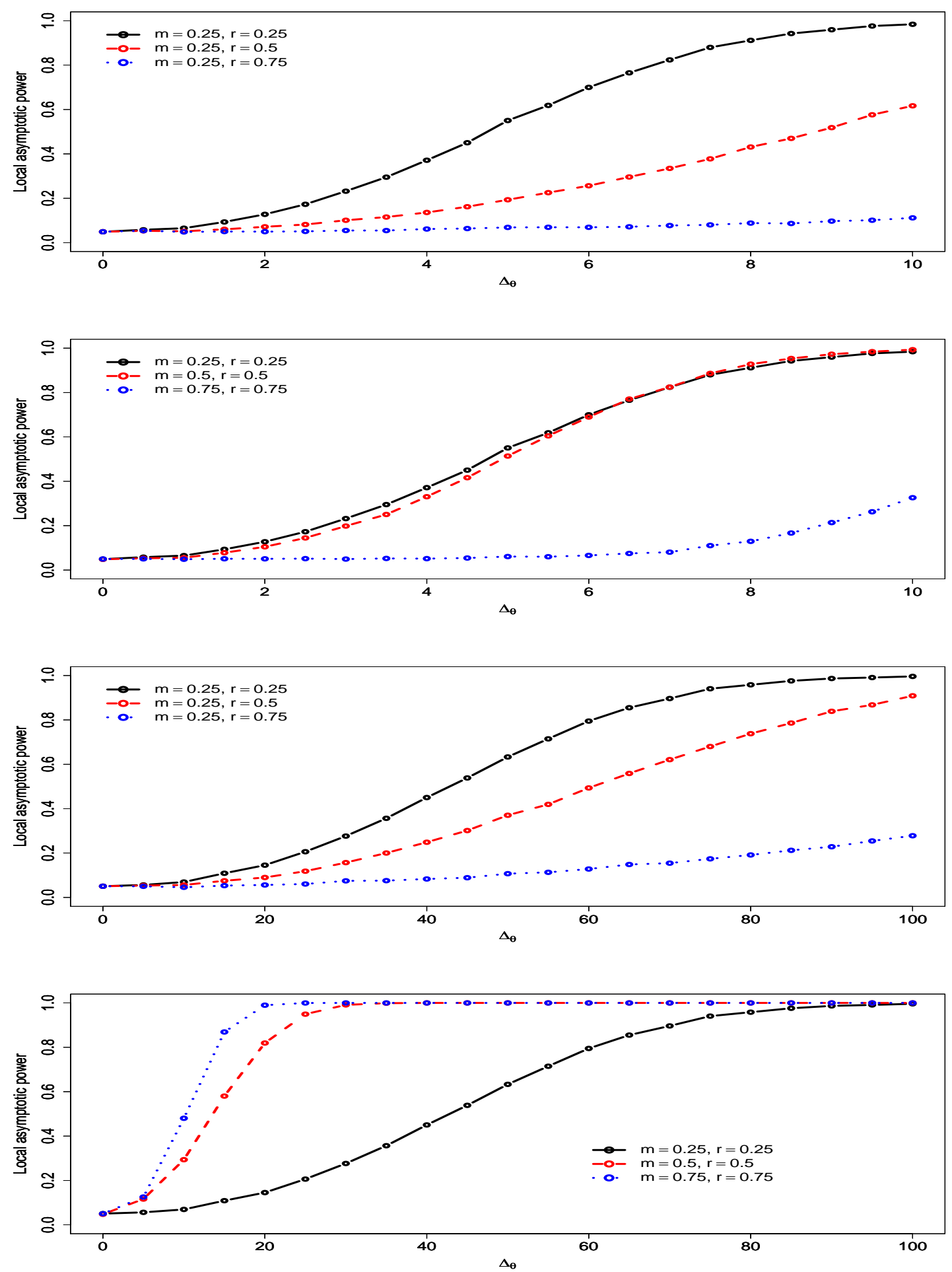

Figure 2: Local asymptotic power against breaks in deterministic components. The upper two plots display the case of a break in the intercept and the lower two plots display the case of a break in the linear trend. The plots show results for different combinations of $m$ and $r$. 
where $\omega_{u \cdot v}^{2}:=\Omega_{u u}-\Omega_{u v} \Omega_{v v}^{-1} \Omega_{v u}$ and $\lambda_{u v}:=\Omega_{u v}\left(\Omega_{v v}^{1 / 2}\right)^{-1}$.

The assumption $\Omega_{v v}>0$ excludes cointegration amongst the regressors and is typically required for the modified OLS estimation techniques available, including Fully Modified OLS (FM-OLS) of Phillips and Hansen (1990), Dynamic OLS (D-OLS) of Saikkonen (1991), and Integrated Modified OLS (IM-OLS) of Vogelsang and Wagner (2014). It is well-known that OLS estimation of $\theta=\left[\theta_{D}^{\prime}, \theta_{X}^{\prime}\right]^{\prime}$ in (30) is consistent, but that in general the limiting distribution of the OLS estimator depends on second order bias terms, which render asymptotic standard inference based on the OLS estimates infeasible. This problem occurs in particular when the regressors are not strictly exogenous, i.e. when the matrix $\Omega$ is not block-diagonal. ${ }^{12}$ The mentioned modified OLS estimators lead to limiting distributions of the parameters that are proportional to functionals of standard Brownian motions (which depend upon $D_{t}$ and the number of integrated regressors $k$ ) also in case of endogeneity. For brevity we abstain from explaining these well-known procedures here and just consider the residuals obtained from these estimation procedures as input in our monitoring procedure. ${ }^{13}$

We consider for illustration specifically the residuals of FM-OLS estimation, denoting the dependent variable used in FM-OLS by $y_{t}^{+}:=y_{t}-\Delta X_{t}^{\prime} \hat{\Omega}_{v v}^{-1} \hat{\Omega}_{v u}:{ }^{14}$

$$
\begin{aligned}
\hat{u}_{t, m}^{+} & :=y_{t}^{+}-D_{t}^{\prime} \hat{\theta}_{D, m}-X_{t}^{\prime} \hat{\theta}_{X, m} \\
& =y_{t}-\Delta X_{t}^{\prime} \hat{\Omega}_{v v}^{-1} \hat{\Omega}_{v u}-D_{t}^{\prime} \hat{\theta}_{D, m}-X_{t}^{\prime} \hat{\theta}_{X, m} \\
& =u_{t}-v_{t}^{\prime} \hat{\Omega}_{v v}^{-1} \hat{\Omega}_{v u}-D_{t}^{\prime}\left(\hat{\theta}_{D, m}-\theta_{D}\right)-X_{t}^{\prime}\left(\hat{\theta}_{X, m}-\theta_{X}\right),
\end{aligned}
$$

where $\hat{\theta}_{D, m}$ and $\hat{\theta}_{X, m}$ denote the FM-OLS coefficient estimates and $\hat{\Omega}\left(=\hat{\Omega}_{m}\right)$ denotes the long-run variance estimate, all computed from the pre-break sample $1, \ldots,[m T]{ }^{15}$

\footnotetext{
${ }^{12}$ In case of strict exogeneity, asymptotically valid inference can be based on the OLS estimates if serial correlation in $\left\{u_{t}\right\}_{t \in \mathbb{Z}}$ is handled appropriately using consistent long-run variance estimation.

${ }^{13}$ Only the less well-known IM-OLS estimator is briefly discussed below.

${ }^{14}$ Clearly, the construction of $y_{t}^{+}$using $\Delta X_{t}$ implies that the sample size available is $t=2, \ldots, T$. To simplify notational flow we nevertheless consider the sums below starting at $t=1$, by setting $\hat{u}_{1, m}^{+}=0$, with $\hat{u}_{t, m}^{+}$defined in the next equation. In the same way, D-OLS estimation leads to a reduced effective sample size due to the usage of leads and lags.

${ }^{15}$ In case the procedure is implemented using the D-OLS estimator, the residuals are defined
} 
Using consistent estimators of the long-run variances, ensured again by assuming to be in the framework covered by Jansson (2002), leads to a FCLT for the modified OLS residuals $\hat{u}_{t, m}^{+}$.

Lemma 2. Let the data be generated by (30) and (31) with Assumptions 1 and 3 in place and let $\hat{\Omega}$ be a consistent long-run variance estimator (required only for FM-OLS). Then it holds under the null hypothesis and for $m \leq s \leq 1$ for $T \rightarrow \infty$ for FM-OLS and D-OLS that

$$
\begin{aligned}
\frac{1}{\sqrt{T}} \sum_{t=1}^{[s T]} \hat{u}_{t, m}^{+} & \Rightarrow \omega_{u \cdot v}\left(W_{u \cdot v}(s)-\int_{0}^{s} J(z)^{\prime} d z\left(\int_{0}^{m} J(z) J(z)^{\prime} d z\right)^{-1} \int_{0}^{m} J(z) d W_{u \cdot v}(z)\right) \\
& =: \omega_{u \cdot v} \widehat{W}_{u \cdot v}(s)
\end{aligned}
$$

with $J(s):=\left[D(s)^{\prime}, W_{v}(s)^{\prime}\right]^{\prime}$.

Clearly, the process $\widehat{W}_{u \cdot v}(s)$ depends upon $D_{t}$, the number of integrated regressors $k$ and the pre-break fraction $m$, with these dependencies neglected for notational brevity henceforth.

Given the FCLT (36) for the partial sum process of the modified residuals, the detector for cointegration, using either the FM-OLS or the D-OLS estimator, is defined by

$$
\widehat{H}^{m,+}(s):=\frac{1}{\hat{\omega}_{u \cdot v}^{2}}\left(\frac{1}{T} \sum_{i=[m T]+1}^{[s T]}\left(\frac{1}{\sqrt{T}} \widehat{S}_{i}^{+}\right)^{2}-\frac{1}{T} \sum_{i=1}^{[m T]}\left(\frac{1}{\sqrt{T}} \widehat{S}_{i}^{+}\right)^{2}\right)
$$

where the scaling factor is now a consistent estimator $\hat{\omega}_{u \cdot v}^{2}=\hat{\Omega}_{u u}-\hat{\Omega}_{u v} \hat{\Omega}_{v v}^{-1} \hat{\Omega}_{v u}$ of the conditional long-run variance $\omega_{u \cdot v}^{2}$.

(using the same notation for the residuals and coefficient estimates) as $\hat{u}_{t, m}^{+}:=y_{t}-D_{t}^{\prime} \hat{\theta}_{D, m}-$ $X_{t}^{\prime} \hat{\theta}_{X, m}-\sum_{j=-k_{1}}^{k_{2}} \Delta X_{t-j}^{\prime} \hat{\Theta}_{j, m}$, or equivalently $\hat{u}_{t, m}^{+}=u_{t}-D_{t}^{\prime}\left(\hat{\theta}_{D, m}-\theta_{D}\right)-X_{t}^{\prime}\left(\hat{\theta}_{X, m}-\theta_{X}\right)-$ $\sum_{j=-k_{1}}^{k_{2}} \Delta X_{t-j}^{\prime} \hat{\Theta}_{j, m}$, with the matrices $\hat{\theta}_{D, m}, \hat{\theta}_{X, m}$ and $\hat{\Theta}_{j, m}$ being the OLS estimates from the regression $y_{t}=D_{t}^{\prime} \theta_{D}+X_{t}^{\prime} \theta_{X}+\sum_{j=-k_{1}}^{k_{2}} \Delta X_{t-j}^{\prime} \Theta_{j}+u_{t}$ estimated on the sample $1, \ldots,[m T]$. Whereas in FM-OLS estimation bandwidth and kernel have to be chosen, D-OLS estimation requires choosing the number of leads $k_{1}$ and lags $k_{2}$. Under appropriate assumptions concerning the asymptotic behavior of lag/lead choices the D-OLS residuals fulfill the same FCLT as the FM-OLS residuals. Asymptotically, therefore the usage of either estimator leads to the same monitoring procedure. 
All long-run variances and covariances required in the procedure (both for modified OLS parameter estimation as well as for scaling the detector) are based on the OLS residuals $\hat{u}_{t, m}$ stacked on top of the first differences of the regressors, i.e. upon $\hat{\eta}_{t}=\left[\hat{u}_{t, m}, v_{t}^{\prime}\right]^{\prime}$. Again the OLS estimation from which the parameter estimates and long-run variance estimates are computed uses observations $t=1, \ldots,[m T]$ only.

Given the definition of the detector for cointegration (37) the first result to be established is the asymptotic distribution of the detector under the null hypothesis.

Lemma 3. Let the data be generated by (30) and (31) with Assumptions 1 and 3 in place and let $\hat{\omega}_{u \cdot v}^{2}$ denote a consistent long-run variance estimator. Then it holds under the null hypothesis and for $m \leq s \leq 1$ for $T \rightarrow \infty$ for FM-OLS and D-OLS that

$$
\widehat{H}^{m,+}(s) \Rightarrow \int_{m}^{s} \widehat{W}_{u \cdot v}^{2}(z) d z-\int_{0}^{m} \widehat{W}_{u \cdot v}^{2}(z) d z=: \widehat{\mathcal{H}}^{m,+}(s)
$$

Alternatively, one can also base the cointegration monitoring procedure on the residuals of the recently proposed Integrated Modified OLS (IM-OLS) estimator of Vogelsang and Wagner (2014). A potential advantage of the IM-OLS estimator compared to FM-OLS and D-OLS is that for parameter estimation no kernel and bandwidth or lead and lag choices are required. The IM regression is given by

$$
S_{t}^{y}=S_{t}^{D^{\prime}} \theta_{D}+S_{t}^{X \prime} \theta_{X}+X_{t}^{\prime} \varphi+S_{t}^{u},
$$

with $S_{t}^{y}=\sum_{j=1}^{t} y_{j}$ denoting the partial sums, and similar definitions of $S_{t}^{D}$ and $S_{t}^{X}$.

We denote the corresponding OLS residuals, with estimation based upon the pre-break sample $1, \ldots,[m T]$ by (using the same notation for the coefficient estimates as before)

$$
\begin{aligned}
\hat{S}_{t, m}^{u} & :=S_{t}^{y}-S_{t}^{D^{\prime}} \hat{\theta}_{D, m}-S_{t}^{X \prime} \hat{\theta}_{X, m}-X_{t}^{\prime} \hat{\varphi}_{m} \\
& =S_{t}^{u}-X_{t}^{\prime} \hat{\varphi}_{m}-S_{t}^{D \prime}\left(\hat{\theta}_{D, m}-\theta_{D}\right)-S_{t}^{X \prime}\left(\hat{\theta}_{X, m}-\theta_{X}\right)
\end{aligned}
$$

Under the assumptions stated the following FCLT holds: 
Lemma 4. Let the data be generated by (30) and (31) with Assumptions 1 and 3 in place. Then it holds for $T \rightarrow \infty$ that

$$
\begin{aligned}
\frac{1}{\sqrt{T}} \sum_{t=2}^{[s T]} \Delta \hat{S}_{t, m}^{u} & \Rightarrow \omega_{u \cdot v}\left(W_{u \cdot v}(s)-f(s)^{\prime}\left(\int_{0}^{m} f(z) f(z)^{\prime} d z\right)^{-1} \int_{0}^{m}[F(m)-F(z)] d W_{u \cdot v}(z)\right) \\
& =: \omega_{u \cdot v} \tilde{P}_{m}(s),
\end{aligned}
$$

where $f(s):=\left[\int_{0}^{s} D(z)^{\prime} d z, \int_{0}^{s} W_{v}(z)^{\prime} d s, W_{v}(z)^{\prime}\right]^{\prime}$ and $F(s):=\int_{0}^{s} f(z) d z$.

Based upon the above result, the IM-OLS based detector is defined analogously as:

$$
\hat{I}^{m}(s):=\frac{1}{\hat{\omega}_{u \cdot v}^{2}}\left(\frac{1}{T} \sum_{i=[m T]+1}^{[s T]}\left(\frac{1}{\sqrt{T}} \hat{S}_{i, m}^{u}\right)^{2}-\frac{1}{T} \sum_{i=1}^{[m T]}\left(\frac{1}{\sqrt{T}} \hat{S}_{i, m}^{u}\right)^{2}\right)
$$

where the scaling is, as for the other detectors, based on a consistent estimator of $\omega_{u \cdot v}^{2}$. Note that the same estimator as for FM-OLS or D-OLS, i.e. the estimator based on the OLS residuals $\hat{u}_{t, m}$ stacked on top of the first differences of the regressors, is used. The asymptotic null behavior of the IM-OLS detector is given next.

Lemma 5. Let the data be generated by (30) and (31) with Assumptions 1 and 3 in place and let $\hat{\omega}_{u \cdot v}^{2}$ denote a consistent long-run variance estimator. Then it holds under the null hypothesis and for $m \leq s \leq 1$ for $T \rightarrow \infty$ for IM-OLS that

$$
\hat{I}^{m}(s) \Rightarrow \int_{m}^{s} \tilde{P}_{m}(z)^{2} d z-\int_{0}^{m} \tilde{P}_{m}(z)^{2} d z=: \mathcal{I}^{m}(s)
$$

As for the stationarity monitoring procedure, it can be shown that under the null hypothesis for given weighting function $g(s)$, there exist critical values $c=c(\alpha, g(s))$, such that the detection time is finite with probability equal to the pre-specified level $\alpha$.

Proposition 4. Let the data be generated by (30) and (31) with Assumptions 1 and 3 in place, let long-run variance estimation be carried out consistently and assume again that $g$ is continuous with $0<g(s)<\infty$. Then, under the null hypothesis there exists for any 
given $0<\alpha<1$ critical values $c=c(\alpha, g)$, depending upon estimation method, such that

$$
\lim _{T \rightarrow \infty} \mathbb{P}\left(\tau_{m}\left(\widehat{H}^{m,+}, g, c(\alpha, g)\right)<\infty\right)=\alpha
$$

in case that FM-OLS or D-OLS is used, respectively

$$
\lim _{T \rightarrow \infty} \mathbb{P}\left(\tau_{m}\left(\widehat{I}^{m}, g, c(\alpha, g)\right)<\infty\right)=\alpha
$$

in case IM-OLS is used.

Remark 7. Note that for given weighting function $g$ the critical values are identical for the FM-OLS and D-OLS based detectors, but are different for the IM-OLS based detector.

The critical values depend again also on the weighting function $g(s)$, which we again choose as $g(s)=s^{3}$ in the intercept case $D_{t}=1$ and $g(s)=s^{5}$ in the linear trend case $D_{t}=(1, t)^{\prime}$. Critical values for these two cases are provided for FM-OLS \& D-OLS and for IM-OLS for one and two regressors in Appendix B in Tables 6 to $13 .{ }^{16}$ The simulations are performed analogously to the ones described above in Section 2 for the critical values for the stationarity monitoring procedure.

It remains to establish the behavior of the monitoring procedure under alternatives. In fact there are now three dimensions of structural change against which the procedures are shown to have power. First, changes in the behavior of $\left\{u_{t}\right\}_{t \in \mathbb{Z}}$, where we consider exactly the same alternatives as above in Proposition 2. Second, again similar to before, we consider the behavior against breaks in the parameters corresponding to the deterministic component. Third, we now additionally consider the behavior against breaks in the slope coefficients corresponding to the integrated regressors. For all three cases we consider fixed and local alternatives.

\footnotetext{
${ }^{16}$ The supplementary material contains critical values for three and four regressors.
} 


\section{Proposition 5. (Consistency and Local Asymptotic Power)}

Let the data be generated by (30) and (31) with Assumption 1 in place and $\left\{\eta_{t}\right\}_{t \in \mathbb{Z}}$ fulfilling Assumption 3 until $[r T]$, with $m \leq r<1$. Furthermore, assume that long-run variance estimation is performed consistently using observations $1, \ldots,[m T]$ and assume again that $g$ is continuous with $0<g(s)<\infty$. Denote with $\widehat{F}^{m}(s)$ either $\widehat{H}^{m,+}(s)$ or $\widehat{I}^{m}(s)$.

(a) Let $\left\{u_{t}\right\}_{t \in \mathbb{Z}}$ be an I(1) process (as specified in $H_{1}$ ) from $[r T]+1$ onwards. Then the monitoring procedures are consistent, i.e. for any $c>0$ it holds that

$$
\lim _{T \rightarrow \infty} \mathbb{P}\left(\tau_{m}\left(\widehat{F}^{m}, g, c(\alpha, g)\right)<\infty\right)=1
$$

(b) Let $\left\{u_{t}\right\}_{t \in \mathbb{Z}}$ be as specified in (17) from $[r T]+1$ onwards. Then the monitoring procedures have non-trivial local power. That means, for any $1-\alpha \geq \epsilon>0$ and the $0<c=c(\alpha, g)<\infty$ from Proposition 4 there exists a $0<\delta=\delta(c, g)<\infty$ such that

$$
\lim _{T \rightarrow \infty} \mathbb{P}\left(\tau_{m}\left(\widehat{F}^{m}, g, c(\alpha, g)\right)<\infty\right) \geq 1-\epsilon
$$

\section{Proposition 6. (Behavior in Case of Trend Breaks)}

Let the data be generated by (30) and (31) with Assumptions 1 and 3 in place. Furthermore, assume that long-run variance estimation is performed consistently using observations $1, \ldots,[m T]$ and and assume again that $g$ is continuous with $0<g(s)<\infty$. Denote with $\widehat{F}^{m}(s)$ either $\widehat{H}^{m,+}(s)$ or $\widehat{I}^{m}(s)$.

(a) (Fixed Alternative) Let $\theta_{D}=\theta_{D, 1}$ for $t=1, \ldots,[r T]$ and $\theta_{D}=\theta_{D, 2}$, with $\theta_{D, 1} \neq$ $\theta_{D, 2}$, from $t=[r T]+1$ onwards, with

$$
\lim _{T \rightarrow \infty} \frac{1}{\sqrt{T}} \sum_{t=[r T]+1}^{T} D_{t}^{\prime}\left(\theta_{D, 1}-\theta_{D, 2}\right)= \pm \infty
$$


then it holds for any $c>0$ that

$$
\lim _{T \rightarrow \infty} \mathbb{P}\left(\tau_{m}\left(\widehat{F}^{m}, g, c\right)<\infty\right)=1
$$

(b) (Local Alternative) Let $\theta_{D}=\theta_{D, 1}$ for $t=1, \ldots,[r T]$ and $\theta_{D}=\theta_{D, 1}+G_{D}^{-1 \prime} \Delta_{\theta}$ from $t=[r T]+1$ onwards with

$$
\int_{r}^{1} D(z)^{\prime} d z \Delta_{\theta} \neq 0
$$

then the monitoring procedure has non-trivial local power. That means, for any $1-\alpha \geq \epsilon>0$ and the $0<c=c(\alpha, g)<\infty$ from Proposition 4 there exists $a$ $\Delta_{\theta}=\Delta_{\theta}(c, g)$ fulfilling (50) such that

$$
\lim _{T \rightarrow \infty} \mathbb{P}\left(\tau_{m}\left(\widehat{F}^{m}, g, c(\alpha, g)\right)<\infty\right) \geq 1-\epsilon
$$

Compared to the discussion in the previous section, there is now the additional possibility of breaks in the slope coefficients $\theta_{X}$, which are in a sense equivalent to changes in the behavior of the $\left\{u_{t}\right\}_{t \in \mathbb{Z}}$. Consider for simplicity the case $\theta_{X}=\theta_{X, 1}$ for $t=1, \ldots,[r T]$ and $\theta_{X}=\theta_{X, 2}$, with $\theta_{X, 1} \neq \theta_{X, 2}$, for $t=[r T]+1, \ldots, T$. In this case we can write for $t>[r T]$

$$
\begin{aligned}
y_{t} & =D_{t}^{\prime} \theta_{D}+X_{t}^{\prime} \theta_{X, 2}+u_{t} \\
& =D_{t}^{\prime} \theta_{D}+X_{t}^{\prime} \theta_{X, 1}+X_{t}^{\prime}\left(\theta_{X, 2}-\theta_{X, 1}\right)+u_{t} .
\end{aligned}
$$

Clearly, this implies that in the residual process starting from $[r T]$ onwards an integrated process given by $X_{t}^{\prime}\left(\theta_{X, 2}-\hat{\theta}_{X, 1, m}\right)$ is present. This component remains present as an $\mathrm{I}(1)$ process also in the limit due to consistency of $\hat{\theta}_{X, 1, m} \rightarrow \theta_{X, 1} \neq \theta_{X, 2}$. Consequently, in case of a break in the slope parameters, the residual process is an I(1) process. Therefore, the asymptotic behavior in case of slope breaks is similar to the case discussed in Proposition 5. We therefore have a very similar result, where local alternatives are now 
of the form $\theta_{X, 2}=\theta_{X, 1}+\frac{1}{T} \Delta_{\theta}$.

\section{Proposition 7. (Behavior in Case of Slope Breaks)}

Let the data be generated by (30) and (31) with Assumptions 1 and 3 in place. Furthermore, assume that long-run variance estimation is performed consistently using observations $1, \ldots,[m T]$ and and assume again that $g$ is continuous with $0<g(s)<\infty$. Denote with $\widehat{F}^{m}(s)$ either $\widehat{H}^{m,+}(s)$ or $\widehat{I}^{m}(s)$.

(a) Considering fixed alternatives of the form $\theta_{X}=\theta_{X, 1}$ for $t=1, \ldots,[r T]$ and $\theta_{X}=$ $\theta_{X, 2}$ for $t=[r T]+1, \ldots, T$, with $\theta_{X, 1} \neq \theta_{X, 2}$ leads to a similar result as in part (a) of Proposition 5.

(b) Considering local alternatives of the form $\theta_{X}=\theta_{X, 1}$ for $t=1, \ldots,[r T]$ and $\theta_{X}=$ $\theta_{X, 1}+\frac{1}{T} \Delta_{\theta}$, with $\Delta_{\theta} \neq 0$, for $t=[r T]+1, \ldots, T$ leads to a similar result as in part (b) of Proposition 5.

Remark 8. Remarks 3 to 6 apply analogously to the cointegration monitoring procedures as well.

We again close this section by considering local asymptotic power which is simulated analogously to the LAP simulations in the previous section, i.e. the number of replications is 10,000 and the time series considered are of length 1,000. All random variables are i.i.d. standard normal. The limiting distribution for LAP as discussed in Proposition 5(b) is based on the FCLTs under local alternatives given in (60) for FM-OLS and D-OLS and (61) for IM-OLS in Appendix A. These are the input to obtain a limiting distribution similar to the limiting distribution given in (21) used above to study LAP when monitoring stationarity. Also local asymptotic power against trend breaks (Proposition 6) is simulated in the same way with the corresponding FCLTs given in (64) and (65) in Appendix A that can be used to obtain limiting distributions similar to (29). Finally, local asymptotic power against slope breaks (Proposition 7) is based on the FCLTs given in (68) and (69) in Appendix A. Also for this case the FCLTs allow to establish the limiting distributions of the detectors under the local alternative. Considering these results 
in detail leads to exactly the same observations as in the previous section with respect to the dependence of LAP on the signal-to-noise ratio. As an extension of this fact it turns out that LAP against slope breaks increases with the variance of the regressors. Similarly to the simulations in the previous section we set all signal-to-noise ratios equal to one. It is also clear that in addition to the dependence upon the deterministic component, LAP now also depends upon the number of integrated regressors, as illustrated in Figure 3. As expected, LAP decreases with an increasing number of regressors. Consequently, all other results displayed are for the case of only one integrated regressor.

In Figure 4 we display LAP against local I(1) alternatives in case of intercept and linear trends included in the model, where we consider again the same combinations of $m$ and $r$ as in the previous section. The upper two plots correspond to FM-OLS and D-OLS and the lower two plots correspond to IM-OLS. The results show that LAP is lower for IM-OLS, which is as expected given the results of Vogelsang and Wagner (2014) concerning the relative conditional efficiency of FM-OLS over IM-OLS. ${ }^{17}$ The practical usefulness of IM-OLS stems from the lower finite sample size distortions that it implies compared to FM-OLS, as illustrated in the following section where we consider finite sample simulations. With respect to changing values of $m$ and $r$ all methods have similar LAP rankings, which coincide with the rankings found in the previous section.

In Figure 5 we display local asymptotic power results against breaks in the intercept, with the same structure of the figure as in Figure 4. The ordering of LAP as a function of $m=r$ (in the second and fourth plot) differs between FM-OLS/D-OLS and IM-OLS. For IM-OLS LAP increases with increasing $m=r$, whereas for the other two methods LAP is, as in the previous section, highest for $m=r=0.5$.

\footnotetext{
${ }^{17}$ The results are similar, with the differences smaller, in case of the model with intercept only. Additional results, including also results for breaks in the slope parameter, are available in supplementary material. The findings are, as expected, very similar to the ones for local I(1) alternatives.
} 

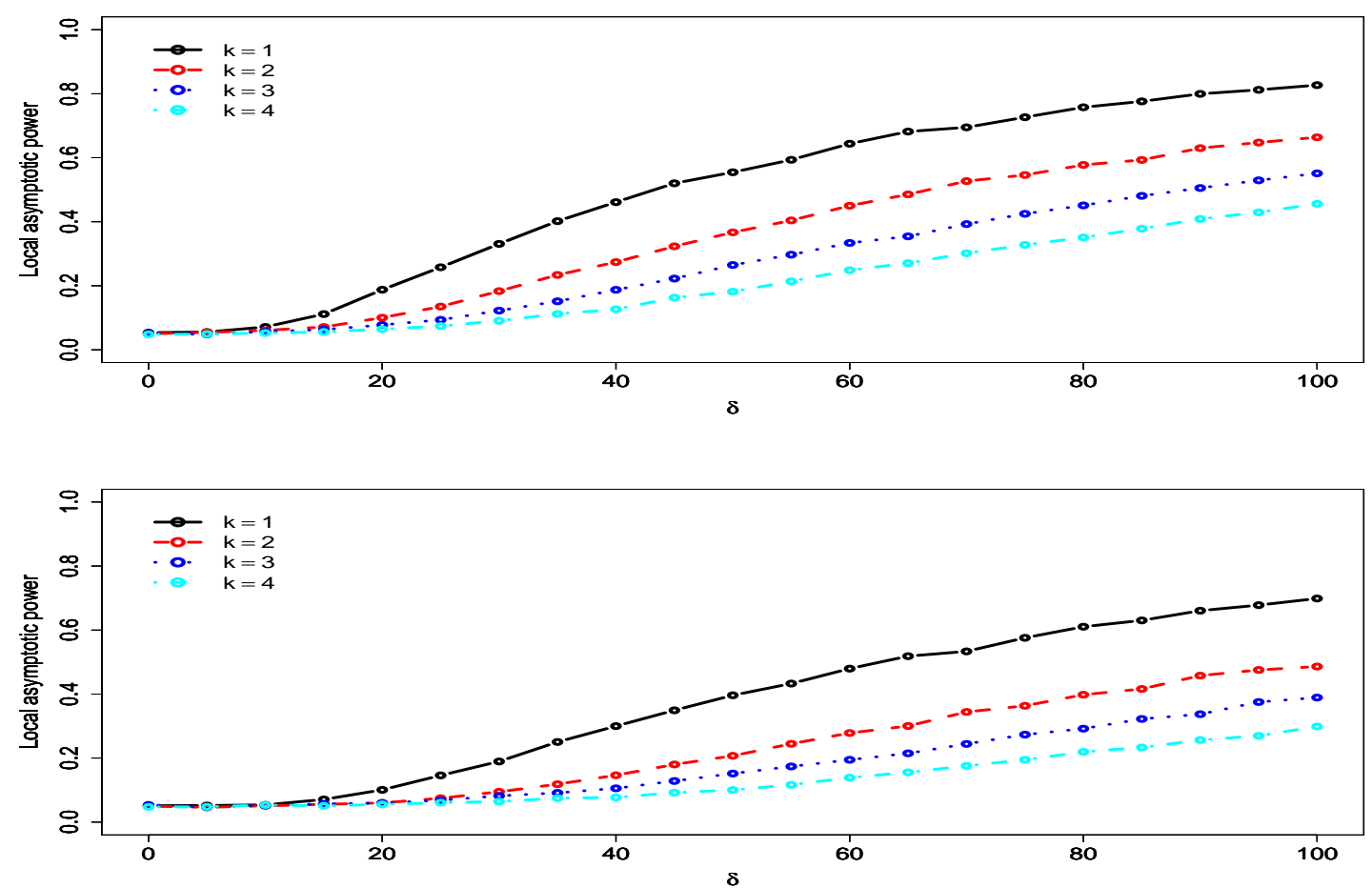

Figure 3: Local asymptotic power for $k=1, \ldots, 4$ regressors for monitoring cointegration for the case with intercept. The upper plot corresponds to FM-OLS \& D-OLS and the lower plot to IM-OLS. The plots show results for $m=r=0.25$. 

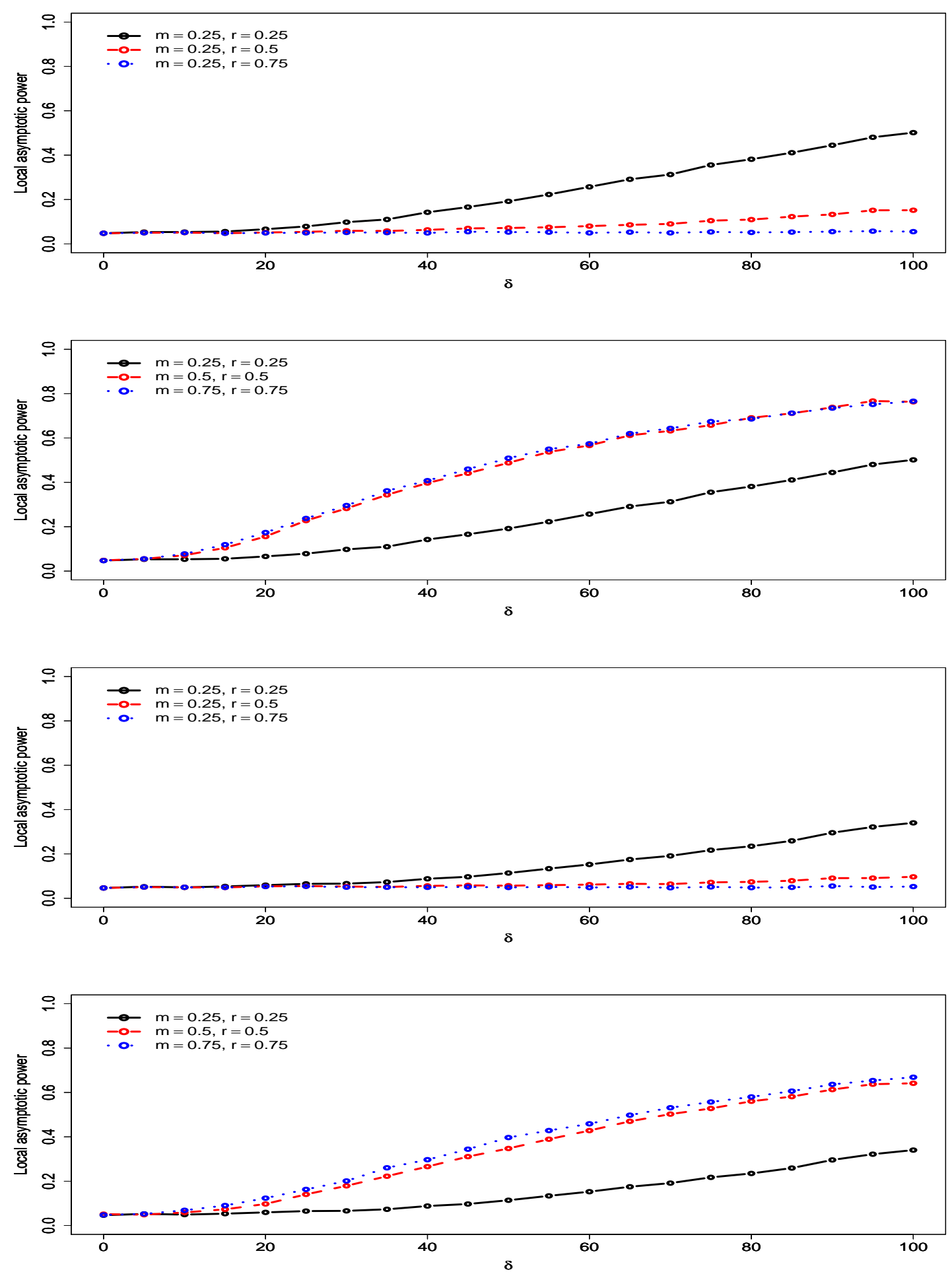

Figure 4: Local asymptotic power for monitoring cointegration for the case with intercept and linear trend. The upper two plots correspond to FM-OLS \& D-OLS and the lower two plots to IM-OLS. The plots show results for different combinations of $m$ and $r$. 

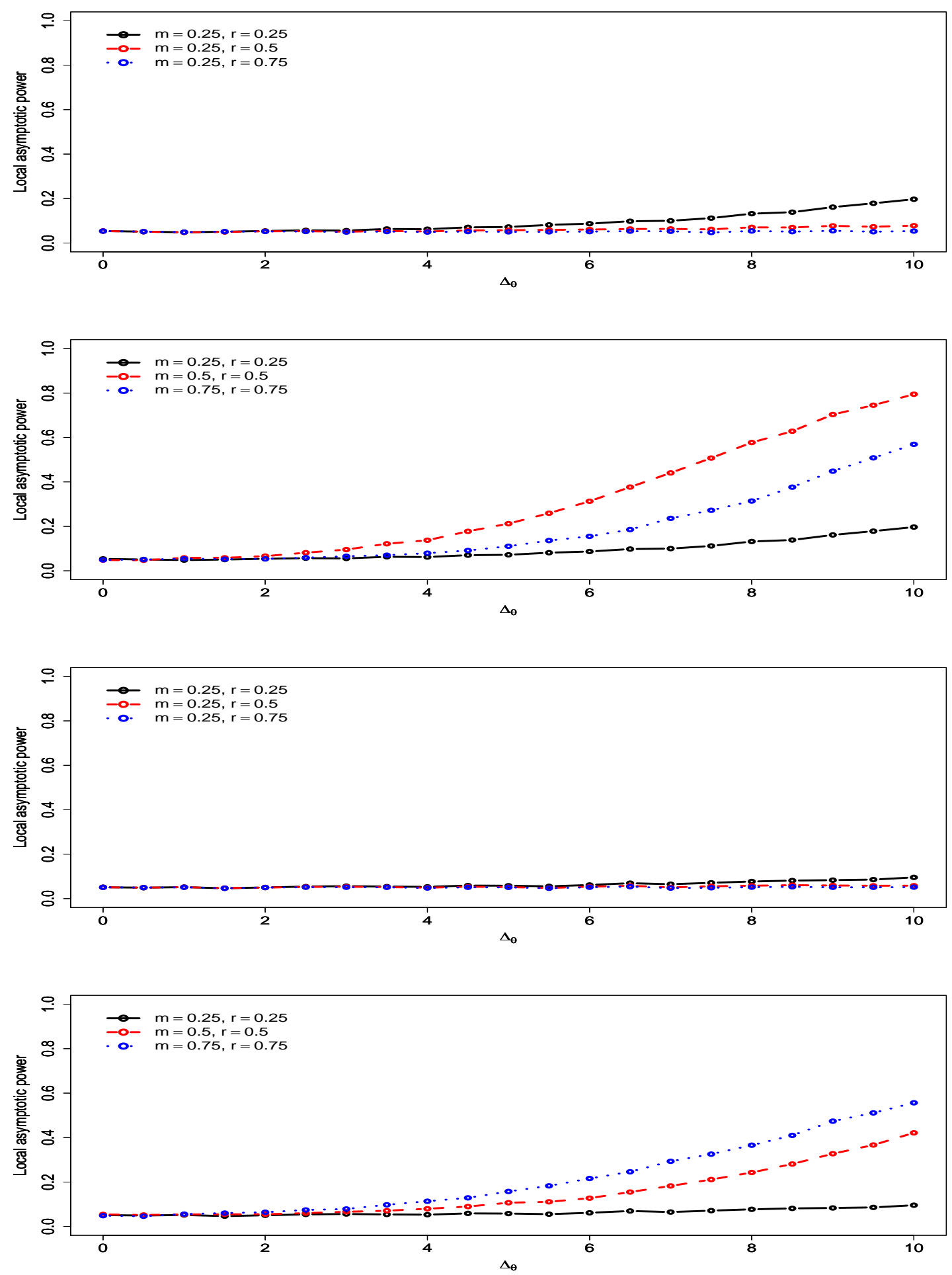

Figure 5: Local asymptotic power against break in intercept for monitoring cointegration for the case with intercept. The upper two plots correspond to FM-OLS \& D-OLS and the lower two plots to IM-OLS. The plots show results for different combinations of $m$ and $r$. 


\section{Finite Sample Performance}

In this section we investigate the performance of the monitoring procedure by means of a small simulation study. For the sake of brevity we only consider cointegration monitoring. We consider a data generating process similar to Vogelsang and Wagner (2014), i.e. we consider (under the null hypothesis):

$$
\begin{aligned}
y_{t} & =\mu+\gamma t+x_{1 t} \beta_{1}+x_{2 t} \beta_{2}+u_{t}, \\
x_{i t} & =x_{i, t-1}+v_{i t}, \quad x_{i 0}=0, \quad i=1,2
\end{aligned}
$$

where

$$
\begin{aligned}
& u_{t}=\rho_{1} u_{t-1}+\varepsilon_{t}+\rho_{2}\left(e_{1 t}+e_{2 t}\right), \quad u_{0}=0, \\
& v_{i t}=e_{i t}+0.5 e_{i, t-1}, \quad i=1,2,
\end{aligned}
$$

where $\varepsilon_{t}, e_{1 t}$ and $e_{2 t}$ are i.i.d. standard normal random variables independent of each other. The parameter values chosen are $\mu=3, \beta_{1}, \beta_{2}, \gamma=1$. Due to space limitations we will only briefly comment on the corresponding results for the intercept only case $(\gamma=0)$ but abstain from showing the analogous graphs and tables (which are available as supplementary material). The values for $\rho_{1}$ and $\rho_{2}$ are chosen from the set $\{0.0,0.3,0.6,0.9\}$. The parameter $\rho_{1}$ controls serial correlation in the regression error and is set to $\rho_{1}=1$ under the alternative of $\mathrm{I}(1)$ errors, whereas the parameter $\rho_{2}$ controls whether the regressors are endogenous $\left(\rho_{2} \neq 0\right)$ or not $\left(\rho_{2}=0\right)$.

Both, parameter estimation as well as the computation of the detector require the choice of kernel and bandwidth for long-run variance estimation. We use the data dependent bandwidth chosen according to Andrews (1991) and the Bartlett kernel. The D-OLS estimator is implemented using the information criterion based lead and lag length choice developed in Kejriwal and Perron (2008), where we use the more flexible version discussed in Choi and Kurozumi (2012) in which the numbers of leads and lags included are not 
$\rho_{1}=\rho_{2}=0$

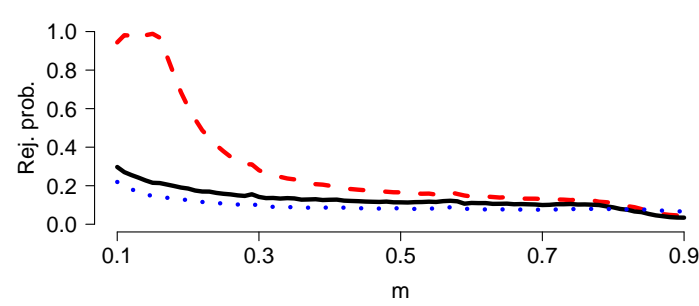

$\rho_{1}=\rho_{2}=0.6$

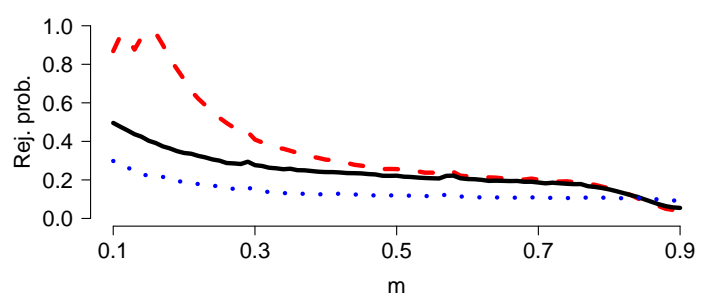

$\rho_{1}=\rho_{2}=0.3$

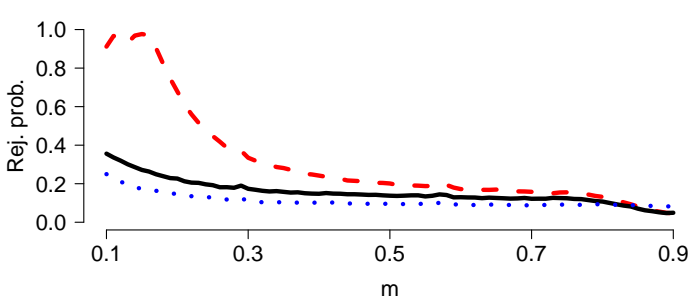

$\rho_{1}=\rho_{2}=0.9$

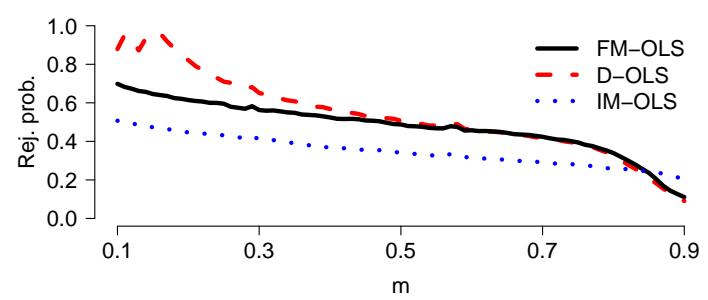

Figure 6: Empirical null rejection probabilities for monitoring cointegration for the case with intercept and linear trend for a grid of values of $m$ and $T=200$.

restricted to be equal. The considered sample sizes are $T=200,500$ and the number of replications is 10,000. All tests are performed at the $5 \%$ nominal level.

We start by considering the empirical null rejection probabilities for a grid of 81 values given by $m=0.1,0.11, \ldots, 0.9$ for $\rho_{1}, \rho_{2}=0,0.3,0.6,0.9$ and $T=200$ in Figure 6 and $T=500$ in Figure 7. Several main patterns in line with expectations emerge: First, the size distortions decrease with increasing $m$ and increasing sample size $T$. The exception to this general pattern is D-OLS and $T=200$ where size distortion are very high and partly increasing for small values of $m$ before they start decreasing. Second, larger values of $\rho_{1}, \rho_{2}$ lead to increasing size distortions and the larger $\rho_{1}, \rho_{2}$ are, the more beneficial is a larger value of $m$ to mitigate the size distortions. Third, the largest size distortions occur for D-OLS and typically the smallest ones for IM-OLS, with the largest differences for small values of $m$. The larger $\rho_{1}, \rho_{2}$ the bigger is the performance advantage of IM-OLS over the two other methods. Grosso modo the observed size distortions are comparable to the size distortions observed for parameter tests in Vogelsang and Wagner (2014). Let us note for completeness that size distortions are smaller when considering the intercept only case. 
$\rho_{1}=\rho_{2}=0$

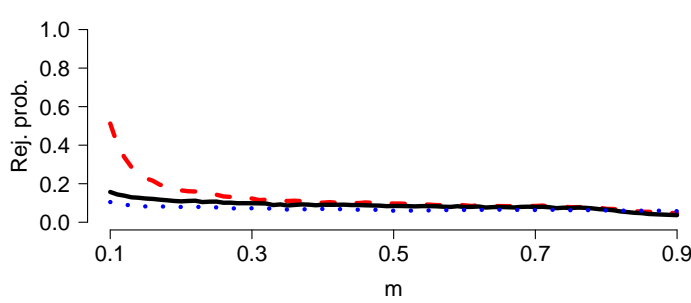

$\rho_{1}=\rho_{2}=0.6$

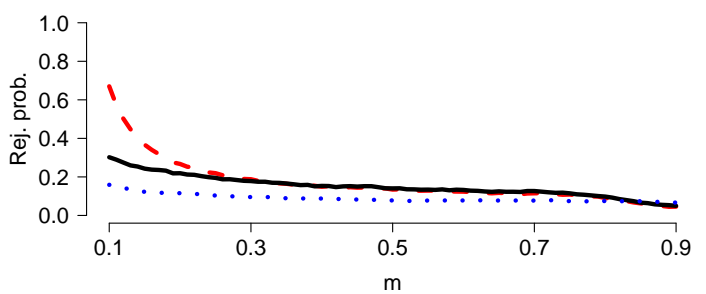

$\rho_{1}=\rho_{2}=0.3$

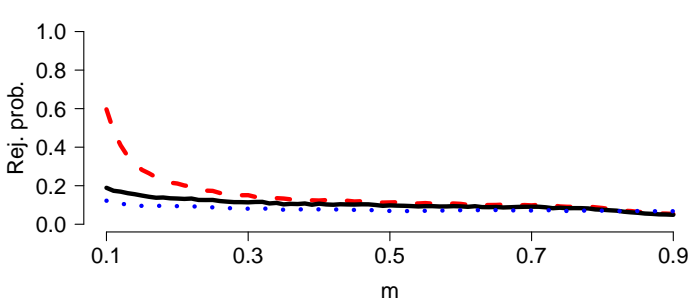

$\rho_{1}=\rho_{2}=0.9$

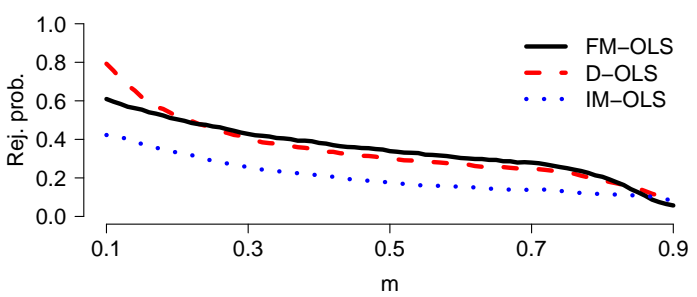

Figure 7: Empirical null rejection probabilities for monitoring cointegration for the case with intercept and linear trend for a grid of values of $m$ and $T=500$.

Let us next consider power against $\mathrm{I}(1)$ errors from sample fraction $[r T]$ for calibration fraction $[m T]$. We consider the values $0.25,0.5,0.75$ for both $m$ and $r$. Thus, we also include cases where $m>r$, i.e. where the break occurs in the calibration period. It can be shown, most easily for stationarity monitoring, that in case $m>r$, the order of the detector is given by $O_{p}\left(\frac{T}{b_{T}}\right)$, with $b_{T}$ denoting the bandwidth chosen. Given that in case of I(1) errors, i.e. under the alternative, typically large bandwidths $b_{T}$ are chosen, one can expect a low divergence rate of the detector in the $m>r$ case and thus low power in finite samples. This is exactly what happens. The results are shown for $T=200$ in Table 1 and for $T=500$ in Table 2 .

In addition to the above observation, two more (to be expected) findings are that power increases with the sample size $T$ and decreases with $\rho_{1}, \rho_{2}$. For any given constellation of $T$ and $\rho_{1}, \rho_{2}$ power is largest for $m=r$, compared to situations where $m \neq r$. For $m=r$, power is the larger the larger $m$ and $r$ are. In case that $m>r$, i.e. when the break occurs in the calibration period, power is also increasing with the sample size. In this case, however, power is decreasing in the difference between $m$ and $r$. This finding reflects the fact that asymptotically the term that is subtracted in the numerator of the 


\begin{tabular}{|c|c|c|c|c|c|c|c|c|c|c|}
\hline & & \multicolumn{3}{|c|}{$m=0.25$} & \multicolumn{3}{|c|}{$m=0.5$} & \multicolumn{3}{|c|}{$m=0.75$} \\
\hline & $r$ & 0.25 & 0.5 & 0.75 & 0.25 & 0.5 & 0.75 & 0.25 & 0.5 & 0.75 \\
\hline \multirow{3}{*}{0} & FM & 0.60 & 0.52 & 0.43 & 0.21 & 0.87 & 0.56 & 0.06 & 0.34 & 0.89 \\
\hline & $\mathrm{D}$ & 0.20 & 0.49 & 0.44 & 0.07 & 0.82 & 0.57 & 0.05 & 0.27 & 0.87 \\
\hline & IM & 0.51 & 0.47 & 0.43 & 0.17 & 0.80 & 0.54 & 0.06 & 0.25 & 0.82 \\
\hline \multirow{3}{*}{0.3} & FM & 0.45 & 0.47 & 0.39 & 0.14 & 0.79 & 0.51 & 0.05 & 0.23 & 0.83 \\
\hline & $\mathrm{D}$ & 0.13 & 0.42 & 0.39 & 0.06 & 0.71 & 0.51 & 0.05 & 0.16 & 0.80 \\
\hline & IM & 0.39 & 0.43 & 0.40 & 0.12 & 0.71 & 0.50 & 0.05 & 0.17 & 0.75 \\
\hline \multirow{3}{*}{0.6} & FM & 0.21 & 0.31 & 0.29 & 0.07 & 0.56 & 0.38 & 0.05 & 0.10 & 0.65 \\
\hline & $\mathrm{D}$ & 0.08 & 0.34 & 0.33 & 0.05 & 0.53 & 0.42 & 0.05 & 0.09 & 0.65 \\
\hline & IM & 0.23 & 0.35 & 0.37 & 0.08 & 0.53 & 0.43 & 0.05 & 0.09 & 0.60 \\
\hline \multirow{3}{*}{0.9} & $\overline{F M}$ & 0.06 & 0.07 & 0.09 & 0.05 & 0.09 & 0.10 & 0.05 & 0.05 & 0.15 \\
\hline & $\mathrm{D}$ & 0.05 & 0.08 & 0.12 & 0.05 & 0.09 & 0.13 & 0.05 & 0.05 & 0.15 \\
\hline & IM & 0.06 & 0.09 & 0.14 & 0.05 & 0.09 & 0.14 & 0.05 & 0.05 & 0.13 \\
\hline
\end{tabular}

Table 1: Empirical rejection probabilities for monitoring cointegration for the case with intercept and linear trend for $T=200$ : Size corrected power against I(1) breaks.

detector is under the alternative a function of the difference $m-r$, rather than of $m$ as under the null. In case that $m \leq r$ power is decreasing with increasing $r$, which merely reflects the fact that a smaller sub-sample is available under the alternative for detecting the structural change from I(0) to I(1) behavior.

The differences in power between FM-OLS and IM-OLS are minor, whereas power of D-OLS is markedly smaller in case $r=0.25$. Clearly, this latter finding cannot be predicted by LAP, which is by construction identical between FM-OLS and D-OLS. This finding reflects the well-known fact that D-OLS leads to coefficient estimators with larger variances in small samples. Comparing FM-OLS and IM-OLS shows a slight tendency that power for IM-OLS is marginally lower than for FM-OLS, and in case that $r$ is large marginally lower than for D-OLS, which is in line with the LAP analysis. In case of large values of $\rho_{1}, \rho_{2}$, however, size corrected power is sometimes higher for IMOLS. These findings concerning power, however, have to be seen in conjunction with the partly substantially lower size distortions when using IM-OLS compared to FM-OLS or in particular D-OLS. All findings analogously hold for the intercept only case, with power being typically higher in the intercept only case compared to the intercept and linear trend case. 


\begin{tabular}{|c|c|c|c|c|c|c|c|c|c|c|}
\hline & & \multicolumn{3}{|c|}{$m=0.25$} & \multicolumn{3}{|c|}{$m=0.5$} & \multicolumn{3}{|c|}{$m=0.75$} \\
\hline & 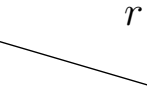 & 0.25 & 0.5 & 0.75 & 0.25 & 0.5 & 0.75 & 0.25 & 0.5 & 0.75 \\
\hline \multirow{3}{*}{0} & $\mathrm{FM}$ & 0.94 & 0.57 & 0.47 & 0.59 & 0.99 & 0.61 & 0.11 & 0.72 & 0.99 \\
\hline & $\mathrm{D}$ & 0.91 & 0.56 & 0.48 & 0.54 & 0.98 & 0.63 & 0.09 & 0.70 & 0.99 \\
\hline & IM & 0.89 & 0.52 & 0.48 & 0.49 & 0.97 & 0.60 & 0.08 & 0.63 & 0.98 \\
\hline \multirow{3}{*}{0.3} & FM & 0.86 & 0.53 & 0.45 & 0.47 & 0.97 & 0.58 & 0.08 & 0.61 & 0.98 \\
\hline & $\mathrm{D}$ & 0.82 & 0.52 & 0.45 & 0.40 & 0.96 & 0.58 & 0.07 & 0.59 & 0.98 \\
\hline & IM & 0.80 & 0.49 & 0.45 & 0.38 & 0.95 & 0.56 & 0.07 & 0.51 & 0.95 \\
\hline \multirow{3}{*}{0.6} & FM & 0.64 & 0.44 & 0.38 & 0.24 & 0.90 & 0.50 & 0.06 & 0.39 & 0.93 \\
\hline & $\mathrm{D}$ & 0.62 & 0.48 & 0.41 & 0.22 & 0.91 & 0.52 & 0.06 & 0.41 & 0.93 \\
\hline & IM & 0.63 & 0.45 & 0.43 & 0.23 & 0.87 & 0.53 & 0.06 & 0.34 & 0.89 \\
\hline \multirow{3}{*}{0.9} & FM & 0.09 & 0.17 & 0.19 & 0.05 & 0.27 & 0.24 & 0.05 & 0.06 & 0.41 \\
\hline & $\mathrm{D}$ & 0.09 & 0.21 & 0.24 & 0.06 & 0.32 & 0.30 & 0.05 & 0.06 & 0.46 \\
\hline & IM & 0.10 & 0.25 & 0.32 & 0.06 & 0.32 & 0.37 & 0.05 & 0.06 & 0.43 \\
\hline
\end{tabular}

Table 2: Empirical rejection probabilities for monitoring cointegration for the case with intercept and linear trend for $T=500$ : Size corrected power against $\mathrm{I}(1)$ breaks.

We next consider power against breaks in the trend or slope parameters. For brevity we only display results for the case $\rho_{1}, \rho_{2}=0.9$, i.e. the worst results from our set of values for $\rho_{1}, \rho_{2}$. All figures have the same structure and display in the left column the graphs for $m=0.25$ and $r \in\{0.25,0.5,0.75\}$ and in the right column the graphs for $m=r \in\{0.25,0.5,0.75\}$.

For trend breaks we consider a grid with 21 points and mesh 0.1 for the trend slope $\gamma \in[1,3]$ (including the null) displayed on the horizontal axis in Figure 8 for $T=200$ and in Figure 9 for $T=500$. Three main observations emerge: First, power is decreasing with increasing $r$ for given $m$ (left column). Second, power is increasing with increasing $m=r$ (right column). Third, power is very similar for FM-OLS and IM-OLS and is in some cases lower for D-OLS. The differences across estimation methods get smaller for increasing sample size.

For slope breaks we consider the case that $\beta_{1}, \beta_{2}$ both change their value from 1 to some value larger than 1, where we consider the same grid of 21 points as for trend breaks above, i.e. $\beta_{i} \in[1,3]$ (including the null). The results for $T=200$ are displayed in Figure 10 and for $T=500$ in Figure 11. The results are qualitatively very similar to the findings in case of a trend break. Note for completeness that power against slope 

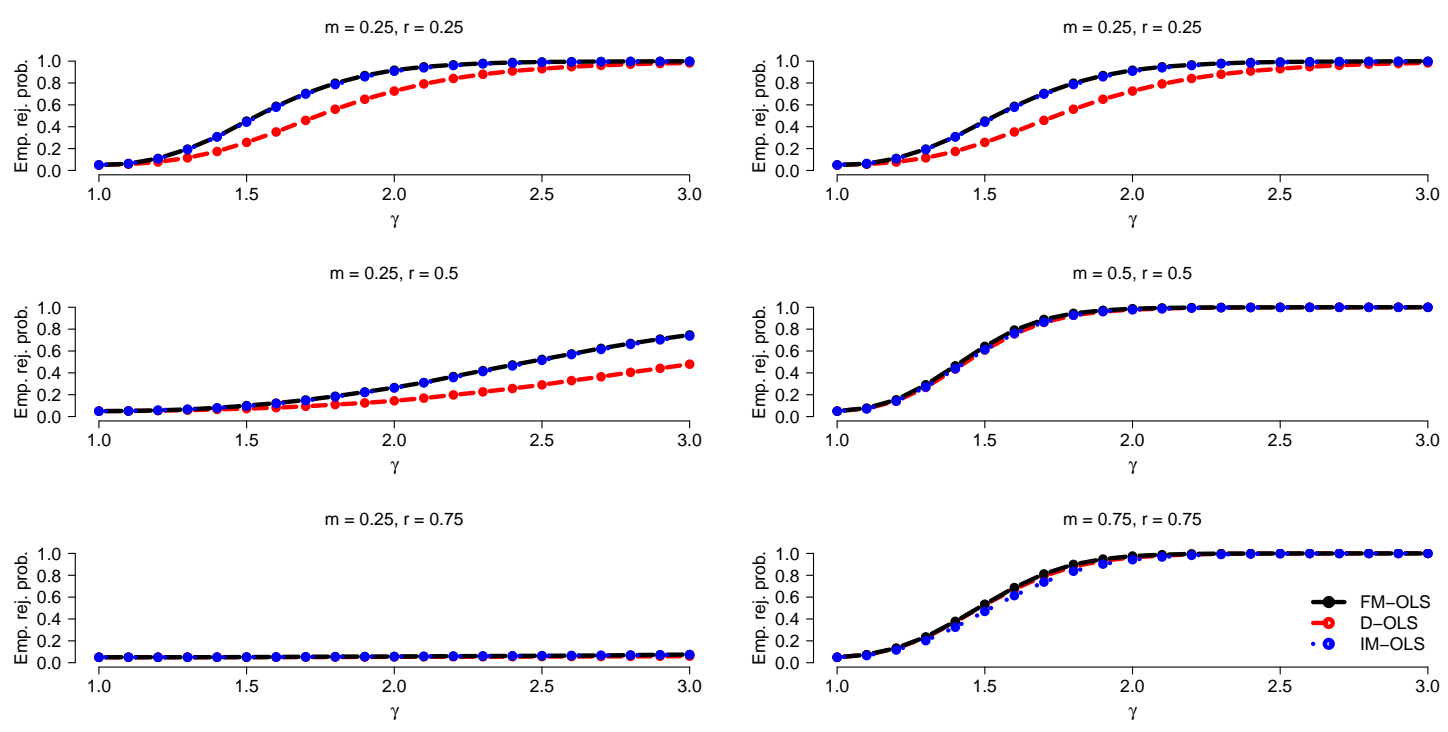

Figure 8: Empirical rejection probabilities for monitoring cointegration for the case with intercept and linear trend for $T=200, \rho_{1}=\rho_{2}=0.9$ : Size corrected power against trend breaks.
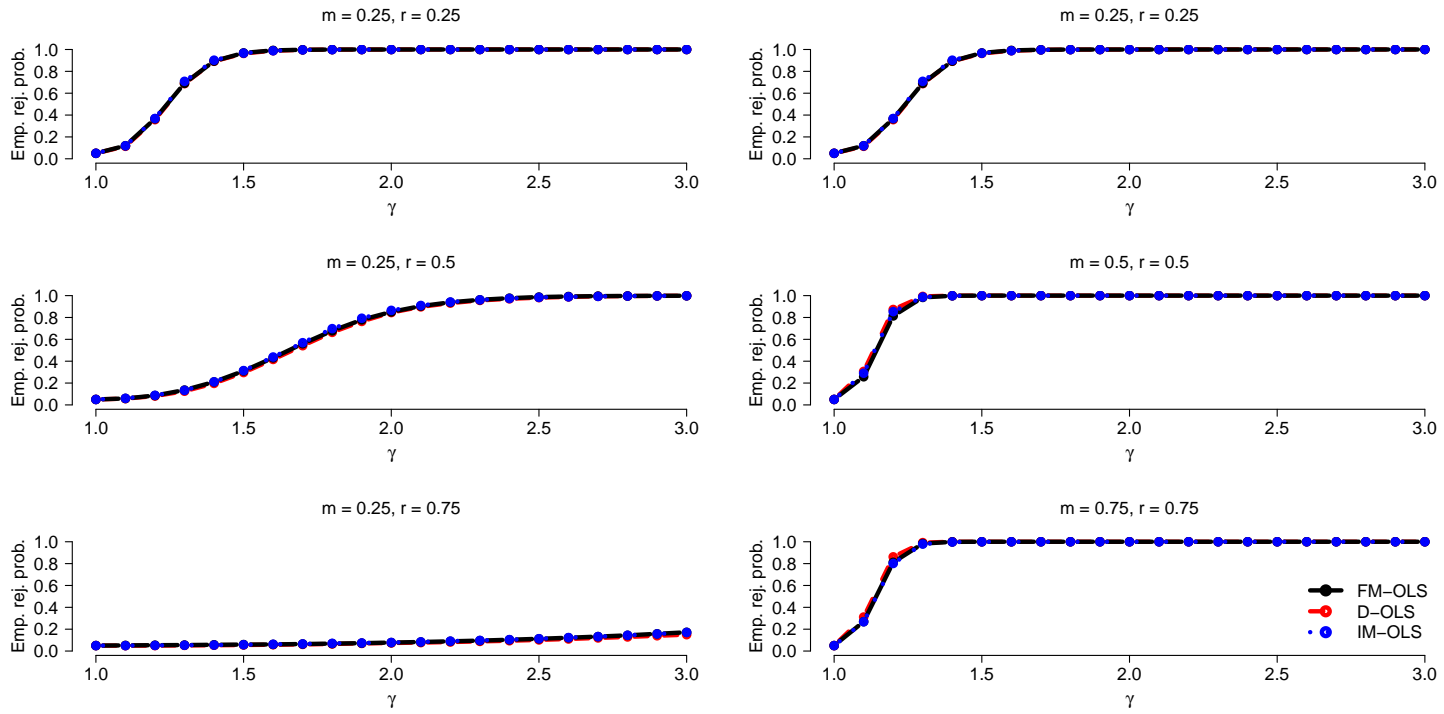

Figure 9: Empirical rejection probabilities for monitoring cointegration for the case with intercept and linear trend for $T=500, \rho_{1}=\rho_{2}=0.9$ : Size corrected power against trend breaks. 

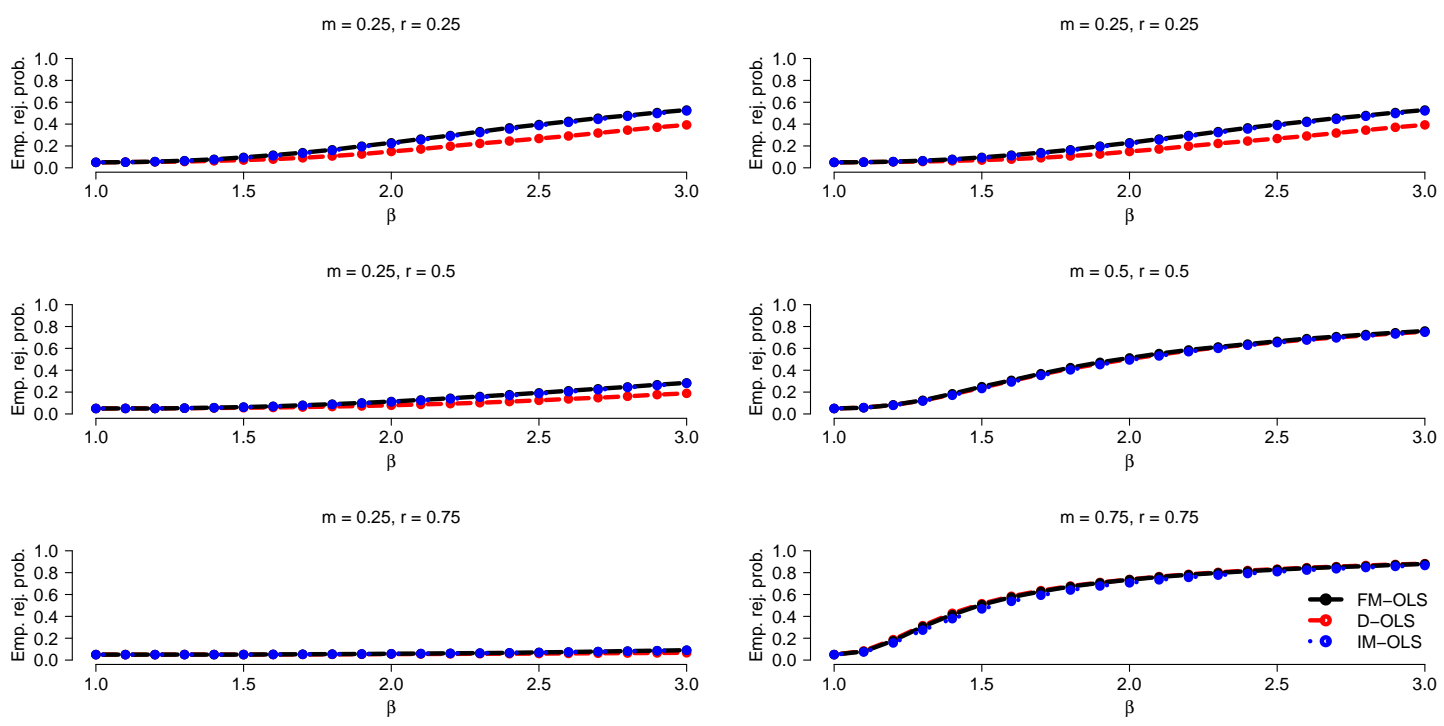

Figure 10: Empirical rejection probabilities for monitoring cointegration for the case with intercept and linear trend for $T=200, \rho_{1}=\rho_{2}=0.9$ : Size corrected power against slope breaks.

breaks (of similar magnitude) is slightly higher in the intercept only case compared to the intercept and linear trend case.

Note that the similarities and differences between LAP and finite sample simulations discussed above, when considering power against I(1) errors exist as well for the case of trend and slope breaks.

Finally we investigate the estimated detection times against I(1) breaks. These are shown in Figures $12\left(\rho_{1}, \rho_{2}=0.9\right)$ and $13\left(\rho_{1}, \rho_{2}=0\right)$ for $T=200$ and in Figures $14\left(\rho_{1}, \rho_{2}=0\right)$ and $15\left(\rho_{1}, \rho_{2}=0.9\right)$ for $T=500$. We display the detection times in the form of BoxWhiskers plots. The numbers below the abbreviated names of the methods indicate the null rejection probabilities given in Tables 1 and 2. Thus, the different Box-Whiskers plots are based on different numbers of replications because of a different number of rejections across different methods, sample sizes and $\rho$-parameters.

The structure of the six graphs within one figure corresponds to the structure of the power figures with respect to the combinations of $m$ and $r$ displayed. By construction, detection occurs on average with delay. An increasing sample size leads to a - ceteris paribus - more concentrated distribution of the estimated detection times (based on a 

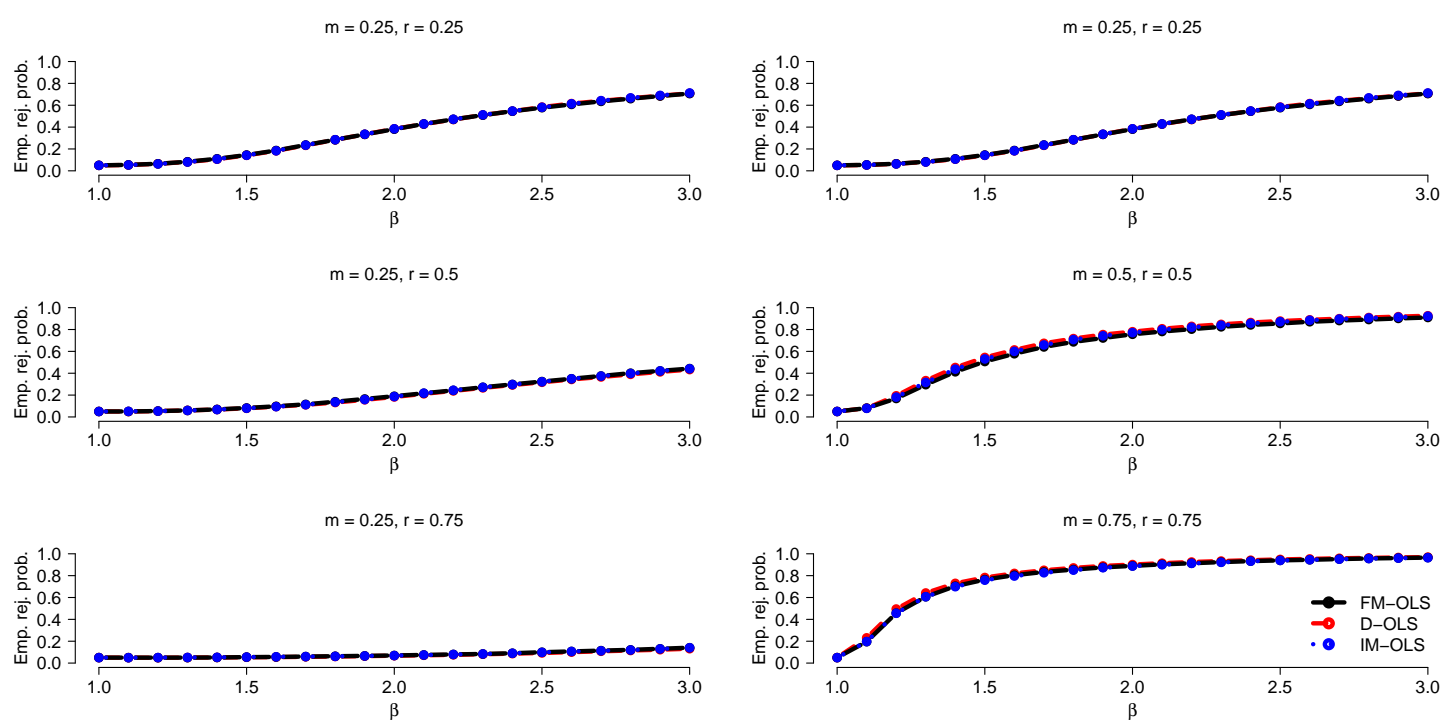

Figure 11: Empirical rejection probabilities for monitoring cointegration for the case with intercept and linear trend for $T=500, \rho_{1}=\rho_{2}=0.9$ : Size corrected power against slope breaks.

larger number of observations), but does not necessarily lead to smaller average delays. As expected, increasing endogeneity and error serial correlation lead to increasing detection times, i.e. bigger delays. For fixed $m=0.25$ increasing values of $r$ lead to decreasing delays. For $m=0.25$ and $r=0.75$ detection often occurs already prior to the structural change. Increasing values of $m=r$ also lead to smaller delays of detecting the structural change.

When comparing the three methods, there is no clear ranking with respect to delay. FMOLS and IM-OLS lead to very similar detection time distributions, whereas the D-OLS detection times look rather different from the other two. These differences are more marked in cases where the rejection probabilities (i.e. powers) of D-OLS are sizeably smaller than for the other two methods. The detection delays are partly substantial, in particular for $\rho_{1}, \rho_{2}=0.9$. Obtaining a better understanding of the impact of the weighting function on the expected delays is consequently a topic of future research, notwithstanding the complications outlined in Section 2. 

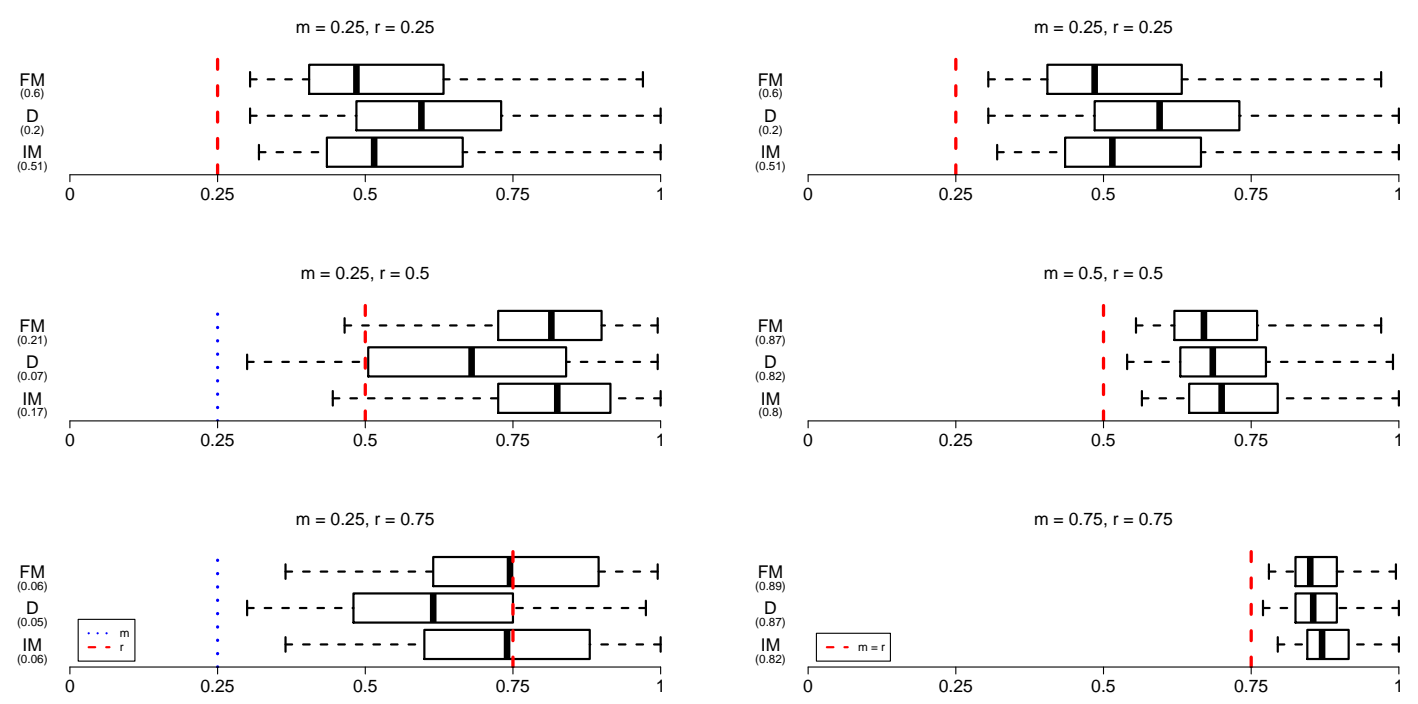

Figure 12: Detection times when monitoring cointegration for the case with intercept and linear trend for $T=200, \rho_{1}=\rho_{2}=0$ : Detection times for I(1) breaks.
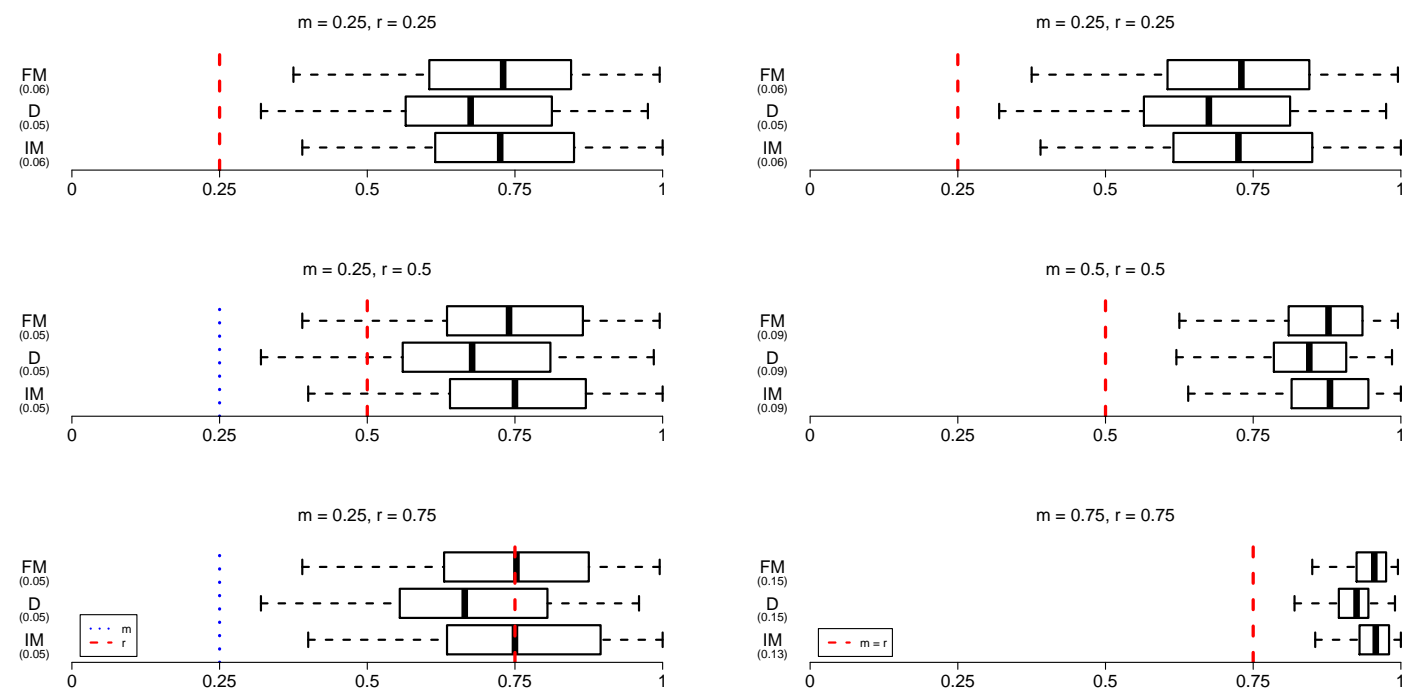

Figure 13: Detection times when monitoring cointegration for the case with intercept and linear trend for $T=200, \rho_{1}=\rho_{2}=0.9$ : Detection times for I(1) breaks. 

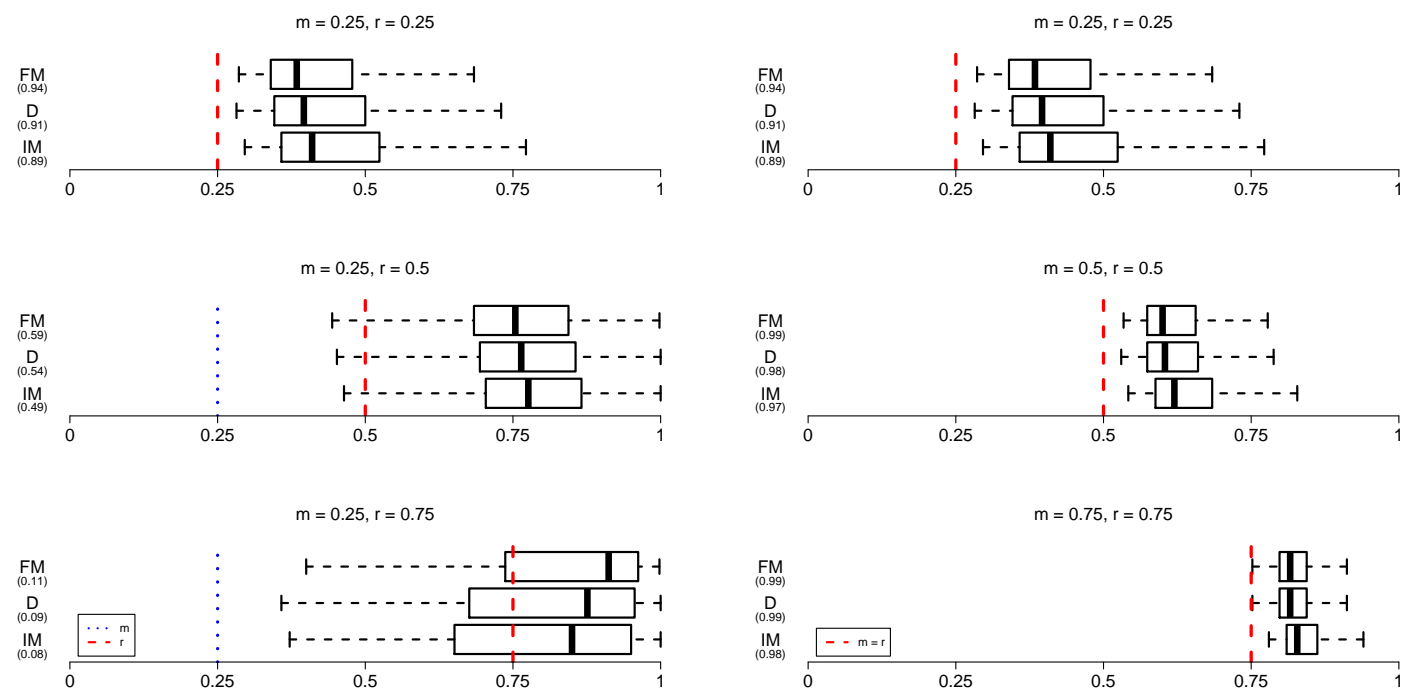

Figure 14: Detection times when monitoring cointegration for the case with intercept and linear trend for $T=500, \rho_{1}=\rho_{2}=0$ : Detection times for I(1) breaks.
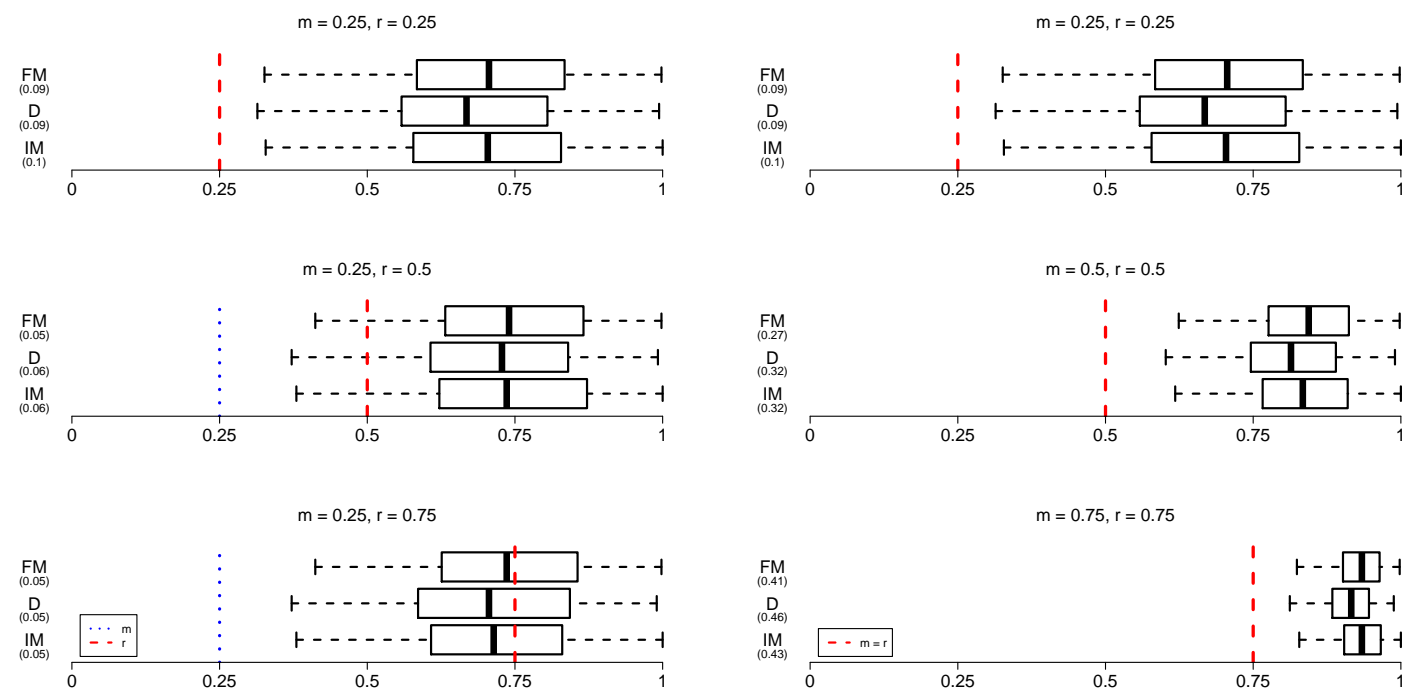

Figure 15: Detection times when monitoring cointegration for the case with intercept and linear trend for $T=500, \rho_{1}=\rho_{2}=0.9$ : Detection times for I(1) breaks. 


\section{EMpirical Illustration}

We apply the monitoring procedure to investigate daily CDS spreads data for Austria, Cyprus, France and Germany for maturities one, three, five, seven and ten years, see Figure 16. Stationary or nonstationary behavior of CDS spreads during financial crises has been investigated e.g. by Dieckmann and Plank (2011) who find mixed evidence in this respect. The mixed evidence may not least be driven by changing stationarity behavior over time such that an application of our monitoring procedure appears potentially useful. The time span chosen ranges from April 3, 2009 to August 1, 2012, giving a total of $T=869$ observations. We try to exclude the immediate consequences of the Lehman brothers bankruptcy on September 15, 2008 and therefore take as starting date the day after a G20 summit held in London on April 1-2, 2009 that is considered to have had stabilizing influences on the financial markets. Our sample period ranges until August 1, 2012, which is the date when Cyprus was downgraded from investment grade BB+ to BB by Standard \& Poor's. The calibration period consists of the first $10 \%$ of the observations, i.e. ranges from April 3, 2009 to July 31, 2009 and is indicated by the vertical lines in Figure 16. ${ }^{18}$

Graphical inspection of the data already allows for a few important observations. First, within each country the series corresponding to the different maturities move together quite closely, displaying something like a "term structure" of CDS spreads. In this respect it is interesting to note that for Cyprus the term structure is inverse after the two big jumps occurring on July 27 and August 26, 2011. ${ }^{19}$ After these jumps the level of the series is much larger for Cyprus than for the other three countries that also experience upward jumps around the same time. This, of course, illustrates the well-known fact that there are not only within-country dependencies across different maturities, but that there is also a large amount of cross-country co-movements of CDS spreads series, compare also

\footnotetext{
${ }^{18}$ The results are very robust and do not change at all when considering $m=0.2$ instead. Note that for values of $m$ larger than 0.2 the KPSS stationarity test leads to rejection of the null of stationarity on the calibration sample for all countries and maturities at the $1 \%$ level. From this perspective therefore, the choice of the calibration period appears to be in line with data properties.

${ }^{19}$ On July 27, 2011 Moody's downgraded Cyprus to Baa1 after an explosion at a marine base.
} 

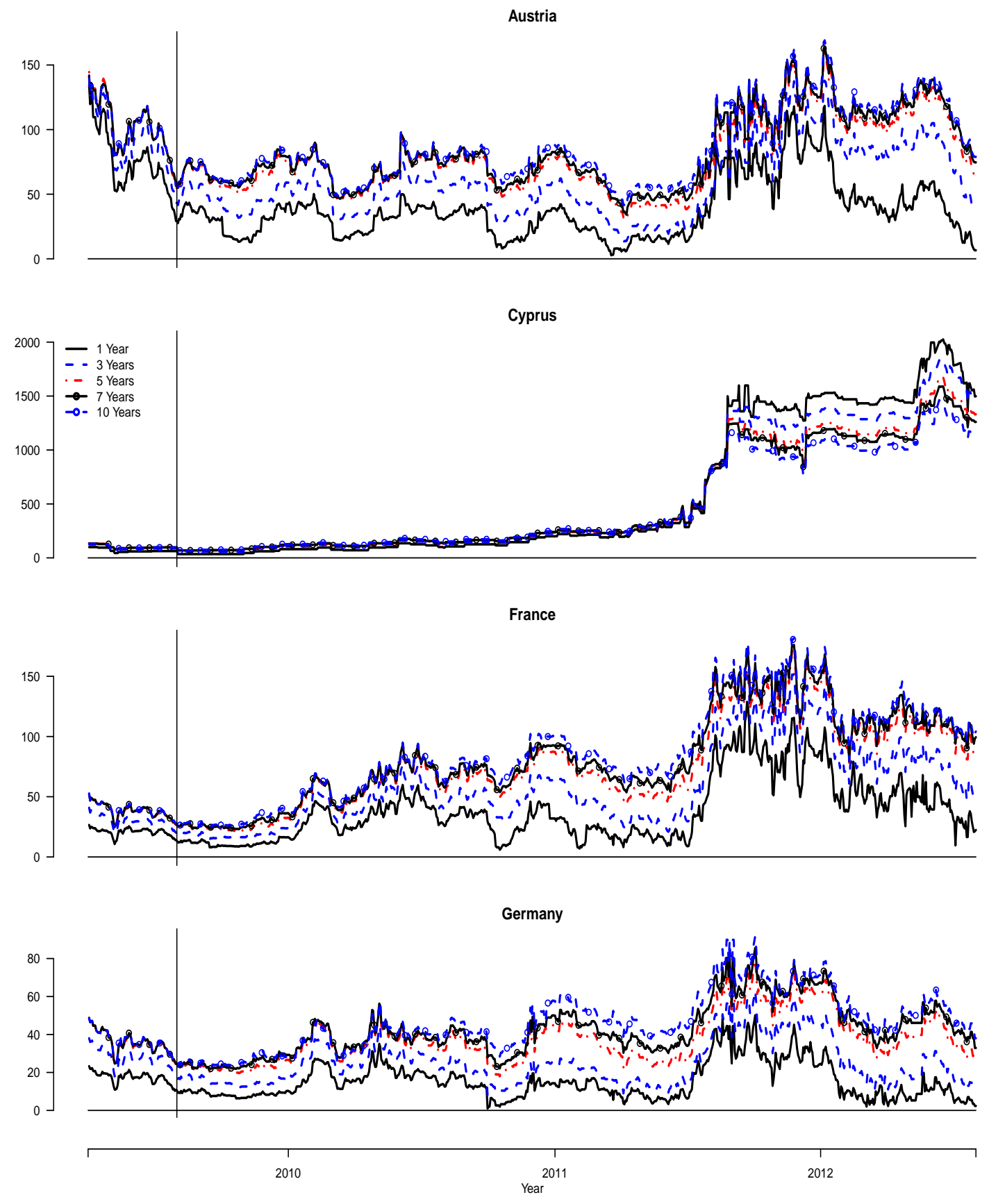

Figure 16: Daily CDS spreads series. 
Dieckmann and Plank (2011). ${ }^{20}$

The test results are displayed in Table 3 and indicate a rejection of the null hypothesis of stationarity at the $5 \%$ significance level for all series considered. By construction, the estimated break dates are later for lower values of $\alpha$. The break dates (at the $5 \%$ significance level) are between April 27, 2010 (France, ten years) and February 1, 2011 (Germany, one year). All these dates precede the discussed major level shifts in the CDS series that occurred in summer 2011. This is remarkable, given the delays observed in the finite sample simulations. It is interesting to note that for the longer maturities the breaks are dated earlier for the big EU member states France and Germany, whereas it is the opposite for the short maturities. An in-depth exploration of the economic or political reasons underlying these findings and break dates is beyond the purpose of the present paper where we merely intend to illustrate our procedure.

Remark 9. For financial data collected at higher frequencies, e.g. the considered daily CDS spreads series, the assumption of time-constant variances under the null hypothesis may be too restrictive. Similarly to observations made in e.g. Cavaliere and Taylor (2008), non-constant second moments of a form that lead to functional central limit theorems invoking time deformed Brownian motions - in this literature typically referred to as nonstationary volatility - will in general lead to asymptotic size distortions of our monitoring procedure, but will not invalidate consistency against fixed alternatives. ${ }^{21}$ To be precise, consistency of the detection procedure hinges on the fact that the longrun variance estimators computed over the pre-break period do not diverge in this more general setting.

\footnotetext{
${ }^{20}$ The strong nature of co-movements of series indicates that an extension of our monitoring procedures to monitor also structural change in the cross-sectional co-movements of potentially (co-)integrated series, in addition to changing (idiosyncratic) time series properties, may be relevant. Changes in the crosssectional dependence structure, in particular increasing dependencies in times of crisis, may be seen as an indicator of contagion phenomena.

${ }^{21}$ Cavaliere and Taylor (2005) consider the asymptotic behavior of the KPSS statistic in this context and Cavaliere and Taylor (2006) consider cointegration testing with variance breaks.
} 


\begin{tabular}{ccccc} 
Country & Maturity & Test statistic & $\tau_{m}(\alpha=0.1)$ & $\tau_{m}(\alpha=0.05)$ \\
\hline Austria & 1 & 4317.35 & 09.08 .2010 & 03.11 .2010 \\
& 3 & 4647.26 & 06.08 .2010 & 22.10 .2010 \\
& 5 & 5582.71 & 02.07 .2010 & 07.09 .2010 \\
& 7 & 5492.46 & 05.07 .2010 & 09.09 .2010 \\
Cyprus & 10 & 5643.04 & 28.06 .2010 & 02.09 .2010 \\
& 3 & 24474.22 & 07.07 .2010 & 10.09 .2010 \\
& 5 & 20564.81 & 16.07 .2010 & 24.09 .2010 \\
& 7 & 17693.05 & 30.06 .2010 & 02.09 .2010 \\
France & 10 & 16749.96 & 09.06 .2010 & 06.08 .2010 \\
& 3 & 3527.84 & 10.09 .2010 & 12.01 .2011 \\
& 5 & 12371.54 & 11.05 .2010 & 03.06 .2010 \\
& 7 & 14299.06 & 21.04 .2010 & 17.05 .2010 \\
& 10 & 16221.89 & 31.03 .2010 & 27.04 .2010 \\
\hline Germany & 1 & 3450.41 & 02.09 .2010 & 01.02 .2011 \\
& 3 & 3905.52 & 18.08 .2010 & 18.11 .2010 \\
& 5 & 7042.27 & 21.05 .2010 & 29.06 .2010 \\
& 7 & 8139.72 & 03.05 .2010 & 04.06 .2010 \\
& 10 & 9083.49 & 06.05 .2010 & 10.06 .2010 \\
\hline
\end{tabular}

Table 3: Results of stationarity monitoring for the daily CDS spreads data for $m=0.1$. The third column displays $\sup _{m \leq s \leq 1}\left|\frac{\hat{H}^{m}(s)}{g(s)}\right|$ and the fourth and fifth columns the associated detection times $\tau_{m}\left(\hat{H}^{m}(s), g(s), c(\alpha, w)\right)$ for $\alpha=\{0.1,0.05\}$. Intercept and linear trend are included, hence $g(s)=s^{5}$. The null hypothesis is rejected throughout. The $10 \%$ critical value is 1252.59 and the $5 \%$ critical value is 1777.80 . 


\section{Summary AND Conclusions}

We have proposed monitoring procedures for stationarity and cointegration that are based on parameter estimation on a pre-break calibration period. The key ingredients of our detectors are properly scaled squared partial sums of residuals that are compared between the calibration and the successively increasing monitoring period. Thus, our detectors are in the spirit of Chu et al. (1996) combined with the Kwiatkowski et al. (1992) respectively Shin (1994) tests. In case of cointegration monitoring we have investigated the properties of the monitoring procedure when using FM-OLS, D-OLS or IM-OLS residuals. Modified least squares estimation is necessary to correct for the effects of error serial correlation and regressor endogeneity in order to obtain nuisance parameter free limiting distributions. The procedures are shown to be consistent against I(1) alternatives, breaks in the parameters corresponding to the deterministic components and, in case of cointegration monitoring, breaks in the slope parameters.

The performance of the procedures has been investigated both in terms of local asymptotic power as well as by means of finite sample simulations. The effects of sample size, regressor endogeneity and error serial correlation are as typically found in the unit root and cointegration literature. Local asymptotic power and - in line with the asymptotic findings - finite sample (size corrected) power are in many configurations slightly lower for IM-OLS than for the other two procedures. However, IM-OLS has partly substantially lower size distortions under the null, which makes IM-OLS a good choice given that size corrections cannot be performed in actual applications. Detection in many circumstances occurs with delays, which are partly substantial. A longer calibration period is, as expected, beneficial in lessening the delays, as is a late break point.

A brief empirical application to CDS spreads data of four European countries indicates the usefulness of the proposed method for the case of stationarity monitoring. The break dates all precede the turbulent period of summer 2011 by between a year and half a year. Several extensions of the approach are conceivable: First, it may be relevant to flip null and alternative hypothesis, i.e. to monitor changes from $\mathrm{I}(1)$ to $\mathrm{I}(0)$ behavior (which 
could potentially be based on modifying our approach or by extending the approach of Steland and Weidauer, 2013). Second, the empirical application, with the clearly visible co-movements across maturities but also across countries, indicates that multivariate monitoring procedures may be important for applied research. Third, especially important for monitoring data collected at higher frequencies, the effects of non-constant variances need to be investigated in detail. Fourth, the impact of the weighting function on the performance of the monitoring procedures has to be investigated.

\section{REFERENCES}

Andrews, D. W. K. (1991): "Heteroskedasticity and Autocorrelation Consistent Covariance Matrix Estimation," Econometrica, 59, 817-854.

Aue, A., S. Hörmann, L. Horváth, M. Hušková, And J. Steinebach (2012): "Sequential Testing for the Stability of High Frequency Portfolio Betas," Econometric Theory, 28, 804-837.

Aue, A., L. Horváth, And M. L. Reimherr (2009): "Delay Times of Sequential Procedures for Multiple Time Series Regression Models," Journal of Econometrics, 149, 174-190.

Cappuccio, N. And D. Lubian (2005): "Local Asymptotic Distributions of Stationarity Tests," Journal of Time Series Analysis, 27, 323-345.

Cavaliere, G. And A. M. R. Taylor (2005): "Stationarity Tests under Time-Varying Second Moments," Econometric Theory, 21, 1112-1129.

— (2006): "Testing the Null of Cointegration in the Presence of Variance Breaks," Journal of Time Series Analysis, 27, 613-636.

- (2008): "Testing for a Change in Persistence in the Presence of Non-Stationary Volatility," Journal of Econometrics, 147, 84-98. 
Chen, Z., Z. Jin, Z. Tian, And P. Qi (2012): "Bootstrap Testing Multiple Changes in Persistence for a Heavy-Tailed Sequence," Computational Statistics and Data Analysis, $56,2303-2316$.

Chen, Z., Z. Tian, And Y. Wei (2010): "Monitoring Change in Persistence in Linear Time Series," Statistics \& Probability Letters, 80, 1520-1527.

Choi, I. And E. Kurozumi (2012): "Model Selection Criteria for the Leads-and-Lags Cointegrating Regression," Journal of Econometrics, 169, 224-238.

Chu, C.-S. J., M. Stinchcombe, And H. White (1996): "Monitoring Structural Change," Econometrica, 64, 1045-1065.

Dieckmann, S. And T. Plank (2011): "Default Risk of Advanced Economies: An Empirical Analysis of Credit Default Swaps During the Financial Crisis," Review of Finance, forthcoming, doi: 10.1093/rof/rfr015.

Groen, J. J. J., G. Kapetanios, and S. Price (2013): "Multivariate Methods for Monitoring Structural Change," Journal of Applied Econometrics, 28, 250-274.

Jansson, M. (2002): "Consistent Covariance Matrix Estimation for Linear Processes," Econometric Theory, 18, 1449-1459.

Kejriwal, M. And P. Perron (2008): "Data Dependent Rules for Selection of the Number of Leads and Lags in the Dynamic OLS Cointegrating Regressions," Econometric Theory, 24, 1425-1441.

KIM, J.-Y. (2000): "Detection of Change in Persistence of a Linear Time Series," Journal of Econometrics, 95, 97-116.

Kwiatkowski, D., P. C. B. Phillips, P. Schmidt, And Y. Shin (1992): "Testing the Null Hypothesis of Stationarity against the Alternative of a Unit Root," Journal of Econometrics, 54, 159-178. 
Phillips, P. C. B. (1987): "Towards a Unified Asymptotic Theory for Autoregression," Biometrika, 74, 535-547.

Phillips, P. C. B. And B. Hansen (1990): "Statistical Inference in Instrumental Variables Regression with I(1) Processes," Review of Economic Studies, 57, 99-125.

Phillips, P. C. B. And M. Loretan (1993): "Estimating Long Run Economic Equilibria," Review of Economic Studies, 58, 407-436.

Phillips, P. C. B., Y. Wu, And J. Yu (2011): "Explosive Behavior in the 1990s Nasdaq: When did Exuberance Escalate Asset Values?" International Economic Review, $52,201-226$.

Qi, P., Z. Jin, Z. Tian, And Z. Chen (2013): "Monitoring Persistent Change in a Heavy-Tailed Sequence with Polynomial Trends," Journal of the Korean Statistical Society, 42, 497-506.

SAIKKonen, P. (1991): “Asymptotically Efficient Estimation of Cointegrating Regressions," Econometric Theory, 7, 1-21.

SHIN, Y. (1994): "A Residual-Based Test of the Null of Cointegration against the Alternative of No Cointegration," Econometric Theory, 10, 91-115.

Steland, A. (2007): "Monitoring Procedures to Detect Unit Roots and Stationarity," Econometric Theory, 23, 1108-1135.

(2008): "Sequentially Updated Residuals and Detection of Stationary Errors in Polynomial Regression Models," Sequential Analysis: Design Methods and Applications, 27, 304-329.

Steland, A. And S. Weidauer (2013): "Detection of Stationary Errors in Multiple Regressions with Integrated Regressors and Cointegration," Sequential Analysis, 32, $319-349$. 
Stock, J. H. AND M. W. Watson (1993): "A Simple Estimator of Cointegrating Vectors in Higher Order Integrated Systems," Econometrica, 61, 783-820.

Vogelsang, T. J. And M. Wagner (2014): "Integrated Modified OLS Estimation and Fixed-b Inference for Cointegrating Regressions," Journal of Econometrics, 148, $741-760$.

Wied, D. And P. Galeano (2013): "Monitoring Correlation Change in a Sequence of Random Variables," Journal of Statistical Planning and Inference, 143, 186-196. 


\section{A. Proofs}

Proof of Lemma 1:

The result follows directly from the assumptions using consistency of OLS detrending (used already for the FCLT in (13)) and of long-run variance estimation.

\section{Proof of Proposition 1:}

The result follows from Lemma 1 since the limit of $\widehat{H}^{m}(s), \widehat{\mathcal{H}}^{m}(s)$ is well defined and the continuous mapping theorem, compare (11) with $\widehat{H}^{m}(s)$ instead of $H^{m}(s)$. Proof of Proposition 2:

(a): Start by decomposing the partial sum process, for $1 \geq s>r \geq m$ into

$$
\frac{1}{\sqrt{T}} \widehat{S}_{[s T]}=\frac{1}{\sqrt{T}} \widehat{S}_{[r T]}+\frac{1}{\sqrt{T}} \sum_{t=[r T]+1}^{[s T]} \hat{u}_{t, m}
$$

The first term above converges to $\omega \widehat{W}_{m}(r)$ and the second term above is unbounded under the considered alternative, since

$$
\frac{1}{\sqrt{T}} \sum_{t=[r T]+1}^{[s T]} \hat{u}_{t, m}=\frac{1}{\sqrt{T}} \sum_{t=[r T]+1}^{[s T]} u_{t}-\frac{1}{\sqrt{T}} \sum_{t=[r T]+1}^{[s T]} D_{t}^{\prime}\left(\sum_{i=1}^{[m T]} D_{i} D_{i}^{\prime}\right)^{-1} \sum_{i=1}^{[m T]} D_{i} u_{i}
$$

where the first term is $O_{p}(T)$, as it converges, when scaled by $T^{-1}$, towards the integral of a Brownian motion, and the second term converges to $\int_{r}^{s} D(z) d z^{\prime}\left(\int_{0}^{m} D(z) D(z)^{\prime} d z\right)^{-1}$ $\int_{0}^{m} D(z) d W(z)$. Consequently, for $s>r$, the first term in $\widehat{H}^{m}(s)$ and thus $\widehat{H}^{m}(s)$ diverges, which establishes the result.

(b): Straightforward calculations similar to the one above establish for $1 \geq s>r \geq m$ that 


$$
\begin{aligned}
\frac{1}{\sqrt{T}} \sum_{t=1}^{[s T]} \hat{u}_{t, m} & =\frac{1}{\sqrt{T}} \sum_{t=1}^{[s T]} u_{t}-\frac{1}{\sqrt{T}} \sum_{t=1}^{[s T]} D_{t}^{\prime}\left(\sum_{i=1}^{[m T]} D_{i} D_{i}^{\prime}\right)^{-1} \sum_{i=1}^{[m T]} D_{i} u_{i} \\
& =\frac{1}{\sqrt{T}} \sum_{t=1}^{[s T]} u_{t}^{0}+\frac{1}{\sqrt{T}} \sum_{t=1}^{[s T]} \frac{\delta}{T} \sum_{i=[r T]+1}^{t} \xi_{i}-\frac{1}{\sqrt{T}} \sum_{t=1}^{[s T]} D_{t}^{\prime}\left(\sum_{i=1}^{[m T]} D_{i} D_{i}^{\prime}\right)^{-1} \sum_{i=1}^{[m T]} D_{i} u_{i}^{0} \\
& \Rightarrow \omega \widehat{W}_{m}(s)+\delta \omega_{\xi} \int_{r}^{s}\left(W_{\xi}(z)-W_{\xi}(r)\right) d z .
\end{aligned}
$$

This shows that the partial sum process can be made arbitrarily large (with arbitrarily large probability) by choosing $\delta$ large enough. This in turn makes the probability of a finite $\tau_{m}$ arbitrarily close to one.

\section{Proof of Proposition 3:}

(a): Again the starting point is the partial sum process of the OLS residuals. Consider again $1 \geq s>r \geq m$ :

$\frac{1}{\sqrt{T}} \sum_{t=1}^{[s T]} \hat{u}_{t, m}=\frac{1}{\sqrt{T}} \sum_{t=1}^{[s T]} u_{t}-\frac{1}{\sqrt{T}} \sum_{t=1}^{[s T]} D_{t}^{\prime}\left(\sum_{i=1}^{[m T]} D_{i} D_{i}^{\prime}\right)^{-1} \sum_{i=1}^{[m T]} D_{i} u_{i}-\frac{1}{\sqrt{T}} \sum_{t=[r T]+1}^{[s T]} D_{t}^{\prime}\left(\theta_{D, 1}-\theta_{D, 2}\right)$.

The first two terms above converge to $\omega \widehat{W}_{m}(s)$ and the third term diverges due to Assumption $1 .^{22}$

(b): When considering local alternatives, the partial sum process is as follows (for $1 \geq$ $s>r \geq m)$ :

\footnotetext{
${ }^{22}$ To be precise, divergence occurs for all trend functions such that $G_{D}$ diverges.
} 


$$
\begin{aligned}
\frac{1}{\sqrt{T}} \sum_{t=1}^{[s T]} \hat{u}_{t, m} & =\frac{1}{\sqrt{T}} \sum_{t=1}^{[s T]} u_{t} \\
& -\frac{1}{\sqrt{T}} \sum_{t=1}^{[s T]} D_{t}^{\prime}\left(\sum_{i=1}^{[m T]} D_{i} D_{i}^{\prime}\right)^{-1} \sum_{i=1}^{[m T]} D_{i} u_{i} \\
& +\frac{1}{\sqrt{T}} \sum_{t=[r T]+1}^{[s T]} D_{t}^{\prime} G_{D}^{-1 \prime} \Delta_{\theta} .
\end{aligned}
$$

Under the stated assumptions the limit is given by

$$
\frac{1}{\sqrt{T}} \sum_{t=1}^{[s T]} \hat{u}_{t, m} \Rightarrow \omega \widehat{W}_{m}(s)+\int_{r}^{s} D(z)^{\prime} d z \Delta_{\theta}
$$

from which the result follows.

Proof of Lemmata 2, 3, 4 and 5:

These lemmata all follow immediately from the convergence properties of the underlying estimation methods in conjunction with consistent long-run variance estimation and the continuous mapping theorem.

\section{Proof of Proposition 4:}

The argument is similar to the argument used in Proposition 1 and follows from the FCLT provided in Vogelsang and Wagner (2014, Lemma 2).

\section{Proof of Proposition 5:}

(a): Again, the limiting behavior of the partial sum process of the residuals is the key to the result, where we now have to distinguish between two cases, FM-OLS and D-OLS estimation on the one hand and IM-OLS estimation on the other. Again we outline the arguments here for the FM-OLS estimator, noting that the limit process is similar for D-OLS estimation, and the IM-OLS estimator. 
For FM-OLS, the partial sum process of the residuals is given by (again for $1 \geq s>r \geq$ $m):$

$$
\begin{aligned}
\frac{1}{\sqrt{T}} \sum_{t=1}^{[s T]} \hat{u}_{t, m}^{+}= & \frac{1}{\sqrt{T}} \sum_{t=1}^{[r T]} \hat{u}_{t, m}^{+}+\frac{1}{\sqrt{T}} \sum_{t=[r T]+1}^{[s T]} \hat{u}_{t, m}^{+} \\
= & \frac{1}{\sqrt{T}} \sum_{t=1}^{[r T]} \hat{u}_{t, m}^{+}+\frac{1}{\sqrt{T}} \sum_{t=[r T]+1}^{[s T]} u_{t}-\frac{1}{\sqrt{T}} \sum_{t=[r T]+1}^{[s T]} v_{t}^{\prime} \hat{\Omega}_{v v}^{-1} \hat{\Omega}_{v u}- \\
& -\frac{1}{\sqrt{T}} \sum_{t=[r T]+1}^{[s T]} D_{t}^{\prime}\left(\hat{\theta}_{D, m}-\theta_{D}\right)-\frac{1}{\sqrt{T}} \sum_{t=[r T]+1}^{[s T]} X_{t}^{\prime}\left(\hat{\theta}_{X, m}-\theta_{X}\right) .
\end{aligned}
$$

The first term above converges to $\omega_{u \cdot v} \widehat{W}_{u \cdot v}(r)$, according to Lemma 2 and the second term diverges since for the sample period considered $u_{t}$ is an $\mathrm{I}(1)$ process. The remaining three terms converge in distribution. Thus, the partial sum process is in this case $O_{p}(T)$. The argument is analogous for the IM-OLS partial sum process, i.e. for $\hat{S}_{t, m}^{u}$, with Lemma 4 replacing Lemma 2.

(b): Also for this result similar arguments as in Proposition 2 apply. In particular it follows now for FM-OLS that (for $1 \geq s>r \geq m$ ):

$$
\frac{1}{\sqrt{T}} \sum_{t=1}^{[s T]} \hat{u}_{t, m}^{+} \Rightarrow \omega_{u \cdot v} \widehat{W}_{u \cdot v}(s)+\delta \omega_{\xi} \int_{r}^{s}\left(W_{\xi}(z)-W_{\xi}(r)\right) d z .
$$

Thus, the same argument as in Proposition 2 applies. For IM-OLS the result is of the same type and given by

$$
\frac{1}{\sqrt{T}} \hat{S}_{[s T], m}^{u} \Rightarrow \omega_{u \cdot v} \tilde{P}_{m}(s)+\delta \omega_{\xi} \int_{r}^{s}\left(W_{\xi}(z)-W_{\xi}(r)\right) d z
$$

The results follow, since the identical second term can be again made arbitrarily large by choice of $\delta$ for both cases.

\section{Proof of Proposition 6:}

The arguments are similar to the arguments in Proposition 3, with the only difference 
being the exact form of the terms, due to the presence of integrated regressors $X_{t}$. For brevity we again just look at the FM-OLS expressions and note again that the result for D-OLS coincides asymptotically.

(a): Under the fixed alternative, the partial sum process of the FM-OLS residuals, again for $1 \geq s>r \geq m$, can be written as:

$$
\begin{aligned}
\frac{1}{\sqrt{T}} \sum_{t=1}^{[s T]} \hat{u}_{t, m}^{+} & =\frac{1}{\sqrt{T}} \sum_{t=1}^{[s T]} u_{t}-\frac{1}{\sqrt{T}} \sum_{t=1}^{[s T]} v_{t}^{\prime} \hat{\Omega}_{v v}^{-1} \hat{\Omega}_{v u} \\
& -\frac{1}{\sqrt{T}} \sum_{t=1}^{[s T]} D_{t}^{\prime}\left(\hat{\theta}_{D, m}-\theta_{D, 1}\right)-\frac{1}{\sqrt{T}} \sum_{t=1}^{[s T]} X_{t}^{\prime}\left(\hat{\theta}_{X, m}-\theta_{X}\right) \\
& -\frac{1}{\sqrt{T}} \sum_{t=[r T]+1}^{[s T]} D_{t}^{\prime}\left(\theta_{D, 1}-\theta_{D, 2}\right) .
\end{aligned}
$$

From this expression it is seen that the result is similar to the result in Proposition 3, since the last term diverges and all other terms together converge to $\omega_{u \cdot v} \widehat{W}_{u \cdot v}(s)$.

(b): Also here the result is analogous, with the only difference being that under local alternatives the last term in (62) changes, as in part (b) of Proposition 3, and is instead given by

$$
\frac{1}{\sqrt{T}} \sum_{t=[r T]+1}^{[s T]} D_{t}^{\prime} G_{D}^{-1 \prime} \Delta_{\theta} \Rightarrow \int_{r}^{s} D(z)^{\prime} d z \Delta_{\theta}
$$

This shows the result as under the considered local alternatives it holds that

$$
\frac{1}{\sqrt{T}} \sum_{t=1}^{[s T]} u_{t, m}^{+} \Rightarrow \omega_{u \cdot v} W_{u \cdot v}(s)+\int_{r}^{s} D(z)^{\prime} d z \Delta_{\theta} .
$$

The arguments are analogous for IM-OLS with the corresponding limit of the residual process given by

$$
\frac{1}{\sqrt{T}} \hat{S}_{[s T], m}^{u} \Rightarrow \omega_{u \cdot v} \tilde{P}_{m}(s)+\int_{r}^{s} D(z)^{\prime} d z \Delta_{\theta}
$$




\section{Proof of Proposition \%:}

The proof is analogous to the proof of Proposition 5 with the changes following from the discussion before Proposition 7.

(a): For FM-OLS we get for $1 \geq s>r \geq m$ :

$$
\begin{aligned}
\frac{1}{\sqrt{T}} \sum_{t=1}^{[s T]} \hat{u}_{t, m}^{+} & =\frac{1}{\sqrt{T}} \sum_{t=1}^{[s T]} u_{t}-\frac{1}{\sqrt{T}} \sum_{t=1}^{[s T]} v_{t}^{\prime} \hat{\Omega}_{v v}^{-1} \hat{\Omega}_{v u} \\
& -\frac{1}{\sqrt{T}} \sum_{t=1}^{[s T]} D_{t}^{\prime}\left(\hat{\theta}_{D, m}-\theta_{D}\right)-\frac{1}{\sqrt{T}} \sum_{t=1}^{[s T]} X_{t}^{\prime}\left(\hat{\theta}_{X, m}-\theta_{X, 1}\right) \\
& -\frac{1}{\sqrt{T}} \sum_{t=[r T]+1}^{[s T]} X_{t}^{\prime}\left(\theta_{X, 1}-\theta_{X, 2}\right) .
\end{aligned}
$$

The first four terms together converge to $\omega_{u \cdot v} \widehat{W}_{u \cdot v}(s)$ and the last term is $O_{p}(T)$. For IM-OLS we get for $1 \geq s>r \geq m$ :

$$
\begin{aligned}
\frac{1}{\sqrt{T}} \hat{S}_{[s T], m}^{u} & =\frac{1}{\sqrt{T}} S_{[s T]}^{u}-\frac{1}{\sqrt{T}} S_{[s T]}^{D \prime}\left(\hat{\theta}_{D, m}-\theta_{D}\right)-\frac{1}{\sqrt{T}} S_{[s T]}^{X \prime}\left(\hat{\theta}_{X, m}-\theta_{X, 1}\right) \\
& -\frac{1}{\sqrt{T}} X_{[s T]}^{\prime} \hat{\varphi}_{m}-\frac{1}{\sqrt{T}} S_{[s T]}^{X \prime}\left(\theta_{X, 1}-\theta_{X, 2}\right)
\end{aligned}
$$

from which it follows that the first four terms together converge to $\omega_{u \cdot v} \tilde{P}_{m}(s)$ and the last term is $O_{p}(T)$.

(b): The changes implied by the local alternatives considered are similar to the previous propositions and we obtain for FM-OLS for $1 \geq s>r \geq m$ : 


$$
\begin{aligned}
\frac{1}{\sqrt{T}} \sum_{t=1}^{[s T]} \hat{u}_{t, m}^{+} & =\frac{1}{\sqrt{T}} \sum_{t=1}^{[s T]} u_{t}-\frac{1}{\sqrt{T}} \sum_{t=1}^{[s T]} v_{t}^{\prime} \hat{\Omega}_{v v}^{-1} \hat{\Omega}_{v u} \\
& -\frac{1}{\sqrt{T}} \sum_{t=1}^{[s T]} D_{t}^{\prime}\left(\hat{\theta}_{D, m}-\theta_{D, 1}\right)-\frac{1}{\sqrt{T}} \sum_{t=1}^{[s T]} X_{t}^{\prime}\left(\hat{\theta}_{X, m}-\theta_{X}\right) \\
& +\frac{1}{T^{3 / 2}} \sum_{t=[r T]+1}^{[s T]} X_{t}^{\prime} \Delta_{\theta} \\
& \Rightarrow \omega_{u \cdot v} \widehat{W}_{u \cdot v}(s)+\int_{r}^{s} W_{v}(z)^{\prime} d z \Omega_{v v}^{1 / 2 \prime} \Delta_{\theta} .
\end{aligned}
$$

The result for IM-OLS follows analogously to the result for FM-OLS from the result in part (a) and we obtain for $1 \geq s>r \geq m$ :

$$
\frac{1}{\sqrt{T}} \hat{S}_{[s T], m}^{u} \Rightarrow \omega_{u \cdot v} \tilde{P}_{m}(s)+\int_{0}^{s} W_{v}(z)^{\prime} d z \Omega_{v v}^{1 / 2 \prime} \Delta_{\theta}
$$


B. Critical Values 


\begin{tabular}{|c|c|c|c|c|c|c|c|c|c|}
\hline$m$ & $90 \%$ & $95 \%$ & $97.5 \%$ & $99 \%$ & $m$ & $90 \%$ & $95 \%$ & $97.5 \%$ & $99 \%$ \\
\hline 0.10 & 8.41 & 11.37 & 14.54 & 18.94 & 0.51 & 0.79 & 1.01 & 1.24 & 1.55 \\
\hline 0.11 & 7.50 & 10.10 & 12.94 & 16.84 & 0.52 & 0.76 & 0.98 & 1.20 & 1.51 \\
\hline 0.12 & 6.73 & 9.08 & 11.61 & 15.09 & 0.53 & 0.74 & 0.95 & 1.17 & 1.46 \\
\hline 0.13 & 6.08 & 8.21 & 10.46 & 13.62 & 0.54 & 0.72 & 0.92 & 1.13 & 1.42 \\
\hline 0.14 & 5.54 & 7.45 & 9.50 & 12.36 & 0.55 & 0.69 & 0.90 & 1.10 & 1.38 \\
\hline 0.15 & 5.06 & 6.81 & 8.66 & 11.29 & 0.56 & 0.67 & 0.87 & 1.08 & 1.35 \\
\hline 0.16 & 4.65 & 6.24 & 7.94 & 10.33 & 0.57 & 0.66 & 0.85 & 1.05 & 1.32 \\
\hline 0.17 & 4.29 & 5.76 & 7.34 & 9.53 & 0.58 & 0.64 & 0.83 & 1.02 & 1.29 \\
\hline 0.18 & 3.98 & 5.33 & 6.77 & 8.80 & 0.59 & 0.62 & 0.81 & 1.00 & 1.27 \\
\hline 0.19 & 3.70 & 4.94 & 6.28 & 8.15 & 0.60 & 0.61 & 0.79 & 0.98 & 1.24 \\
\hline 0.20 & 3.44 & 4.59 & 5.83 & 7.56 & 0.61 & 0.59 & 0.77 & 0.96 & 1.22 \\
\hline 0.21 & 3.22 & 4.29 & 5.44 & 7.04 & 0.62 & 0.58 & 0.76 & 0.94 & 1.20 \\
\hline 0.22 & 3.01 & 4.01 & 5.07 & 6.56 & 0.63 & 0.56 & 0.74 & 0.92 & 1.18 \\
\hline 0.23 & 2.83 & 3.76 & 4.74 & 6.13 & 0.64 & 0.55 & 0.73 & 0.91 & 1.15 \\
\hline 0.24 & 2.66 & 3.52 & 4.44 & 5.73 & 0.65 & 0.54 & 0.71 & 0.89 & 1.14 \\
\hline 0.25 & 2.50 & 3.31 & 4.17 & 5.37 & 0.66 & 0.53 & 0.70 & 0.88 & 1.12 \\
\hline 0.26 & 2.36 & 3.11 & 3.92 & 5.05 & 0.67 & 0.52 & 0.69 & 0.86 & 1.10 \\
\hline 0.27 & 2.23 & 2.94 & 3.69 & 4.75 & 0.68 & 0.51 & 0.68 & 0.85 & 1.09 \\
\hline 0.28 & 2.11 & 2.78 & 3.48 & 4.48 & 0.69 & 0.50 & 0.67 & 0.84 & 1.07 \\
\hline 0.29 & 2.00 & 2.63 & 3.29 & 4.22 & 0.70 & 0.50 & 0.66 & 0.83 & 1.05 \\
\hline 0.30 & 1.90 & 2.49 & 3.11 & 3.98 & 0.71 & 0.49 & 0.65 & 0.81 & 1.04 \\
\hline 0.31 & 1.81 & 2.36 & 2.94 & 3.76 & 0.72 & 0.48 & 0.64 & 0.80 & 1.02 \\
\hline 0.32 & 1.72 & 2.24 & 2.79 & 3.56 & 0.73 & 0.47 & 0.63 & 0.79 & 1.01 \\
\hline 0.33 & 1.64 & 2.13 & 2.65 & 3.37 & 0.74 & 0.47 & 0.62 & 0.78 & 1.00 \\
\hline 0.34 & 1.56 & 2.03 & 2.52 & 3.20 & 0.75 & 0.46 & 0.61 & 0.77 & 0.99 \\
\hline 0.35 & 1.49 & 1.93 & 2.40 & 3.03 & 0.76 & 0.45 & 0.60 & 0.76 & 0.97 \\
\hline 0.36 & 1.42 & 1.85 & 2.28 & 2.89 & 0.77 & 0.45 & 0.60 & 0.75 & 0.96 \\
\hline 0.37 & 1.36 & 1.76 & 2.18 & 2.75 & 0.78 & 0.44 & 0.59 & 0.74 & 0.95 \\
\hline 0.38 & 1.30 & 1.68 & 2.08 & 2.62 & 0.79 & 0.44 & 0.58 & 0.73 & 0.94 \\
\hline 0.39 & 1.25 & 1.61 & 1.9 & 2.49 & 0.80 & 0.43 & 0.57 & 0.72 & 0.92 \\
\hline 0.40 & 1.19 & 1.54 & 1.90 & 2.38 & 0.81 & 0.43 & 0.57 & 0.71 & 0.91 \\
\hline 0.41 & 1.15 & 1.47 & 1.82 & 2.27 & 0.82 & 0.42 & 0.56 & 0.70 & 0.90 \\
\hline 0.42 & 1.10 & 1.42 & 1.74 & 2.18 & 0.83 & 0.42 & 0.55 & 0.70 & 0.89 \\
\hline 0.43 & 1.06 & 1.36 & 1.67 & 2.09 & 0.84 & 0.41 & 0.55 & 0.69 & 0.88 \\
\hline 0.44 & 1.02 & 1.30 & 1.60 & 2.00 & 0.85 & 0.41 & 0.54 & 0.68 & 0.87 \\
\hline 0.45 & 0.98 & 1.25 & 1.54 & 1.92 & 0.86 & 0.40 & 0.54 & 0.67 & 0.86 \\
\hline 0.46 & 0.94 & 1.21 & 1.48 & 1.85 & 0.87 & 0.40 & 0.53 & 0.66 & 0.85 \\
\hline 0.47 & 0.91 & 1.16 & 1.43 & 1.78 & 0.88 & 0.39 & 0.52 & 0.66 & 0.84 \\
\hline 0.48 & 0.87 & 1.12 & 1.38 & 1.72 & 0.89 & 0.39 & 0.52 & 0.65 & 0.83 \\
\hline 0.49 & 0.85 & 1.09 & 1.33 & 1.66 & 0.90 & 0.39 & 0.51 & 0.64 & 0.82 \\
\hline 0.50 & 0.82 & 1.05 & 1.28 & 1.60 & & & & & \\
\hline
\end{tabular}

Table 4: Critical values for stationarity monitoring for the intercept case. 


\begin{tabular}{|c|c|c|c|c|c|c|c|c|c|}
\hline$m$ & $90 \%$ & $95 \%$ & $97.5 \%$ & $99 \%$ & $m$ & $90 \%$ & $95 \%$ & $97.5 \%$ & $99 \%$ \\
\hline 0.10 & 1252.59 & 1777.80 & 2327.20 & 3066.19 & 0.51 & 2.36 & 3.32 & 4.33 & 5.73 \\
\hline 0.11 & 916.45 & 1300.66 & 1702.68 & 2242.99 & 0.52 & 2.13 & 2.99 & 3.90 & 5.15 \\
\hline 0.12 & 685.24 & 977.06 & 1277.92 & 1684.26 & 0.53 & 1.92 & 2.70 & 3.52 & 4.64 \\
\hline 0.13 & 526.01 & 748.01 & 978.33 & 1292.92 & 0.54 & 1.74 & 2.43 & 3.18 & 4.19 \\
\hline 0.14 & 409.41 & 580.95 & 760.56 & 1003.24 & 0.55 & 1.58 & 2.20 & 2.86 & 3.78 \\
\hline 0.15 & 323.20 & 459.38 & 599.14 & 792.11 & 0.56 & 1.43 & 1.99 & 2.59 & 3.42 \\
\hline 0.16 & 259.06 & 366.74 & 480.88 & 634.05 & 0.57 & 1.30 & 1.79 & 2.33 & 3.08 \\
\hline 0.17 & 209.66 & 297.55 & 388.27 & 512.91 & 0.58 & 1.18 & 1.62 & 2.11 & 2.77 \\
\hline 0.18 & 171.47 & 243.49 & 318.84 & 421.52 & 0.59 & 1.07 & 1.47 & 1.90 & 2.50 \\
\hline 0.19 & 141.61 & 200.86 & 263.26 & 347.20 & 0.60 & 0.98 & 1.32 & 1.71 & 2.25 \\
\hline 0.20 & 117.97 & 167.16 & 218.50 & 287.23 & 0.61 & 0.89 & 1.20 & 1.55 & 2.04 \\
\hline 0.21 & 99.05 & 140.26 & 183.14 & 241.95 & 0.62 & 0.81 & 1.09 & 1.40 & 1.83 \\
\hline 0.22 & 83.56 & 118.52 & 155.38 & 204.50 & 0.63 & 0.74 & 0.99 & 1.26 & 1.66 \\
\hline 0.23 & 71.00 & 100.76 & 131.56 & 173.22 & 0.64 & 0.68 & 0.90 & 1.14 & 1.49 \\
\hline 0.24 & 60.64 & 85.92 & 112.21 & 148.82 & 0.65 & 0.63 & 0.82 & 1.03 & 1.34 \\
\hline 0.25 & 51.96 & 73.73 & 96.37 & 126.62 & 0.66 & 0.58 & 0.75 & 0.94 & 1.21 \\
\hline 0.26 & 44.71 & 63.46 & 82.84 & 109.29 & 0.67 & 0.53 & 0.68 & 0.85 & 1.09 \\
\hline 0.27 & 38.77 & 55.02 & 71.90 & 94.71 & 0.68 & 0.49 & 0.63 & 0.77 & 0.98 \\
\hline 0.28 & 33.62 & 47.80 & 62.36 & 82.22 & 0.69 & 0.46 & 0.58 & 0.71 & 0.89 \\
\hline 0.29 & 29.32 & 41.64 & 54.36 & 71.81 & 0.70 & 0.42 & 0.53 & 0.65 & 0.81 \\
\hline 0.30 & 25.61 & 36.39 & 47.56 & 62.62 & 0.71 & 0.40 & 0.49 & 0.60 & 0.74 \\
\hline 0.31 & 22.45 & 31.85 & 41.67 & 54.92 & 0.72 & 0.37 & 0.46 & 0.55 & 0.68 \\
\hline 0.32 & 19.74 & 27.97 & 36.70 & 48.32 & 0.73 & 0.35 & 0.43 & 0.51 & 0.63 \\
\hline 0.33 & 17.42 & 24.64 & 32.24 & 42.61 & 0.74 & 0.32 & 0.40 & 0.48 & 0.58 \\
\hline 0.34 & 15.36 & 21.83 & 28.51 & 37.57 & 0.75 & 0.30 & 0.38 & 0.45 & 0.55 \\
\hline 0.35 & 13.60 & 19.30 & 25.22 & 33.24 & 0.76 & 0.29 & 0.35 & 0.42 & 0.51 \\
\hline 0.36 & 12.09 & 17.13 & 22.40 & 29.55 & 0.77 & 0.27 & 0.34 & 0.40 & 0.49 \\
\hline 0.37 & 10.73 & 15.21 & 19.88 & 26.23 & 0.78 & 0.26 & 0.32 & 0.38 & 0.46 \\
\hline 0.38 & 9.57 & 13.54 & 17.68 & 23.33 & 0.79 & 0.25 & 0.30 & 0.36 & 0.44 \\
\hline 0.39 & 8.53 & 12.07 & 15.79 & 20.76 & 0.80 & 0.23 & 0.29 & 0.35 & 0.42 \\
\hline 0.40 & 7.61 & 10.77 & 14.08 & 18.56 & 0.81 & 0.22 & 0.28 & 0.33 & 0.41 \\
\hline 0.41 & 6.80 & 9.61 & 12.57 & 16.63 & 0.82 & 0.22 & 0.27 & 0.32 & 0.39 \\
\hline 0.42 & 6.09 & 8.61 & 11.25 & 14.92 & 0.83 & 0.21 & 0.26 & 0.31 & 0.38 \\
\hline 0.43 & 5.46 & 7.75 & 10.09 & 13.32 & 0.84 & 0.20 & 0.25 & 0.30 & 0.36 \\
\hline 0.44 & 4.91 & 6.96 & 9.06 & 11.96 & 0.85 & 0.19 & 0.24 & 0.29 & 0.35 \\
\hline 0.45 & 4.41 & 6.24 & 8.13 & 10.71 & 0.86 & 0.19 & 0.23 & 0.28 & 0.34 \\
\hline 0.46 & 3.97 & 5.61 & 7.33 & 9.67 & 0.87 & 0.18 & 0.22 & 0.27 & 0.33 \\
\hline 0.47 & 3.57 & 5.05 & 6.61 & 8.73 & 0.88 & 0.17 & 0.22 & 0.26 & 0.32 \\
\hline 0.48 & 3.21 & 4.55 & 5.96 & 7.87 & 0.89 & 0.17 & 0.21 & 0.25 & 0.31 \\
\hline 0.49 & 2.90 & 4.10 & 5.36 & 7.04 & 0.90 & 0.16 & 0.20 & 0.24 & 0.30 \\
\hline 0.50 & 2.61 & 3.70 & 4.81 & 6.34 & & & & & \\
\hline
\end{tabular}

Table 5: Critical values for stationarity monitoring for the intercept and linear trend case. 


\begin{tabular}{|c|c|c|c|c|c|c|c|c|c|}
\hline$m$ & $90 \%$ & $95 \%$ & $97.5 \%$ & $99 \%$ & $m$ & $90 \%$ & $95 \%$ & $97.5 \%$ & $99 \%$ \\
\hline 0.10 & 155.37 & 296.84 & 502.54 & 902.43 & 0.51 & 1.33 & 2.14 & 3.25 & 5.25 \\
\hline 0.11 & 124.53 & 238.91 & 403.91 & 723.99 & 0.52 & 1.22 & 1.96 & 2.97 & 4.76 \\
\hline 0.12 & 101.35 & 193.34 & 330.54 & 589.85 & 0.53 & 1.13 & 1.79 & 2.70 & 4.31 \\
\hline 0.13 & 83.57 & 159.61 & 269.82 & 483.63 & 0.54 & 1.05 & 1.63 & 2.45 & 3.90 \\
\hline 0.14 & 69.99 & 133.19 & 224.75 & 401.60 & 0.55 & 0.97 & 1.50 & 2.23 & 3.53 \\
\hline 0.15 & 58.93 & 111.90 & 188.55 & 333.77 & 0.56 & 0.91 & 1.37 & 2.03 & 3.19 \\
\hline 0.16 & 50.22 & 94.76 & 160.22 & 283.59 & 0.57 & 0.84 & 1.26 & 1.84 & 2.87 \\
\hline 0.17 & 42.90 & 81.12 & 136.42 & 240.58 & 0.58 & 0.79 & 1.16 & 1.67 & 2.62 \\
\hline 0.18 & 36.94 & 69.60 & 117.13 & 207.64 & 0.59 & 0.73 & 1.07 & 1.53 & 2.35 \\
\hline 0.19 & 32.13 & 60.17 & 101.28 & 178.28 & 0.60 & 0.69 & 0.99 & 1.40 & 2.14 \\
\hline 0.20 & 28.06 & 52.42 & 87.90 & 154.94 & 0.61 & 0.64 & 0.92 & 1.28 & 1.93 \\
\hline 0.21 & 24.62 & 45.98 & 76.70 & 134.76 & 0.62 & 0.61 & 0.86 & 1.18 & 1.74 \\
\hline 0.22 & 21.67 & 40.30 & 67.25 & 118.09 & 0.63 & 0.57 & 0.80 & 1.08 & 1.59 \\
\hline 0.23 & 19.16 & 35.53 & 59.29 & 103.42 & 0.64 & 0.54 & 0.75 & 1.00 & 1.45 \\
\hline 0.24 & 17.00 & 31.43 & 51.89 & 90.86 & 0.65 & 0.51 & 0.70 & 0.93 & 1.33 \\
\hline 0.25 & 15.13 & 27.87 & 46.01 & 79.96 & 0.66 & 0.48 & 0.66 & 0.87 & 1.21 \\
\hline 0.26 & 13.49 & 24.75 & 40.78 & 70.51 & 0.67 & 0.46 & 0.62 & 0.81 & 1.12 \\
\hline 0.27 & 12.10 & 22.17 & 36.50 & 63.01 & 0.68 & 0.43 & 0.59 & 0.77 & 1.05 \\
\hline 0.28 & 10.87 & 19.82 & 32.65 & 56.42 & 0.69 & 0.41 & 0.56 & 0.72 & 0.97 \\
\hline 0.29 & 9.78 & 17.75 & 29.23 & 50.12 & 0.70 & 0.40 & 0.53 & 0.69 & 0.92 \\
\hline 0.30 & 8.81 & 15.99 & 26.18 & 44.75 & 0.71 & 0.38 & 0.51 & 0.66 & 0.87 \\
\hline 0.31 & 7.98 & 14.39 & 23.45 & 40.20 & 0.72 & 0.36 & 0.49 & 0.63 & 0.83 \\
\hline 0.32 & 7.21 & 12.98 & 21.05 & 36.15 & 0.73 & 0.35 & 0.47 & 0.61 & 0.80 \\
\hline 0.33 & 6.51 & 11.68 & 18.94 & 32.28 & 0.74 & 0.34 & 0.46 & 0.58 & 0.77 \\
\hline 0.34 & 5.91 & 10.55 & 17.09 & 29.00 & 0.75 & 0.33 & 0.44 & 0.57 & 0.75 \\
\hline 0.35 & 5.37 & 9.54 & 15.39 & 26.04 & 0.76 & 0.32 & 0.43 & 0.55 & 0.73 \\
\hline 0.36 & 4.88 & 8.65 & 13.87 & 23.28 & 0.77 & 0.31 & 0.42 & 0.54 & 0.71 \\
\hline 0.37 & 4.45 & 7.83 & 12.57 & 21.05 & 0.78 & 0.31 & 0.41 & 0.53 & 0.70 \\
\hline 0.38 & 4.06 & 7.08 & 11.37 & 19.04 & 0.79 & 0.30 & 0.40 & 0.52 & 0.69 \\
\hline 0.39 & 3.70 & 6.45 & 10.30 & 17.28 & 0.80 & 0.29 & 0.40 & 0.51 & 0.68 \\
\hline 0.40 & 3.38 & 5.85 & 9.31 & 15.62 & 0.81 & 0.29 & 0.39 & 0.51 & 0.67 \\
\hline 0.41 & 3.10 & 5.33 & 8.44 & 14.15 & 0.82 & 0.29 & 0.39 & 0.50 & 0.66 \\
\hline 0.42 & 2.82 & 4.85 & 7.69 & 12.79 & 0.83 & 0.28 & 0.38 & 0.49 & 0.65 \\
\hline 0.43 & 2.59 & 4.42 & 6.96 & 11.56 & 0.84 & 0.28 & 0.38 & 0.49 & 0.64 \\
\hline 0.44 & 2.38 & 4.03 & 6.33 & 10.47 & 0.85 & 0.27 & 0.37 & 0.48 & 0.64 \\
\hline 0.45 & 2.18 & 3.67 & 5.74 & 9.50 & 0.86 & 0.27 & 0.37 & 0.48 & 0.63 \\
\hline 0.46 & 2.00 & 3.36 & 5.22 & 8.63 & 0.87 & 0.27 & 0.36 & 0.47 & 0.62 \\
\hline 0.47 & 1.84 & 3.06 & 4.75 & 7.78 & 0.88 & 0.27 & 0.36 & 0.46 & 0.62 \\
\hline 0.48 & 1.70 & 2.80 & 4.33 & 7.06 & 0.89 & 0.26 & 0.36 & 0.46 & 0.61 \\
\hline 0.49 & 1.56 & 2.55 & 3.93 & 6.38 & 0.90 & 0.26 & 0.35 & 0.45 & 0.60 \\
\hline 0.50 & 1.44 & 2.34 & 3.57 & 5.80 & & & & & \\
\hline
\end{tabular}

Table 6: Critical values for FM-OLS \& D-OLS with one regressor for the intercept case. 


\begin{tabular}{|c|c|c|c|c|c|c|c|c|c|}
\hline$m$ & $90 \%$ & $95 \%$ & $97.5 \%$ & $99 \%$ & $m$ & $90 \%$ & $95 \%$ & $97.5 \%$ & $99 \%$ \\
\hline 0.10 & 3205.88 & 5325.61 & 8212.84 & 13425.47 & 0.51 & 4.42 & 6.85 & 9.95 & 15.33 \\
\hline 0.11 & 2338.95 & 3866.29 & 5982.86 & 9753.77 & 0.52 & 3.93 & 6.10 & 8.85 & 13.59 \\
\hline 0.12 & 1741.12 & 2875.63 & 4417.46 & 7231.43 & 0.53 & 3.51 & 5.44 & 7.88 & 12.07 \\
\hline 0.13 & 1320.81 & 2181.13 & 3363.93 & 5486.46 & 0.54 & 3.14 & 4.85 & 6.99 & 10.67 \\
\hline 0.14 & 1023.54 & 1683.19 & 2581.31 & 4222.53 & 0.55 & 2.81 & 4.32 & 6.21 & 9.50 \\
\hline 0.15 & 803.93 & 1323.84 & 2030.97 & 3293.20 & 0.56 & 2.50 & 3.83 & 5.50 & 8.43 \\
\hline 0.16 & 638.34 & 1048.89 & 1610.98 & 2613.38 & 0.57 & 2.23 & 3.42 & 4.90 & 7.44 \\
\hline 0.17 & 514.48 & 845.20 & 1296.56 & 2104.27 & 0.58 & 1.99 & 3.05 & 4.36 & 6.58 \\
\hline 0.18 & 417.80 & 687.25 & 1054.12 & 1711.22 & 0.59 & 1.78 & 2.72 & 3.88 & 5.83 \\
\hline 0.19 & 343.32 & 562.83 & 860.87 & 1399.28 & 0.60 & 1.59 & 2.43 & 3.44 & 5.15 \\
\hline 0.20 & 283.73 & 464.96 & 709.89 & 1149.95 & 0.61 & 1.42 & 2.15 & 3.05 & 4.57 \\
\hline 0.21 & 236.56 & 386.21 & 591.37 & 954.52 & 0.62 & 1.26 & 1.91 & 2.71 & 4.06 \\
\hline 0.22 & 198.19 & 323.69 & 494.83 & 800.37 & 0.63 & 1.12 & 1.69 & 2.39 & 3.56 \\
\hline 0.23 & 167.04 & 272.48 & 416.71 & 676.20 & 0.64 & 1.00 & 1.50 & 2.11 & 3.15 \\
\hline 0.24 & 141.49 & 230.35 & 351.74 & 568.17 & 0.65 & 0.89 & 1.33 & 1.87 & 2.78 \\
\hline 0.25 & 120.65 & 195.58 & 297.08 & 481.41 & 0.66 & 0.79 & 1.18 & 1.66 & 2.45 \\
\hline 0.26 & 102.92 & 166.91 & 253.84 & 409.60 & 0.67 & 0.71 & 1.04 & 1.46 & 2.15 \\
\hline 0.27 & 88.33 & 142.87 & 217.42 & 349.75 & 0.68 & 0.63 & 0.92 & 1.29 & 1.89 \\
\hline 0.28 & 76.20 & 123.56 & 186.78 & 300.96 & 0.69 & 0.56 & 0.81 & 1.13 & 1.66 \\
\hline 0.29 & 65.95 & 106.60 & 161.16 & 259.16 & 0.70 & 0.51 & 0.72 & 0.99 & 1.45 \\
\hline 0.30 & 57.22 & 92.54 & 139.93 & 223.76 & 0.71 & 0.46 & 0.64 & 0.87 & 1.26 \\
\hline 0.31 & 49.82 & 80.61 & 121.45 & 194.05 & 0.72 & 0.41 & 0.57 & 0.76 & 1.10 \\
\hline 0.32 & 43.65 & 70.07 & 105.78 & 168.11 & 0.73 & 0.37 & 0.51 & 0.67 & 0.95 \\
\hline 0.33 & 38.07 & 61.16 & 91.95 & 146.09 & 0.74 & 0.34 & 0.45 & 0.59 & 0.82 \\
\hline 0.34 & 33.36 & 53.51 & 80.53 & 128.04 & 0.75 & 0.31 & 0.41 & 0.52 & 0.72 \\
\hline 0.35 & 29.27 & 46.81 & 70.38 & 111.98 & 0.76 & 0.28 & 0.37 & 0.47 & 0.63 \\
\hline 0.36 & 25.70 & 41.15 & 61.75 & 98.46 & 0.77 & 0.26 & 0.33 & 0.42 & 0.55 \\
\hline 0.37 & 22.72 & 36.37 & 54.33 & 86.55 & 0.78 & 0.24 & 0.31 & 0.38 & 0.49 \\
\hline 0.38 & 20.04 & 32.00 & 47.60 & 75.75 & 0.79 & 0.23 & 0.28 & 0.35 & 0.44 \\
\hline 0.39 & 17.75 & 28.28 & 42.28 & 66.64 & 0.80 & 0.21 & 0.26 & 0.32 & 0.40 \\
\hline 0.40 & 15.70 & 25.03 & 37.47 & 58.94 & 0.81 & 0.20 & 0.25 & 0.30 & 0.37 \\
\hline 0.41 & 13.92 & 22.13 & 32.91 & 51.92 & 0.82 & 0.19 & 0.23 & 0.28 & 0.35 \\
\hline 0.42 & 12.37 & 19.60 & 29.07 & 45.88 & 0.83 & 0.18 & 0.22 & 0.26 & 0.33 \\
\hline 0.43 & 11.01 & 17.40 & 25.75 & 40.46 & 0.84 & 0.17 & 0.21 & 0.25 & 0.31 \\
\hline 0.44 & 9.82 & 15.47 & 22.79 & 35.75 & 0.85 & 0.16 & 0.20 & 0.24 & 0.30 \\
\hline 0.45 & 8.74 & 13.72 & 20.29 & 31.72 & 0.86 & 0.16 & 0.19 & 0.23 & 0.29 \\
\hline 0.46 & 7.77 & 12.19 & 17.92 & 28.27 & 0.87 & 0.15 & 0.19 & 0.22 & 0.28 \\
\hline 0.47 & 6.92 & 10.86 & 15.93 & 24.84 & 0.88 & 0.14 & 0.18 & 0.22 & 0.27 \\
\hline 0.48 & 6.20 & 9.68 & 14.21 & 22.00 & 0.89 & 0.14 & 0.17 & 0.21 & 0.26 \\
\hline 0.49 & 5.54 & 8.63 & 12.61 & 19.58 & 0.90 & 0.14 & 0.17 & 0.20 & 0.25 \\
\hline 0.50 & 4.95 & 7.67 & 11.22 & 17.35 & & & & & \\
\hline
\end{tabular}

Table 7: Critical values for FM-OLS \& D-OLS with one regressor for the intercept and linear trend case. 


\begin{tabular}{|c|c|c|c|c|c|c|c|c|c|}
\hline$m$ & $90 \%$ & $95 \%$ & $97.5 \%$ & $99 \%$ & $m$ & $90 \%$ & $95 \%$ & $97.5 \%$ & $99 \%$ \\
\hline 0.10 & 302.01 & 616.19 & 1123.15 & 2164.76 & 0.51 & 2.51 & 4.47 & 7.37 & 13.14 \\
\hline 0.11 & 241.96 & 493.68 & 892.29 & 1746.33 & 0.52 & 2.31 & 4.09 & 6.72 & 11.88 \\
\hline 0.12 & 196.96 & 401.15 & 727.88 & 1416.39 & 0.53 & 2.12 & 3.73 & 6.11 & 10.80 \\
\hline 0.13 & 163.30 & 331.04 & 598.38 & 1168.42 & 0.54 & 1.95 & 3.42 & 5.57 & 9.79 \\
\hline 0.14 & 136.16 & 275.69 & 499.38 & 964.93 & 0.55 & 1.79 & 3.13 & 5.07 & 8.91 \\
\hline 0.15 & 114.69 & 232.45 & 417.34 & 807.86 & 0.56 & 1.65 & 2.86 & 4.62 & 8.08 \\
\hline 0.16 & 97.31 & 196.84 & 354.39 & 684.46 & 0.57 & 1.51 & 2.61 & 4.20 & 7.29 \\
\hline 0.17 & 83.27 & 167.74 & 301.22 & 577.64 & 0.58 & 1.39 & 2.38 & 3.82 & 6.59 \\
\hline 0.18 & 71.82 & 144.46 & 258.47 & 495.75 & 0.59 & 1.27 & 2.18 & 3.47 & 5.96 \\
\hline 0.19 & 62.20 & 124.50 & 222.98 & 430.48 & 0.60 & 1.17 & 1.99 & 3.15 & 5.39 \\
\hline 0.20 & 54.42 & 108.62 & 193.99 & 371.84 & 0.61 & 1.07 & 1.81 & 2.85 & 4.90 \\
\hline 0.21 & 47.71 & 94.86 & 169.98 & 324.57 & 0.62 & 0.98 & 1.65 & 2.59 & 4.44 \\
\hline 0.22 & 41.90 & 83.56 & 149.20 & 284.74 & 0.63 & 0.90 & 1.50 & 2.36 & 4.01 \\
\hline 0.23 & 37.10 & 73.76 & 131.23 & 248.97 & 0.64 & 0.82 & 1.37 & 2.14 & 3.61 \\
\hline 0.24 & 32.97 & 65.32 & 115.76 & 220.59 & 0.65 & 0.75 & 1.25 & 1.94 & 3.25 \\
\hline 0.25 & 29.34 & 57.81 & 102.68 & 195.35 & 0.66 & 0.69 & 1.13 & 1.76 & 2.93 \\
\hline 0.26 & 26.15 & 51.51 & 91.22 & 173.74 & 0.67 & 0.63 & 1.03 & 1.59 & 2.63 \\
\hline 0.27 & 23.37 & 45.91 & 81.20 & 154.67 & 0.68 & 0.57 & 0.93 & 1.43 & 2.37 \\
\hline 0.28 & 20.96 & 41.03 & 72.56 & 137.34 & 0.69 & 0.52 & 0.84 & 1.29 & 2.11 \\
\hline 0.29 & 18.88 & 36.74 & 64.76 & 122.15 & 0.70 & 0.47 & 0.76 & 1.16 & 1.90 \\
\hline 0.30 & 17.01 & 33.02 & 57.93 & 108.59 & 0.71 & 0.43 & 0.69 & 1.04 & 1.70 \\
\hline 0.31 & 15.35 & 29.73 & 52.00 & 97.75 & 0.72 & 0.39 & 0.62 & 0.93 & 1.51 \\
\hline 0.32 & 13.90 & 26.90 & 46.73 & 87.99 & 0.73 & 0.35 & 0.56 & 0.84 & 1.35 \\
\hline 0.33 & 12.60 & 24.30 & 42.13 & 78.89 & 0.74 & 0.32 & 0.50 & 0.75 & 1.20 \\
\hline 0.34 & 11.41 & 21.97 & 38.08 & 70.78 & 0.75 & 0.29 & 0.45 & 0.67 & 1.06 \\
\hline 0.35 & 10.38 & 19.85 & 34.37 & 63.50 & 0.76 & 0.26 & 0.40 & 0.59 & 0.94 \\
\hline 0.36 & 9.44 & 18.01 & 31.16 & 57.60 & 0.77 & 0.23 & 0.36 & 0.53 & 0.83 \\
\hline 0.37 & 8.59 & 16.31 & 28.20 & 51.74 & 0.78 & 0.21 & 0.32 & 0.47 & 0.73 \\
\hline 0.38 & 7.82 & 14.79 & 25.47 & 46.81 & 0.79 & 0.19 & 0.29 & 0.41 & 0.64 \\
\hline 0.39 & 7.13 & 13.43 & 23.03 & 42.45 & 0.80 & 0.18 & 0.26 & 0.37 & 0.56 \\
\hline 0.40 & 6.51 & 12.21 & 20.90 & 38.47 & 0.81 & 0.16 & 0.23 & 0.32 & 0.49 \\
\hline 0.41 & 5.96 & 11.12 & 18.96 & 34.86 & 0.82 & 0.15 & 0.21 & 0.29 & 0.43 \\
\hline 0.42 & 5.46 & 10.14 & 17.22 & 31.48 & 0.83 & 0.14 & 0.19 & 0.25 & 0.37 \\
\hline 0.43 & 4.99 & 9.23 & 15.63 & 28.61 & 0.84 & 0.13 & 0.17 & 0.23 & 0.33 \\
\hline 0.44 & 4.57 & 8.42 & 14.21 & 25.91 & 0.85 & 0.12 & 0.16 & 0.21 & 0.29 \\
\hline 0.45 & 4.19 & 7.68 & 12.96 & 23.53 & 0.86 & 0.11 & 0.15 & 0.19 & 0.26 \\
\hline 0.46 & 3.85 & 7.00 & 11.78 & 21.32 & 0.87 & 0.11 & 0.14 & 0.18 & 0.24 \\
\hline 0.47 & 3.53 & 6.41 & 10.75 & 19.38 & 0.88 & 0.10 & 0.13 & 0.17 & 0.22 \\
\hline 0.48 & 3.24 & 5.86 & 9.79 & 17.55 & 0.89 & 0.10 & 0.13 & 0.16 & 0.21 \\
\hline 0.49 & 2.97 & 5.35 & 8.91 & 15.96 & 0.90 & 0.10 & 0.12 & 0.16 & 0.20 \\
\hline 0.50 & 2.73 & 4.89 & 8.11 & 14.45 & & & & & \\
\hline
\end{tabular}

Table 8: Critical values for IM-OLS with one regressor for the intercept case. 


\begin{tabular}{|c|c|c|c|c|c|c|c|c|c|}
\hline$m$ & $90 \%$ & $95 \%$ & $97.5 \%$ & $99 \%$ & $m$ & $90 \%$ & $95 \%$ & $97.5 \%$ & $99 \%$ \\
\hline 0.10 & 5714.91 & 10161.34 & 16644.37 & 29142.29 & 0.51 & 7.86 & 12.94 & 20.08 & 33.62 \\
\hline 0.11 & 4152.40 & 7378.48 & 12117.97 & 21252.75 & 0.52 & 7.03 & 11.55 & 17.83 & 29.76 \\
\hline 0.12 & 3089.93 & 5464.02 & 8971.08 & 15675.90 & 0.53 & 6.29 & 10.31 & 15.89 & 26.53 \\
\hline 0.13 & 2342.37 & 4146.13 & 6756.60 & 11835.05 & 0.54 & 5.62 & 9.21 & 14.14 & 23.58 \\
\hline 0.14 & 1806.26 & 3197.50 & 5215.71 & 9125.17 & 0.55 & 5.03 & 8.21 & 12.61 & 20.91 \\
\hline 0.15 & 1412.96 & 2501.02 & 4084.95 & 7165.94 & 0.56 & 4.50 & 7.33 & 11.27 & 18.47 \\
\hline 0.16 & 1120.95 & 1980.70 & 3244.39 & 5689.81 & 0.57 & 4.03 & 6.54 & 10.03 & 16.43 \\
\hline 0.17 & 902.07 & 1587.83 & 2604.16 & 4539.43 & 0.58 & 3.60 & 5.83 & 8.91 & 14.62 \\
\hline 0.18 & 734.42 & 1290.14 & 2106.33 & 3677.08 & 0.59 & 3.21 & 5.21 & 7.94 & 12.98 \\
\hline 0.19 & 602.14 & 1056.42 & 1729.21 & 2995.80 & 0.60 & 2.87 & 4.65 & 7.07 & 11.53 \\
\hline 0.20 & 497.42 & 873.46 & 1420.37 & 2473.81 & 0.61 & 2.57 & 4.15 & 6.28 & 10.22 \\
\hline 0.21 & 413.63 & 724.41 & 1179.08 & 2052.30 & 0.62 & 2.30 & 3.69 & 5.58 & 9.02 \\
\hline 0.22 & 346.15 & 604.26 & 988.04 & 1708.32 & 0.63 & 2.06 & 3.29 & 4.94 & 7.94 \\
\hline 0.23 & 291.81 & 511.02 & 832.81 & 1445.81 & 0.64 & 1.84 & 2.93 & 4.38 & 7.03 \\
\hline 0.24 & 247.93 & 432.50 & 702.19 & 1222.98 & 0.65 & 1.64 & 2.60 & 3.88 & 6.19 \\
\hline 0.25 & 210.91 & 367.02 & 596.79 & 1036.96 & 0.66 & 1.46 & 2.31 & 3.44 & 5.45 \\
\hline 0.26 & 180.55 & 314.53 & 509.13 & 880.85 & 0.67 & 1.30 & 2.05 & 3.04 & 4.80 \\
\hline 0.27 & 154.91 & 269.85 & 435.13 & 749.23 & 0.68 & 1.15 & 1.81 & 2.68 & 4.22 \\
\hline 0.28 & 133.47 & 231.82 & 373.85 & 642.20 & 0.69 & 1.02 & 1.61 & 2.36 & 3.71 \\
\hline 0.29 & 115.45 & 200.84 & 322.62 & 557.63 & 0.70 & 0.91 & 1.42 & 2.08 & 3.26 \\
\hline 0.30 & 100.24 & 173.63 & 279.36 & 481.82 & 0.71 & 0.81 & 1.25 & 1.83 & 2.85 \\
\hline 0.31 & 87.31 & 150.80 & 243.53 & 417.67 & 0.72 & 0.71 & 1.10 & 1.61 & 2.49 \\
\hline 0.32 & 76.18 & 131.32 & 211.84 & 362.84 & 0.73 & 0.63 & 0.97 & 1.41 & 2.17 \\
\hline 0.33 & 66.58 & 114.85 & 184.51 & 316.22 & 0.74 & 0.55 & 0.85 & 1.23 & 1.89 \\
\hline 0.34 & 58.44 & 100.30 & 161.13 & 276.70 & 0.75 & 0.49 & 0.75 & 1.08 & 1.64 \\
\hline 0.35 & 51.38 & 87.90 & 140.91 & 242.11 & 0.76 & 0.42 & 0.65 & 0.94 & 1.42 \\
\hline 0.36 & 45.19 & 77.32 & 123.37 & 211.84 & 0.77 & 0.37 & 0.57 & 0.81 & 1.23 \\
\hline 0.37 & 39.92 & 68.12 & 108.66 & 186.27 & 0.78 & 0.32 & 0.49 & 0.70 & 1.06 \\
\hline 0.38 & 35.23 & 60.01 & 95.72 & 164.54 & 0.79 & 0.28 & 0.43 & 0.61 & 0.91 \\
\hline 0.39 & 31.21 & 52.92 & 84.43 & 144.50 & 0.80 & 0.24 & 0.37 & 0.52 & 0.78 \\
\hline 0.40 & 27.67 & 46.93 & 74.67 & 127.68 & 0.81 & 0.21 & 0.32 & 0.45 & 0.66 \\
\hline 0.41 & 24.54 & 41.62 & 65.99 & 112.02 & 0.82 & 0.18 & 0.27 & 0.38 & 0.56 \\
\hline 0.42 & 21.81 & 36.86 & 58.36 & 99.02 & 0.83 & 0.16 & 0.23 & 0.32 & 0.47 \\
\hline 0.43 & 19.37 & 32.76 & 51.63 & 87.88 & 0.84 & 0.14 & 0.20 & 0.27 & 0.39 \\
\hline 0.44 & 17.26 & 29.06 & 45.94 & 78.07 & 0.85 & 0.12 & 0.17 & 0.23 & 0.33 \\
\hline 0.45 & 15.39 & 25.83 & 40.86 & 69.45 & 0.86 & 0.11 & 0.15 & 0.19 & 0.27 \\
\hline 0.46 & 13.71 & 23.00 & 36.30 & 61.27 & 0.87 & 0.10 & 0.13 & 0.17 & 0.23 \\
\hline 0.47 & 12.26 & 20.53 & 32.13 & 54.29 & 0.88 & 0.09 & 0.12 & 0.15 & 0.19 \\
\hline 0.48 & 10.97 & 18.24 & 28.52 & 47.92 & 0.89 & 0.09 & 0.11 & 0.13 & 0.17 \\
\hline 0.49 & 9.82 & 16.25 & 25.36 & 42.53 & 0.90 & 0.08 & 0.10 & 0.12 & 0.15 \\
\hline 0.50 & 8.77 & 14.50 & 22.56 & 37.91 & & & & & \\
\hline
\end{tabular}

Table 9: Critical values for IM-OLS with one regressor for the intercept and linear case. 


\begin{tabular}{|c|c|c|c|c|c|c|c|c|c|}
\hline$m$ & $90 \%$ & $95 \%$ & $97.5 \%$ & $99 \%$ & $m$ & $90 \%$ & $95 \%$ & $97.5 \%$ & $99 \%$ \\
\hline 0.10 & 426.04 & 748.19 & 1192.55 & 1993.76 & 0.51 & 2.56 & 4.20 & 6.32 & 9.98 \\
\hline 0.11 & 339.66 & 598.44 & 950.03 & 1583.82 & 0.52 & 2.32 & 3.80 & 5.72 & 8.96 \\
\hline 0.12 & 275.97 & 485.69 & 771.64 & 1283.15 & 0.53 & 2.11 & 3.45 & 5.17 & 8.10 \\
\hline 0.13 & 226.07 & 398.20 & 630.19 & 1048.84 & 0.54 & 1.91 & 3.11 & 4.67 & 7.29 \\
\hline 0.14 & 188.00 & 330.85 & 526.34 & 869.44 & 0.55 & 1.73 & 2.82 & 4.21 & 6.59 \\
\hline 0.15 & 157.66 & 276.31 & 438.86 & 729.21 & 0.56 & 1.57 & 2.54 & 3.77 & 5.88 \\
\hline 0.16 & 133.67 & 234.35 & 372.69 & 618.66 & 0.57 & 1.42 & 2.29 & 3.40 & 5.28 \\
\hline 0.17 & 113.74 & 199.44 & 315.97 & 523.14 & 0.58 & 1.29 & 2.06 & 3.07 & 4.74 \\
\hline 0.18 & 97.51 & 170.79 & 269.60 & 447.51 & 0.59 & 1.16 & 1.86 & 2.76 & 4.24 \\
\hline 0.19 & 84.09 & 147.37 & 233.22 & 385.43 & 0.60 & 1.05 & 1.68 & 2.47 & 3.79 \\
\hline 0.20 & 73.26 & 127.98 & 201.10 & 333.61 & 0.61 & 0.95 & 1.51 & 2.21 & 3.40 \\
\hline 0.21 & 63.97 & 111.20 & 174.87 & 289.85 & 0.62 & 0.86 & 1.35 & 1.98 & 3.03 \\
\hline 0.22 & 55.65 & 96.98 & 152.83 & 250.75 & 0.63 & 0.78 & 1.22 & 1.77 & 2.71 \\
\hline 0.23 & 49.04 & 85.23 & 133.95 & 218.94 & 0.64 & 0.71 & 1.09 & 1.58 & 2.40 \\
\hline 0.24 & 43.18 & 75.03 & 117.57 & 191.89 & 0.65 & 0.64 & 0.98 & 1.41 & 2.14 \\
\hline 0.25 & 38.03 & 66.26 & 103.91 & 169.35 & 0.66 & 0.58 & 0.88 & 1.26 & 1.90 \\
\hline 0.26 & 33.84 & 58.48 & 91.78 & 150.18 & 0.67 & 0.53 & 0.79 & 1.12 & 1.68 \\
\hline 0.27 & 30.11 & 51.95 & 81.22 & 132.23 & 0.68 & 0.48 & 0.71 & 1.00 & 1.49 \\
\hline 0.28 & 26.79 & 46.32 & 72.12 & 118.02 & 0.69 & 0.44 & 0.64 & 0.90 & 1.31 \\
\hline 0.29 & 23.94 & 41.30 & 64.13 & 104.68 & 0.70 & 0.41 & 0.59 & 0.80 & 1.16 \\
\hline 0.30 & 21.38 & 36.85 & 57.31 & 93.49 & 0.71 & 0.37 & 0.53 & 0.72 & 1.03 \\
\hline 0.31 & 19.12 & 32.90 & 51.32 & 83.26 & 0.72 & 0.35 & 0.49 & 0.65 & 0.92 \\
\hline 0.32 & 17.18 & 29.51 & 46.10 & 74.66 & 0.73 & 0.32 & 0.45 & 0.59 & 0.83 \\
\hline 0.33 & 15.44 & 26.52 & 41.18 & 66.33 & 0.74 & 0.30 & 0.41 & 0.54 & 0.75 \\
\hline 0.34 & 13.91 & 23.75 & 36.73 & 59.41 & 0.75 & 0.28 & 0.38 & 0.50 & 0.68 \\
\hline 0.35 & 12.50 & 21.24 & 32.98 & 53.07 & 0.76 & 0.26 & 0.36 & 0.46 & 0.63 \\
\hline 0.36 & 11.28 & 19.16 & 29.70 & 47.62 & 0.77 & 0.25 & 0.34 & 0.44 & 0.58 \\
\hline 0.37 & 10.19 & 17.24 & 26.58 & 42.79 & 0.78 & 0.24 & 0.32 & 0.41 & 0.55 \\
\hline 0.38 & 9.21 & 15.56 & 23.96 & 38.35 & 0.79 & 0.23 & 0.30 & 0.39 & 0.52 \\
\hline 0.39 & 8.33 & 14.06 & 21.64 & 34.66 & 0.80 & 0.22 & 0.29 & 0.38 & 0.50 \\
\hline 0.40 & 7.53 & 12.68 & 19.51 & 31.22 & 0.81 & 0.21 & 0.28 & 0.36 & 0.49 \\
\hline 0.41 & 6.82 & 11.45 & 17.59 & 28.11 & 0.82 & 0.21 & 0.27 & 0.35 & 0.47 \\
\hline 0.42 & 6.18 & 10.36 & 15.91 & 25.44 & 0.83 & 0.20 & 0.27 & 0.35 & 0.47 \\
\hline 0.43 & 5.59 & 9.40 & 14.37 & 22.94 & 0.84 & 0.20 & 0.26 & 0.34 & 0.46 \\
\hline 0.44 & 5.08 & 8.50 & 12.94 & 20.65 & 0.85 & 0.19 & 0.26 & 0.34 & 0.45 \\
\hline 0.45 & 4.61 & 7.68 & 11.70 & 18.52 & 0.86 & 0.19 & 0.26 & 0.33 & 0.45 \\
\hline 0.46 & 4.17 & 6.96 & 10.56 & 16.79 & 0.87 & 0.19 & 0.25 & 0.33 & 0.44 \\
\hline 0.47 & 3.78 & 6.28 & 9.57 & 15.10 & 0.88 & 0.19 & 0.25 & 0.32 & 0.44 \\
\hline 0.48 & 3.43 & 5.69 & 8.62 & 13.55 & 0.89 & 0.18 & 0.25 & 0.32 & 0.43 \\
\hline 0.49 & 3.11 & 5.13 & 7.77 & 12.25 & 0.90 & 0.18 & 0.24 & 0.32 & 0.43 \\
\hline 0.50 & 2.82 & 4.64 & 6.99 & 11.05 & & & & & \\
\hline
\end{tabular}

Table 10: Critical values for FM-OLS \& D-OLS with two regressors for the intercept case. 


\begin{tabular}{|c|c|c|c|c|c|c|c|c|c|}
\hline$m$ & $90 \%$ & $95 \%$ & $97.5 \%$ & $99 \%$ & $m$ & $90 \%$ & $95 \%$ & $97.5 \%$ & $99 \%$ \\
\hline 0.10 & 853.59 & 1594.54 & 2671.27 & 4698.76 & 0.51 & 5.15 & 8.90 & 14.12 & 23.97 \\
\hline 0.11 & 681.29 & 1268.17 & 2124.67 & 3762.92 & 0.52 & 4.67 & 8.07 & 12.79 & 21.56 \\
\hline 0.12 & 550.84 & 1026.96 & 1712.63 & 3043.26 & 0.53 & 4.23 & 7.31 & 11.58 & 19.40 \\
\hline 0.13 & 452.09 & 839.08 & 1410.65 & 2513.50 & 0.54 & 3.84 & 6.63 & 10.46 & 17.46 \\
\hline 0.14 & 375.72 & 696.55 & 1167.20 & 2070.47 & 0.55 & 3.49 & 6.00 & 9.45 & 15.71 \\
\hline 0.15 & 314.58 & 584.99 & 979.70 & 1741.06 & 0.56 & 3.16 & 5.42 & 8.53 & 14.20 \\
\hline 0.16 & 266.22 & 494.56 & 824.15 & 1460.65 & 0.57 & 2.87 & 4.90 & 7.69 & 12.79 \\
\hline 0.17 & 226.88 & 420.42 & 701.09 & 1243.68 & 0.58 & 2.60 & 4.42 & 6.92 & 11.51 \\
\hline 0.18 & 194.33 & 359.30 & 596.49 & 1058.81 & 0.59 & 2.35 & 3.99 & 6.23 & 10.31 \\
\hline 0.19 & 167.59 & 309.51 & 514.22 & 906.80 & 0.60 & 2.13 & 3.59 & 5.59 & 9.26 \\
\hline 0.20 & 145.33 & 267.50 & 445.66 & 785.03 & 0.61 & 1.93 & 3.23 & 5.03 & 8.31 \\
\hline 0.21 & 126.91 & 233.60 & 387.75 & 678.19 & 0.62 & 1.74 & 2.91 & 4.50 & 7.43 \\
\hline 0.22 & 111.01 & 203.38 & 337.77 & 593.70 & 0.63 & 1.57 & 2.63 & 4.04 & 6.63 \\
\hline 0.23 & 97.74 & 178.16 & 296.22 & 520.60 & 0.64 & 1.42 & 2.36 & 3.61 & 5.92 \\
\hline 0.24 & 86.07 & 157.75 & 260.18 & 457.41 & 0.65 & 1.28 & 2.12 & 3.24 & 5.28 \\
\hline 0.25 & 76.12 & 139.14 & 229.90 & 401.84 & 0.66 & 1.15 & 1.90 & 2.88 & 4.69 \\
\hline 0.26 & 67.51 & 123.07 & 202.49 & 355.06 & 0.67 & 1.03 & 1.70 & 2.58 & 4.16 \\
\hline 0.27 & 59.99 & 109.05 & 179.55 & 315.19 & 0.68 & 0.93 & 1.52 & 2.30 & 3.68 \\
\hline 0.28 & 53.50 & 97.40 & 159.68 & 279.33 & 0.69 & 0.83 & 1.36 & 2.05 & 3.27 \\
\hline 0.29 & 47.71 & 86.71 & 142.13 & 248.23 & 0.70 & 0.74 & 1.21 & 1.82 & 2.90 \\
\hline 0.30 & 42.69 & 77.38 & 126.72 & 221.69 & 0.71 & 0.66 & 1.08 & 1.62 & 2.56 \\
\hline 0.31 & 38.12 & 69.12 & 113.58 & 198.01 & 0.72 & 0.59 & 0.96 & 1.43 & 2.26 \\
\hline 0.32 & 34.20 & 61.83 & 101.68 & 177.45 & 0.73 & 0.52 & 0.84 & 1.26 & 1.99 \\
\hline 0.33 & 30.76 & 55.63 & 91.50 & 158.15 & 0.74 & 0.47 & 0.75 & 1.11 & 1.75 \\
\hline 0.34 & 27.72 & 49.87 & 81.77 & 141.88 & 0.75 & 0.41 & 0.66 & 0.98 & 1.53 \\
\hline 0.35 & 24.95 & 44.75 & 73.24 & 127.65 & 0.76 & 0.36 & 0.58 & 0.85 & 1.33 \\
\hline 0.36 & 22.50 & 40.24 & 65.73 & 114.48 & 0.77 & 0.32 & 0.51 & 0.74 & 1.16 \\
\hline 0.37 & 20.29 & 36.32 & 58.99 & 102.50 & 0.78 & 0.28 & 0.44 & 0.65 & 1.00 \\
\hline 0.38 & 18.35 & 32.81 & 53.10 & 91.94 & 0.79 & 0.24 & 0.38 & 0.56 & 0.87 \\
\hline 0.39 & 16.57 & 29.56 & 47.95 & 82.62 & 0.80 & 0.21 & 0.33 & 0.48 & 0.75 \\
\hline 0.40 & 14.99 & 26.64 & 43.15 & 74.36 & 0.81 & 0.18 & 0.29 & 0.42 & 0.64 \\
\hline 0.41 & 13.59 & 24.07 & 38.81 & 66.55 & 0.82 & 0.16 & 0.24 & 0.36 & 0.54 \\
\hline 0.42 & 12.31 & 21.80 & 35.10 & 59.80 & 0.83 & 0.13 & 0.21 & 0.30 & 0.46 \\
\hline 0.43 & 11.17 & 19.75 & 31.71 & 53.74 & 0.84 & 0.11 & 0.18 & 0.25 & 0.38 \\
\hline 0.44 & 10.13 & 17.90 & 28.62 & 48.55 & 0.85 & 0.10 & 0.15 & 0.21 & 0.32 \\
\hline 0.45 & 9.18 & 16.19 & 25.84 & 43.82 & 0.86 & 0.09 & 0.13 & 0.18 & 0.26 \\
\hline 0.46 & 8.33 & 14.65 & 23.38 & 39.55 & 0.87 & 0.08 & 0.11 & 0.15 & 0.22 \\
\hline 0.47 & 7.55 & 13.24 & 21.13 & 35.92 & 0.88 & 0.07 & 0.09 & 0.12 & 0.18 \\
\hline 0.48 & 6.84 & 11.97 & 19.12 & 32.30 & 0.89 & 0.07 & 0.08 & 0.11 & 0.15 \\
\hline 0.49 & 6.21 & 10.84 & 17.24 & 29.29 & 0.90 & 0.06 & 0.08 & 0.10 & 0.13 \\
\hline 0.50 & 5.65 & 9.84 & 15.62 & 26.56 & & & & & \\
\hline
\end{tabular}

Table 11: Critical values for IM-OLS with two regressors for the intercept case. 


\begin{tabular}{|c|c|c|c|c|c|c|c|c|c|}
\hline$m$ & $90 \%$ & $95 \%$ & $97.5 \%$ & $99 \%$ & $m$ & $90 \%$ & $95 \%$ & $97.5 \%$ & $99 \%$ \\
\hline 0.10 & 6200.37 & 10193.95 & 15326.19 & 24073.55 & 0.51 & 7.23 & 11.48 & 16.82 & 25.63 \\
\hline 0.11 & 4509.27 & 7377.93 & 11123.03 & 17501.96 & 0.52 & 6.43 & 10.18 & 14.93 & 22.63 \\
\hline 0.12 & 3352.96 & 5490.25 & 8244.13 & 12962.80 & 0.53 & 5.72 & 9.04 & 13.18 & 19.96 \\
\hline 0.13 & 2538.73 & 4164.23 & 6236.31 & 9813.63 & 0.54 & 5.08 & 8.00 & 11.67 & 17.77 \\
\hline 0.14 & 1955.13 & 3206.43 & 4839.43 & 7570.52 & 0.55 & 4.50 & 7.10 & 10.31 & 15.64 \\
\hline 0.15 & 1532.08 & 2516.54 & 3784.19 & 5980.17 & 0.56 & 3.98 & 6.25 & 9.09 & 13.82 \\
\hline 0.16 & 1216.30 & 1996.07 & 3005.41 & 4722.84 & 0.57 & 3.54 & 5.53 & 8.03 & 12.20 \\
\hline 0.17 & 973.37 & 1601.75 & 2405.88 & 3775.01 & 0.58 & 3.14 & 4.90 & 7.09 & 10.65 \\
\hline 0.18 & 789.56 & 1294.82 & 1951.46 & 3066.88 & 0.59 & 2.79 & 4.33 & 6.25 & 9.40 \\
\hline 0.19 & 644.63 & 1059.77 & 1591.94 & 2503.19 & 0.60 & 2.47 & 3.83 & 5.50 & 8.28 \\
\hline 0.20 & 532.42 & 872.15 & 1310.34 & 2070.55 & 0.61 & 2.19 & 3.38 & 4.87 & 7.27 \\
\hline 0.21 & 441.97 & 723.55 & 1086.14 & 1707.82 & 0.62 & 1.93 & 2.99 & 4.31 & 6.38 \\
\hline 0.22 & 370.47 & 604.21 & 904.88 & 1418.19 & 0.63 & 1.70 & 2.64 & 3.79 & 5.62 \\
\hline 0.23 & 310.95 & 506.52 & 759.99 & 1188.72 & 0.64 & 1.50 & 2.32 & 3.31 & 4.94 \\
\hline 0.24 & 262.06 & 426.60 & 641.85 & 1004.59 & 0.65 & 1.32 & 2.03 & 2.91 & 4.31 \\
\hline 0.25 & 222.84 & 363.35 & 543.98 & 850.11 & 0.66 & 1.16 & 1.79 & 2.55 & 3.78 \\
\hline 0.26 & 190.30 & 310.19 & 462.77 & 727.46 & 0.67 & 1.02 & 1.57 & 2.23 & 3.29 \\
\hline 0.27 & 162.76 & 266.04 & 397.85 & 620.09 & 0.68 & 0.89 & 1.37 & 1.95 & 2.88 \\
\hline 0.28 & 140.04 & 228.50 & 342.80 & 534.01 & 0.69 & 0.78 & 1.19 & 1.69 & 2.49 \\
\hline 0.29 & 120.84 & 196.51 & 294.37 & 459.92 & 0.70 & 0.68 & 1.04 & 1.47 & 2.16 \\
\hline 0.30 & 104.04 & 169.63 & 254.19 & 395.40 & 0.71 & 0.60 & 0.91 & 1.28 & 1.88 \\
\hline 0.31 & 90.53 & 147.29 & 219.51 & 342.83 & 0.72 & 0.52 & 0.79 & 1.11 & 1.62 \\
\hline 0.32 & 78.57 & 127.87 & 191.05 & 297.45 & 0.73 & 0.46 & 0.68 & 0.96 & 1.39 \\
\hline 0.33 & 68.31 & 111.24 & 165.77 & 258.54 & 0.74 & 0.40 & 0.59 & 0.82 & 1.19 \\
\hline 0.34 & 59.54 & 96.83 & 144.76 & 223.95 & 0.75 & 0.35 & 0.51 & 0.70 & 1.02 \\
\hline 0.35 & 52.07 & 84.63 & 126.42 & 196.34 & 0.76 & 0.31 & 0.44 & 0.60 & 0.87 \\
\hline 0.36 & 45.79 & 74.08 & 110.23 & 172.21 & 0.77 & 0.28 & 0.38 & 0.52 & 0.74 \\
\hline 0.37 & 40.09 & 64.87 & 96.52 & 150.31 & 0.78 & 0.25 & 0.34 & 0.44 & 0.62 \\
\hline 0.38 & 35.28 & 56.91 & 84.69 & 132.06 & 0.79 & 0.22 & 0.30 & 0.38 & 0.53 \\
\hline 0.39 & 31.04 & 50.03 & 74.31 & 115.74 & 0.80 & 0.20 & 0.26 & 0.33 & 0.45 \\
\hline 0.40 & 27.43 & 44.13 & 65.53 & 101.57 & 0.81 & 0.19 & 0.24 & 0.30 & 0.39 \\
\hline 0.41 & 24.18 & 38.93 & 57.60 & 88.93 & 0.82 & 0.17 & 0.22 & 0.27 & 0.34 \\
\hline 0.42 & 21.40 & 34.31 & 50.68 & 78.33 & 0.83 & 0.16 & 0.20 & 0.24 & 0.31 \\
\hline 0.43 & 18.90 & 30.32 & 44.85 & 69.06 & 0.84 & 0.15 & 0.18 & 0.22 & 0.28 \\
\hline 0.44 & 16.76 & 26.82 & 39.45 & 60.89 & 0.85 & 0.14 & 0.17 & 0.21 & 0.26 \\
\hline 0.45 & 14.84 & 23.78 & 34.92 & 53.38 & 0.86 & 0.13 & 0.16 & 0.20 & 0.24 \\
\hline 0.46 & 13.18 & 21.00 & 30.96 & 47.39 & 0.87 & 0.13 & 0.16 & 0.19 & 0.23 \\
\hline 0.47 & 11.70 & 18.55 & 27.31 & 41.88 & 0.88 & 0.12 & 0.15 & 0.18 & 0.22 \\
\hline 0.48 & 10.35 & 16.46 & 24.17 & 37.06 & 0.89 & 0.12 & 0.14 & 0.17 & 0.22 \\
\hline 0.49 & 9.19 & 14.58 & 21.44 & 32.82 & 0.90 & 0.11 & 0.14 & 0.17 & 0.21 \\
\hline 0.50 & 8.16 & 12.95 & 18.98 & 28.96 & & & & & \\
\hline
\end{tabular}

Table 12: Critical values for FM-OLS \& D-OLS with two regressors for the intercept and linear trend case. 


\begin{tabular}{|c|c|c|c|c|c|c|c|c|c|}
\hline$m$ & $90 \%$ & $95 \%$ & $97.5 \%$ & $99 \%$ & $m$ & $90 \%$ & $95 \%$ & $97.5 \%$ & $99 \%$ \\
\hline 0.10 & 12039.65 & 20715.05 & 32501.01 & 53971.50 & 0.51 & 13.88 & 23.08 & 35.52 & 57.72 \\
\hline 0.11 & 8689.67 & 14895.60 & 23578.76 & 39302.34 & 0.52 & 12.35 & 20.56 & 31.50 & 50.87 \\
\hline 0.12 & 6432.55 & 11042.93 & 17417.93 & 29048.79 & 0.53 & 10.99 & 18.24 & 27.96 & 44.98 \\
\hline 0.13 & 4872.51 & 8398.59 & 13214.74 & 21965.82 & 0.54 & 9.77 & 16.19 & 24.80 & 39.81 \\
\hline 0.14 & 3767.09 & 6463.54 & 10161.91 & 16946.37 & 0.55 & 8.67 & 14.38 & 21.91 & 35.08 \\
\hline 0.15 & 2948.46 & 5077.83 & 7989.20 & 13197.20 & 0.56 & 7.71 & 12.75 & 19.38 & 31.01 \\
\hline 0.16 & 2339.27 & 3996.26 & 6318.34 & 10473.81 & 0.57 & 6.84 & 11.30 & 17.14 & 27.41 \\
\hline 0.17 & 1867.97 & 3211.80 & 5044.28 & 8400.40 & 0.58 & 6.08 & 9.99 & 15.19 & 24.19 \\
\hline 0.18 & 1512.38 & 2607.53 & 4089.88 & 6810.43 & 0.59 & 5.39 & 8.83 & 13.39 & 21.23 \\
\hline 0.19 & 1238.05 & 2129.85 & 3346.91 & 5552.07 & 0.60 & 4.78 & 7.81 & 11.82 & 18.74 \\
\hline 0.20 & 1018.04 & 1755.18 & 2769.11 & 4583.95 & 0.61 & 4.24 & 6.91 & 10.44 & 16.48 \\
\hline 0.21 & 842.95 & 1450.13 & 2290.85 & 3803.40 & 0.62 & 3.76 & 6.11 & 9.18 & 14.55 \\
\hline 0.22 & 705.29 & 1207.90 & 1911.03 & 3150.13 & 0.63 & 3.33 & 5.40 & 8.10 & 12.82 \\
\hline 0.23 & 592.05 & 1014.74 & 1595.03 & 2647.62 & 0.64 & 2.95 & 4.76 & 7.13 & 11.25 \\
\hline 0.24 & 499.12 & 855.29 & 1346.41 & 2235.18 & 0.65 & 2.60 & 4.20 & 6.28 & 9.89 \\
\hline 0.25 & 423.64 & 725.12 & 1139.81 & 1891.74 & 0.66 & 2.30 & 3.71 & 5.52 & 8.64 \\
\hline 0.26 & 360.94 & 8.54 & 970.63 & 1610.13 & 0.67 & 2.03 & 3.26 & 4.84 & 7.56 \\
\hline 0.27 & 310.03 & 529.29 & 832.41 & 1374.49 & 0.68 & 1.78 & 2.86 & 4.24 & 6.61 \\
\hline 0.28 & 266.49 & 456.52 & 714.64 & 1183.35 & 0.69 & 1.57 & 2.50 & 3.69 & 5.75 \\
\hline 0.29 & 229.75 & 393.51 & 615.86 & 1020.97 & 0.70 & 1.38 & 2.18 & 3.23 & 4.98 \\
\hline 0.30 & 198.60 & 339.93 & 531.44 & 881.60 & 0.71 & 1.20 & 1.91 & 2.81 & 4.33 \\
\hline 0.31 & 172.30 & 294.72 & 461.25 & 766.06 & 0.72 & 1.05 & 1.66 & 2.44 & 3.76 \\
\hline 0.32 & 149.88 & 6.24 & 401.43 & 666.86 & 0.73 & 0.92 & 1.45 & 2.12 & 3.26 \\
\hline 0.33 & 130.78 & 223.30 & 348.47 & 575.94 & 0.74 & 0.80 & 1.26 & 1.83 & 2.81 \\
\hline 0.34 & 113.98 & 4.71 & 303.25 & 500.24 & 0.75 & 0.69 & 1.09 & 1.58 & 2.42 \\
\hline 0.35 & 99.63 & 9.55 & 265.96 & 438.76 & 0.76 & 0.60 & 0.94 & 1.36 & 2.07 \\
\hline 0.36 & 87.21 & 148.69 & 233.14 & 382.53 & 0.77 & 0.52 & 0.81 & 1.16 & 1.77 \\
\hline 0.37 & 60 & 0.40 & 203.78 & 334.97 & 0.7 & 0.44 & 0.69 & 1.00 & 1.51 \\
\hline 0.38 & 67.42 & 114.24 & 178.34 & 293.07 & 0.79 & 0.38 & 0.59 & 0.85 & 1.28 \\
\hline 0.39 & 59.28 & 100.41 & 156.28 & 257.33 & 0.80 & 0.32 & 0.50 & 0.72 & 1.08 \\
\hline 0.40 & 52.23 & 88.33 & 137.28 & 226.35 & 0.81 & 0.27 & 0.42 & 0.61 & 0.90 \\
\hline 0.41 & 46.15 & 78.10 & 120.94 & 198.71 & 0.8 & 0.23 & 0.36 & 0.51 & 0.76 \\
\hline 0.42 & 40.79 & 68.71 & 106.99 & 174.90 & 0.83 & 0.19 & 0.30 & 0.42 & 0.62 \\
\hline 0.43 & 36.16 & 60.82 & 94.12 & 153.71 & 0.84 & 0.16 & 0.25 & 0.35 & 0.52 \\
\hline 0.44 & 32.04 & 53.95 & 83.05 & 135.36 & 0.8 & 0.13 & 0.20 & 0.29 & 0.42 \\
\hline 0.45 & 28.43 & 47.71 & 73.44 & 119.38 & 0.86 & 0.11 & 0.16 & 0.23 & 0.34 \\
\hline 0.46 & 25.17 & 42.29 & 65.08 & 105.37 & 0.87 & 0.09 & 0.13 & 0.19 & 0.28 \\
\hline 0.47 & 22.32 & 37.39 & 57.48 & 93.35 & 0.8 & 0.08 & 0.11 & 0.15 & 0.22 \\
\hline 0.48 & 19.79 & 33.08 & 50.99 & 82.72 & 0.89 & 0.07 & 0.09 & 0.12 & 0.17 \\
\hline 0.49 & 17.52 & 29.36 & 45.14 & 73.75 & 0.90 & 0.06 & 0.08 & 0.10 & 0.13 \\
\hline 0.50 & 15.57 & 26.03 & 40.03 & 65.03 & & & & & \\
\hline
\end{tabular}

Table 13: Critical values for IM-OLS with two regressors for the intercept and linear trend case. 


